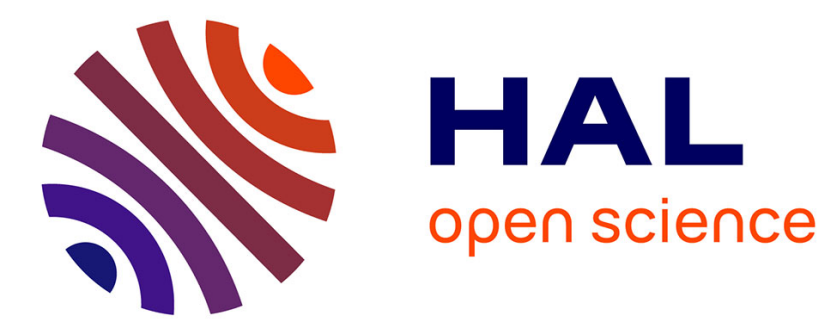

\title{
When the 3D magnetic Laplacian meets a curved edge in the semiclassical limit
}

Nicolas Popoff, Nicolas Raymond

\section{To cite this version:}

Nicolas Popoff, Nicolas Raymond. When the 3D magnetic Laplacian meets a curved edge in the semiclassical limit. SIAM Journal on Mathematical Analysis, 2013, 45 (4), pp.2354-2395. $10.1137 / 130906003$. hal-00746862v2

\section{HAL Id: hal-00746862 \\ https://hal.science/hal-00746862v2}

Submitted on 11 Jun 2013

HAL is a multi-disciplinary open access archive for the deposit and dissemination of scientific research documents, whether they are published or not. The documents may come from teaching and research institutions in France or abroad, or from public or private research centers.
L'archive ouverte pluridisciplinaire HAL, est destinée au dépôt et à la diffusion de documents scientifiques de niveau recherche, publiés ou non, émanant des établissements d'enseignement et de recherche français ou étrangers, des laboratoires publics ou privés. 


\title{
WHEN THE 3D MAGNETIC LAPLACIAN MEETS A CURVED EDGE IN THE SEMICLASSICAL LIMIT
}

\author{
NICOLAS POPOFF AND NICOLAS RAYMOND
}

\begin{abstract}
We study the magnetic Laplacian in the case when the Neumann boundary contains an edge. We provide complete asymptotic expansions in powers of $h^{1 / 4}$ of the low lying eigenpairs in the semiclassical limit $h \rightarrow 0$. In order to get our main result we establish a general method based on a normal form procedure, microlocal arguments, the Feshbach-Grushin reduction and the Born-Oppenheimer approximation.
\end{abstract}

Keywords: spectral theory, magnetic Schrödinger operator, semiclassical analysis, non smooth boundary, microlocalization, normal form.

\section{INTRODUCTION}

Let $\Omega$ be an open bounded and simply connected subset of $\mathbb{R}^{3}$ and $\left(x_{j}\right)$ be the cartesian coordinates. This paper is devoted to the spectral analysis of the Neumann realization on $\Omega$ of the magnetic Laplacian:

$$
\mathcal{L}_{h}=(-i h \nabla+\mathbf{A})^{2},
$$

where

$$
\mathbf{A}=\left(-x_{2}, 0,0\right) .
$$

When $\Omega$ is bounded and convex (cf. [18]), the domain of $\mathcal{L}_{h}$ is

$$
\operatorname{Dom}\left(\mathcal{L}_{h}\right):=\left\{u \in H^{2}(\Omega),(-i h \nabla+\mathbf{A}) u \cdot \mathbf{n}=0 \text { on } \partial \Omega\right\}
$$

where $\mathbf{n}$ is the exterior normal of the boundary. The associated quadratic form is defined for $u \in \operatorname{Dom}\left(\mathcal{Q}_{h}\right)=H^{1}(\Omega)$ :

$$
\mathcal{Q}_{h}(u):=\int_{\Omega}\left|\left(D_{x_{1}}-x_{2}\right) u\right|^{2}+\left|D_{x_{2}} u\right|^{2}+\left|D_{x_{3}} u\right|^{2} d \mathbf{x}
$$

with $D_{x_{j}}:=-i \partial_{x_{j}}$. The operator $\mathcal{L}_{h}$ has compact resolvent. By gauge invariance and since $\Omega$ is assumed to be simply connected, we know that the spectrum of $\mathcal{L}_{h}$ only depends on the magnetic field $\boldsymbol{\beta}=\nabla \times \mathbf{A}$ which is here constant: $\boldsymbol{\beta}=(0,0,1)$.

Notation 1.1. We will denote by $\mathfrak{S}\left(\mathcal{L}_{h}\right)$ the spectrum of $\mathcal{L}_{h}$ and by $\lambda_{n}(h)$ the $n$-th eigenvalue of $\mathcal{L}_{h}$.

We are interested in asymptotic expansions of $\lambda_{n}(h)$ in the semiclassical limit $h \rightarrow 0$. Our results strongly depend on the geometrical assumptions on $\Omega$ and we deal with a case when $\partial \Omega$ is not smooth, namely the boundary contains an edge $E$. We will describe the shape of $\Omega$ in Section 1.2, but we can already think to it as a lens (see Figure 1).

Date: June 3, 2013. 
1.1. Motivation and state of the art. The magnetic Laplacian has been extensively studied in the last decade in relation with the mathematical study of superconductivity. In particular, it is proved that the third critical field $H_{C_{3}}$ of the Ginzburg-Landau functional is related to the lowest eigenvalue of the magnetic Laplacian (see the papers of Lu and Pan [28, 29] and also the book of Fournais and Helffer [17]). Until now the case when the boundary of $\Omega$ is smooth was the most investigated situation. Let us describe the nature of the known results.

- Smooth domains. In 2D the constant magnetic field case is treated when $\Omega$ is a disk in $[2,3,12]$ and generalized to smooth and bounded domains by Helffer and Morame in [23] where it is proved that:

$$
\lambda_{1}(h) \sim \Theta_{0} h-C_{1} \kappa_{\max } h^{3 / 2}+o\left(h^{3 / 2}\right),
$$

where $\kappa_{\max }$ is the maximal curvature of the boundary and $\Theta_{0}$ and $C_{1}$ are constants related to the 1D de Gennes operator (see $[4,29,23,8,6]$ ). Let us briefly recall the definition of this important operator depending of the parameter $\zeta \in \mathbb{R}$ :

$$
D_{t}^{2}+(t-\zeta)^{2}, \quad t>0
$$

with Neumann boundary condition at $t=0$. Denoting by $\mu^{\mathrm{dG}}(\zeta)$ its lowest eigenvalue, we have: $\Theta_{0}:=\min _{\zeta \in \mathbb{R}} \mu^{\mathrm{dG}}(\zeta)$.

Moreover in most of the papers the authors are only concerned by the first terms of the asymptotic expansion of $\lambda_{1}(h)$. In the case of smooth domains the complete asymptotic expansion of all the eigenvalues is done by Fournais and Helffer in [16]. For the case with variable magnetic field, we refer to $[28,34]$ for the first terms of the lowest eigenvalue and to [36] for a complete expansion.

In 3D the constant magnetic field case is treated by Helffer and Morame in [25] under generic assumptions on the (smooth) boundary of $\Omega$. The authors provide a two terms expansion of $\lambda_{1}(h)$ and they prove that the associated eigenfunction concentrates on the curve where the magnetic field is tangent to the boundary of $\Omega$. More precisely, when $h$ goes to 0 , they prove the following:

$$
\lambda_{1}(h) \sim \Theta_{0} h+C(\Omega) h^{4 / 3}+o\left(h^{4 / 3}\right) .
$$

The case with variable magnetic field is analyzed in [35, 37].

- Non smooth domains. In 2D, the analysis on infinite sectors done in [5] provides a one term asymptotic expansion for $\lambda_{1}(h)$ when $\Omega$ is a curvilinear polygon. When the magnetic field is constant (and equals 1 ) and when the opening angle $\alpha_{1}$ of the smallest vertice is small enough, this expansion is in the form:

$$
\lambda_{1}(h) \sim \mu\left(\alpha_{1}\right) h+o(h)
$$

where $\mu\left(\alpha_{1}\right)$ is the lowest eigenvalue of the magnetic Laplacian (with constant magnetic field of intensity 1) on a sector of angle $\alpha_{1}$. This result is improved in [7] where the asymptotic expansion of the $n$-th eigenvalue is provided. In particular, it is observed that the splitting of the eigenvalues comes at the first order.

In [32], the case with constant magnetic field when $\Omega$ is a cuboid is addressed. In this case Pan provides the first term of the asymptotics for some special orientations of the magnetic 
field and he proves that the eigenfunctions concentrate near corners when the magnetic field is tangent to a face and not to an edge.

The 2D results obtained in $[5,7]$ can be generalized to dimension 3 in many ways (see [33]). The two extreme cases which we could have in mind are a dihedral domain with either a magnetic field parallel to the edge or a magnetic field orthogonal to the symmetry plane of the edge. This last situation can be used to investigate a lens with constant opening angle $\alpha \in(0, \pi)$ (see Figure 1). The following one term expansion is proved in [33, Theorem 8.12]:

$$
\lambda_{1}(h)=\nu(\alpha) h+O\left(h^{5 / 4}\right)
$$

where $\nu(\alpha)$ is the bottom of the spectrum of the magnetic Laplacian on an infinite wedge of opening $\alpha$ with a magnetic field orthogonal to the symmetry plane of the wedge.

- Problematics. In 3D and if $\Omega$ is smooth, a constant magnetic field is always tangent to the boundary at some points. When the boundary is not smooth, it may happen that the magnetic field is nowhere tangent to the boundary so that the main term in the spectral asymptotics is no more $\Theta_{0} h$ (see (1.4)). In our non regular case (as in the regular case), it is natural to understand how the geometry of the boundary combines with the magnetic field, especially since the magnetic field and the curvature are both 2 -forms. In the semiclassical framework, this leads to investigate how this combination of 2 -forms influences the localization (and microlocalization) properties of the eigenfunctions as much as their approximations by series in fractional powers of $h$. Even in the regular case considered in [25], the first term in the asymptotic expansion of the eigenfunctions is not obtained. In our paper we consider a case when the boundary is not smooth: the case when $\partial \Omega$ contains an edge. Our analysis will show that, even in the non smooth case, the repartition of the low lying eigenvalues is determined by an effective 1D harmonic oscillator with respect to the Fourier variable on the edge (see $[16,22,37,36,14])$.

- Structure of our result. Without going into the details, let us describe the structure of our main result. We prove in this paper that (see Theorem 1.16):

$$
\lambda_{n}(h) \underset{h \rightarrow 0}{\sim} \nu\left(\alpha_{0}\right) h+\left(\omega_{0}+(2 n-1) \omega_{1}\right) h^{3 / 2}+o\left(h^{3 / 2}\right),
$$

where:

- $\nu\left(\alpha_{0}\right)$ is the bottom of the spectrum of a model problem on a wedge of opening $\alpha_{0}$ (see Definition 1.3),

- $\alpha_{0}$ is the maximum opening angle of the edge (see Assumption 1.14),

- $\omega_{0} \in \mathbb{R}$ and $\omega_{1}>0$ are constants related to the geometry.

In contrast with the results from [7], we see that the splitting of the eigenvalues comes in the second term and not in the first term. Such a structure for the asymptotic expansion has already been observed in [22].

- Philosophy of the proofs. Let us now describe the philosophy of the proofs of asymptotic expansions for the magnetic Laplacian. Before explaining the general method that we implement in this paper, we distinguish between the different conceptual levels of our analysis. Our 
analysis uses the standard construction of quasimodes, localization techniques ("IMS" formula) and a priori estimates of Agmon type satisfied by the eigenfunctions. These "standard" tools, which are used in most of the papers dealing with $\lambda_{1}(h)$, are not enough to investigate $\lambda_{n}(h)$ due to the splitting arising at the second order. In fact such a fine behavior is the sign of a microlocal effect. In order to investigate this effect, we use a normal form procedure in the spirit of the Birkhoff normal form. It turns out that this normal form also strongly simplifies the construction of quasimodes. Once the behavior of the eigenfunctions in the phase space is established, we use the Feshbach-Grushin approach to reduce our operator to an electric Laplacian in the Born-Oppenheimer form (which itself can be analyzed through the FeshbachGrushin argument).

The first step to analyze such problems is to perform an accurate construction of quasimodes and to apply the spectral theorem. In other words we look for pairs $(\lambda, \psi)$ such that we have $\left\|\left(\mathcal{L}_{h}-\lambda\right) \psi\right\| \leq \varepsilon\|\psi\|$. Such pairs are constructed through an homogenization procedure involving different scalings with respect to the different variables. In particular the construction uses a formal power series expansion of the operator and an Ansatz in the same form for $(\lambda, \psi)$. The main difficulty in order to succeed is to choose the appropriate scalings. Another difficulty arising in this paper is due to the edge which obliges also to expand the Neumann boundary condition in power series.

The second step aims at giving a priori estimates satisfied by the eigenfunctions. These are localization estimates "à la Agmon" (see [1]). To prove them one generally needs to have a priori estimates for the eigenvalues which can be obtained with a partition of unity (see for instance [11]) and local comparisons with model operators. Then such a priori estimates involve an improvement in the asymptotic expansion of the eigenvalues. It turns out that, if we are just interested in the first terms of $\lambda_{1}(h)$, we do not need other tools to obtain what we are looking for (except maybe the introduction of functional calculus as in [25, Sections 11.2 and 13.2]).

In our paper we are interested in expansions at any order of $\lambda_{n}(h)$ so that we have to enlighten the underlying structure of the magnetic Laplacian which is comparatively deeper than the one of the electric Laplacian (where the classical harmonic approximation provides the asymptotics in generic cases, see [13]). To understand at which point the problem is different from the situation when we just want to know $\lambda_{1}(h)$, let us describe what is done for the 2D case in [16] (constant magnetic field) and in our recent work [36] (non constant magnetic field). In $[16,36]$ quasimodes are constructed and the usual localization estimates are proved. Then the behavior with respect to a phase variable needs to be determined to allow a reduction of dimension. Let us underline here that this phenomenon of phase localization is characteristic of the magnetic Laplacian and is intimately related to the structure of the low lying spectrum. In [16] Fournais and Helffer are led to use the pseudo-differential calculus and the Grushin formalism. In [36], the approach is structurally not the same (and this will be this approach that we will use in this paper, and which is used for instance in [14]). In [36], in the spirit of the Egorov theorem (see $[15,38,31]$ ), we use successive canonical transforms of the symbol of the operator corresponding to unitary transforms (change of gauge, change of variable, Fourier transform) and we reduce the operator, modulo remainders which are controlled thanks to the a priori estimates, to an electric Laplacian being in the Born-Oppenheimer form (see [10, 30] 
and more recently [8]). This reduction provides a rigorous explanation of the fact that, in the magnetic problems, the scalings corresponding to different variables are often different. In particular the present paper proves that, even in non regular cases and in $3 \mathrm{D}$, the reduction of the magnetic Laplacian to the electric Laplacian is possible.

Finally let us mention that the methods used in this paper are reminiscent of the semiclassical Birkhoff normal form (see for instance [39, 9, 40]).

1.2. Geometrical assumptions and local models. In this subsection we describe the geometry of the lens and the different models appearing in the analysis near the points of the boundary.

\subsubsection{Description of the lens. We first define the lens $\Omega$.}

Definition 1.2. Let $\Sigma$ be a smooth and connected surface in $\mathbb{R}^{3}$ and $\Pi$ be the plane $x_{3}=0$. We assume that the intersection $\Sigma \cap \Pi$ is a smooth and closed curve and that $\Sigma$ and $\Pi$ intersect neither normally nor tangentially. Denoting by $\Sigma^{+}$the set $\left\{\mathbf{x} \in \Sigma: x_{3}>0\right\}$ and by $\Sigma^{-}$its symmetric with respect to $x_{3}=0$, the lens $\Omega$ is the open set of the points lying between $\Sigma^{+}$ and $\Sigma^{-}$whereas the edge is

$$
E=\overline{\Sigma^{+}} \cap \overline{\Sigma^{-}} \text {. }
$$

We define $\alpha(\mathbf{x})$ as the opening angle between $\Sigma^{-}$and $\Sigma^{+}$at the point $\mathbf{x} \in E$. We assume that $\alpha(\mathbf{x}) \in(0, \pi)$ for all $\mathbf{x} \in E$.

In our situation the magnetic field $\boldsymbol{\beta}=(0,0,1)$ is normal to the plane where the edge lies. For $\mathbf{x} \in \partial \Omega \backslash E$ we introduce the angle $\theta(\mathbf{x})$ defined by:

$$
\boldsymbol{\beta} \cdot \mathbf{n}(\mathbf{x})=\sin \theta(\mathbf{x}) .
$$

A model lens with constant opening angle is given by two parts of a sphere glued together (see Figure 1). In this case we have

$$
\forall \mathbf{x} \in \partial \Omega \backslash E, \quad \frac{\pi-\alpha}{2}<\theta(\mathbf{x})
$$

where $\alpha \in(0, \pi)$ is the opening angle of the lens and we notice that the magnetic field is nowhere tangent to the boundary. In this paper we will assume that the opening angle of the lens is variable. For a given point $\mathrm{x}$ of the boundary, we analyze the localized (in a neighborhood of $\mathrm{x}$ ) magnetic Laplacian and we distinguish between $\mathrm{x}$ belonging to the edge and $\mathrm{x}$ belonging to the smooth part of the boundary.

1.2.2. Magnetic Laplacian in the half-space. Near the points of the regular boundary $\partial \Omega \backslash E$, we will be led to consider the magnetic Laplacian on the half-space with constant magnetic field, that is the Neumann realization on $\mathbb{R}_{+}^{3}=\left\{\left(y_{1}, y_{2}, y_{3}\right) \in \mathbb{R}^{3}: y_{3}>0\right\}$ of:

$$
H(\theta)=D_{y_{3}}^{2}+D_{y_{2}}^{2}+\left(D_{y_{1}}+y_{3} \cos \theta-y_{2} \sin \theta\right)^{2}
$$

the corresponding magnetic field given by $\boldsymbol{\beta}_{\theta}=(0, \cos \theta, \sin \theta)$ makes an angle $\theta \in\left[0, \frac{\pi}{2}\right]$ with the boundary. This operator has been widely studied (see for instance [29], [24] and more 

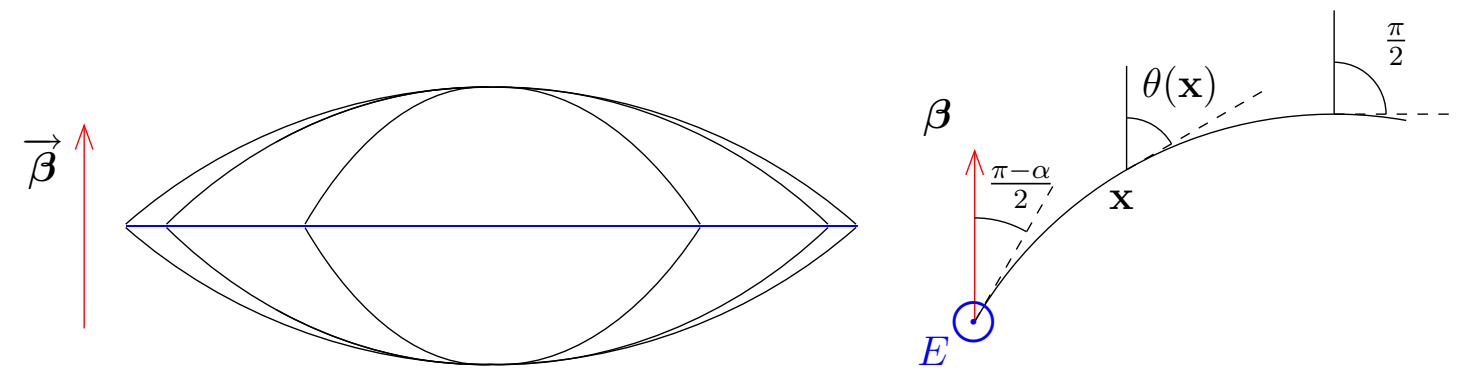

FIGURE 1. A lens $\Omega$ : the magnetic field is nowhere tangent to the boundary and it makes the angle $\theta(\mathbf{x})$ with the regular boundary.

recently [8]). The bottom of the spectrum of $H(\theta)$ is denoted by $\sigma(\theta)$. We recall (see [29]) that $\theta \mapsto \sigma(\theta)$ is an analytic function increasing from $\Theta_{0}=\sigma(0)$ to 1 .

1.2.3. Leading Operator. Let $\mathbf{x} \in E$ and $V$ a small neighborhood of $\mathbf{x}$ in $\Omega$. We suppose that the opening angle at $\mathrm{x}$ is $\alpha$. There is a diffeomorphism, denoted by the local coordinates $(\hat{s}, \hat{t}, \hat{z})$, from $V$ to an open subset of the infinite wedge of opening $\alpha$ :

$$
\mathcal{W}_{\alpha}=\mathbb{R} \times \mathcal{S}_{\alpha}
$$

where the 2D corner with fixed angle $\alpha \in(0, \pi)$ is defined by:

$$
\mathcal{S}_{\alpha}=\left\{(\hat{t}, \hat{z}) \in \mathbb{R}^{2}:|\hat{z}|<\hat{t} \tan \left(\frac{\alpha}{2}\right)\right\} .
$$

This diffeomorphism will be described in details in Section 2.
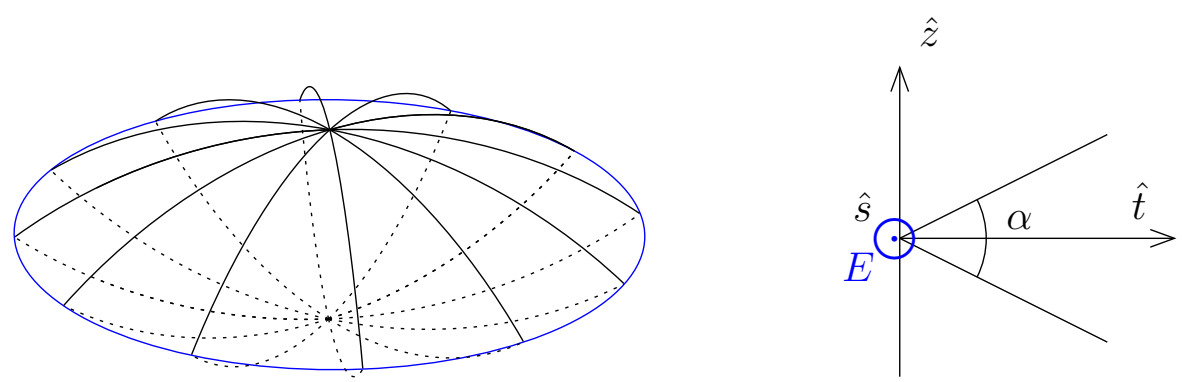

FIGURE 2. Using the local coordinates $(\hat{s}, \hat{t}, \hat{z})$, a neighborhood of a point of the edge can be described as a subset of the infinite wedge $\mathcal{W}_{\alpha}$.

Therefore we are led to study the operator $\mathfrak{L}_{\alpha}$ defined below.

Definition 1.3. Let $\mathfrak{L}_{\alpha}$ be the Neumann realization on $L^{2}\left(\mathcal{W}_{\alpha}, d \hat{s} d \hat{t} d \hat{z}\right)$ of

$$
D_{\hat{t}}^{2}+D_{\hat{z}}^{2}+\left(D_{\hat{s}}-\hat{t}\right)^{2} \text {. }
$$

We denote by $\nu(\alpha)$ the bottom of the spectrum of $\mathfrak{L}_{\alpha}$. 
Using the Fourier transform with respect to $\hat{s}$, we have the decomposition:

$$
\mathfrak{L}_{\alpha}=\int^{\oplus} L_{\alpha, \eta} d \eta,
$$

where $L_{\alpha, \eta}$ is the following Neumann realization on $L^{2}\left(\mathcal{S}_{\alpha}, d \hat{t} d \hat{z}\right)$ :

$$
L_{\alpha, \eta}=D_{\hat{t}}^{2}+D_{\hat{z}}^{2}+(\eta-\hat{t})^{2},
$$

where $\eta \in \mathbb{R}$ is the Fourier parameter. Let us remark that this operator admits the same form as the de Gennes operator (1.2). As

$$
\lim _{\substack{|(\hat{t}, \hat{z})| \rightarrow+\infty \\(\hat{t}, \hat{z}) \in \mathcal{S}_{\alpha}}}(\eta-\hat{t})^{2}=+\infty,
$$

the Schrödinger operator $L_{\alpha, \eta}$ has compact resolvent for all $(\alpha, \eta) \in(0, \pi) \times \mathbb{R}$.

Notation 1.4. For each $\alpha \in(0, \pi)$, we denote by $\nu(\alpha, \eta)$ the lowest eigenvalue of $L_{\alpha, \eta}$ and we denote by $u_{\alpha, \eta}$ a normalized corresponding eigenfunction.

Using (1.9) we have:

$$
\nu(\alpha)=\inf _{\eta \in \mathbb{R}} \nu(\alpha, \eta)
$$

- Properties related to $L_{\alpha, \eta}$ and $\mathfrak{L}_{\alpha}$. Let us gather a few elementary properties.

Lemma 1.5. We have:

(1) For all $(\alpha, \eta) \in(0, \pi) \times \mathbb{R}, \nu(\alpha, \eta)$ is a simple eigenvalue of $L_{\alpha, \eta}$.

(2) The function $(0, \pi) \times \mathbb{R} \ni(\alpha, \eta) \mapsto \nu(\alpha, \eta)$ is analytic.

(3) For all $\eta \in \mathbb{R}$, the function $(0, \pi) \ni \alpha \mapsto \nu(\alpha, \eta)$ is decreasing.

(4) The function $(0, \pi) \ni \alpha \mapsto \nu(\alpha)$ is non increasing.

(5) For all $\alpha \in(0, \pi)$, we have

$$
\lim _{\eta \rightarrow-\infty} \nu(\alpha, \eta)=+\infty \text { and } \lim _{\eta \rightarrow+\infty} \nu(\alpha, \eta)=\sigma\left(\frac{\pi-\alpha}{2}\right),
$$

where the function $\sigma$ is defined in Section 1.2.2.

Proof. We refer to [33, Section 3] for the two first statements. The monoticity comes from [33, Proposition 8.14] and the limits as $\eta$ goes to $\pm \infty$ are computed in [33, Theorem 5.2].

Remark 1.6. As $\nu(\pi)=\Theta_{0}$ (see Section 1.2.2), we have:

$$
\forall \alpha \in(0, \pi), \quad \nu(\alpha) \geq \Theta_{0} .
$$

Let us note that it is proved in [33, Proposition 8.13] that $\nu(\alpha)>\Theta_{0}$ for all $\alpha \in(0, \pi)$.

The following proposition is fundamental in order to compare the spectral quantities coming from the model operators:

Proposition 1.7. There exists $\tilde{\alpha} \in(0, \pi)$ such that for $\alpha \in(0, \tilde{\alpha})$, the function $\eta \mapsto \nu(\alpha, \eta)$ reaches its infimum and

$$
\nu(\alpha)<\sigma\left(\frac{\pi-\alpha}{2}\right)
$$


Proof. Using the normalized polar coordinates $(r, \phi) \in R_{0}:=\mathbb{R}_{+} \times\left(-\frac{1}{2}, \frac{1}{2}\right)$ and the gauge transform $\varphi(r, \phi)=\frac{r^{2}}{2} \phi$, we get (see [5]) that $L_{\alpha, \eta}$ is unitary equivalent to $L_{\alpha, \eta}^{\text {pol }}$ whose quadratic form is:

$$
Q_{\alpha, \eta}^{\mathrm{pol}}(u):=\int_{R_{0}}\left(\left|\partial_{r} u\right|^{2}+\frac{1}{\alpha^{2} r^{2}}\left|\partial_{\phi} u\right|^{2}+(r \cos (\alpha \phi)-\eta)^{2}|u|^{2}\right) r d r d \phi
$$

on the weighted space $L^{2}\left(R_{0}, r d r d \phi\right)$. We use a quasimode which does not depend on $\phi$ by taking

$$
u^{\mathrm{qm}}(r):=\frac{1}{4 \rho^{\mathrm{qm}}} e^{-\rho^{\mathrm{qm}} r^{2}}
$$

with $\rho^{\mathrm{qm}}:=\frac{1}{4} \sqrt{4-\pi}$. We take as Fourier parameter $\eta^{\mathrm{qm}}:=\frac{\pi^{2}}{4(4-\pi)}$. Computation yields

$$
\int_{R_{0}}\left(\left|\partial_{r} u^{\mathrm{qm}}(r)\right|^{2}+\left(r-\eta^{\mathrm{qm}}\right)^{2}\left|u^{\mathrm{qm}}(r)\right|^{2}\right) r d r d \phi=\sqrt{4-\pi} .
$$

Therefore $\left|Q_{\alpha, \eta^{\mathrm{qm}}}^{\mathrm{pol}}\left(u^{\mathrm{qm}}\right)-\sqrt{4-\pi}\right| \leq \alpha^{2} \int_{R_{0}}\left(r+\eta^{\mathrm{qm}}\right)\left|u^{\mathrm{qm}}(r)\right|^{2} r d r$ and by using the normalization $\int_{R_{0}}\left|u^{\mathrm{qm}}(r)\right|^{2} r d r d \phi=1$, the min-max principle provides

$$
\exists C^{\mathrm{qm}}>0, \quad \nu\left(\alpha, \eta^{\mathrm{qm}}\right) \leq \sqrt{4-\pi}+C^{\mathrm{qm}} \alpha^{2} .
$$

We get using (1.11):

$$
\forall \alpha \in(0, \pi), \quad \nu(\alpha) \leq \sqrt{4-\pi}+C^{\mathrm{qm}} \alpha^{2} .
$$

We also have the following inequality (see [24, Section 1.4]):

$$
\sqrt{\Theta_{0}^{2} \cos ^{2} \frac{\pi-\alpha}{2}+\sin ^{2} \frac{\pi-\alpha}{2}}<\sigma\left(\frac{\pi-\alpha}{2}\right)
$$

Since $\sqrt{4-\pi}+C^{\mathrm{qm}} \alpha^{2}<\sqrt{\Theta_{0}^{2} \cos ^{2} \frac{\pi-\alpha}{2}+\sin ^{2} \frac{\pi-\alpha}{2}}$ for $\alpha$ small enough, we get that there exists $\tilde{\alpha}$ such that (1.14) holds for $\alpha \in(0, \tilde{\alpha})$. Using (1.12), this upper bound shows that $\eta \mapsto \nu(\alpha, \eta)$ reaches its infimum for $\alpha \in(0, \tilde{\alpha})$. Remark that these computations can be found for more general magnetic fields in [33, Corollary 6.32]).

Remark 1.8. By computing $C^{\mathrm{qm}}$, we notice that (1.14) holds at least for $\alpha \in(0,1.2035)$. Numerical computations show that in fact (1.14) seems to hold for all $\alpha \in(0, \pi)$.

We will work under the following conjecture:

Conjecture 1.9. For all $\alpha \in(0, \pi), \eta \mapsto \nu(\alpha, \eta)$ has a unique critical point denoted by $\eta_{0}(\alpha)$ and it is a non degenerate minimum.

Remark 1.10. A numerical analysis seems to indicate that Conjecture 1.9 is true (see [33, Subsection 6.4.1]). Moreover standard spectral arguments ([33, Section 6.2]) make us think that this conjecture is true at least for small $\alpha$.

Under this conjecture and using the analytic implicit functions theorem, we deduce:

Lemma 1.11. Under Conjecture 1.9, the function $(0, \pi) \ni \alpha \mapsto \eta_{0}(\alpha)$ is analytic and so is $(0, \pi) \ni \alpha \mapsto \nu(\alpha)$. Moreover the function $(0, \pi) \ni \alpha \mapsto \nu(\alpha)$ is decreasing. 
1.2.4. Comparison between the models and choice of the lens $\Omega$. The previous subsections lead to compare the two quantities:

$$
\inf _{\mathbf{x} \in E} \nu(\alpha(\mathbf{x})), \quad \inf _{\mathbf{x} \in \partial \Omega \backslash E} \sigma(\theta(\mathbf{x})),
$$

where $\theta(\mathbf{x})$ is defined in (1.6), $\alpha(\mathbf{x})$ and $E$ are defined in Definition 1.2. Let us state the different assumptions under which we will work throughout this paper:

\section{Assumption 1.12.}

$$
\inf _{\mathbf{x} \in E} \nu(\alpha(\mathbf{x}))<\inf _{\mathbf{x} \in \partial \Omega \backslash E} \sigma(\theta(\mathbf{x})) .
$$

Remark 1.13. Using (1.7), the fact that $\sigma$ is increasing and Proposition 1.14, we check that, in the model case when $\Omega$ is made of two parts of a sphere glued together, Assumption 1.12 is satisfied for $\alpha$ small enough. By a continuity argument, Assumption 1.12 holds for not too large perturbations of this lens.

From the properties of the leading operator we see that we will be led to work near the point of the edge of maximal opening. Therefore we will assume the following generic assumption:

Assumption 1.14. We denote by $\alpha: E \mapsto(0, \pi)$ the opening angle of the lens. We assume that $\alpha$ admits a unique and non degenerate maximum at the point $\mathbf{x}_{0}$ and we let

$$
\alpha_{0}=\max _{E} \alpha .
$$

We denote $\tau=\tan \frac{\alpha}{2}$ and $\tau_{0}=\tan \frac{\alpha_{0}}{2}$.

1.3. Normal form. This is "classical" that Assumption 1.12 leads to localization properties of the eigenfunctions near the edge $E$ and more precisely near the points of the edge where $E \ni \mathbf{x} \mapsto \nu(\alpha(\mathbf{x}))$ is minimal. Therefore, since $\nu$ is decreasing and thanks to Assumption 1.14 , we expect that the first eigenfunctions concentrate near the point $\mathbf{x}_{0}$ where the opening is maximal. In Section 2, we explain how we can introduce, near each $\mathbf{x} \in E$, a local change of variables which transforms a neighborhood of $\mathbf{x}$ in $\Omega$ in a $\varepsilon_{0}$-neighborhood of $(0,0,0)$ of $\mathcal{W}_{\alpha(\mathbf{x})}$, denoted by $\mathcal{W}_{\alpha(\mathbf{x}), \varepsilon_{0}}$.

For the convenience of the reader, let us summarize the contents of Proposition 2.1: we write below the expression of the magnetic Laplacian in the new local coordinates $(\check{s}, \check{t}, \check{z})$ where $\check{s}$ is a curvilinear abscissa of the edge. The magnetic Laplacian $\mathcal{L}_{h}$ is given by the Laplace-Beltrami expression (on $L^{2}\left(|\check{G}|^{1 / 2} d \check{s} d \check{t} d \check{z}\right)$ ):

$$
\check{\mathcal{L}}_{h}:=|\check{G}|^{-1 / 2} \check{\nabla}_{h}|\check{G}|^{1 / 2} \check{G}^{-1} \check{\nabla}_{h}
$$

where:

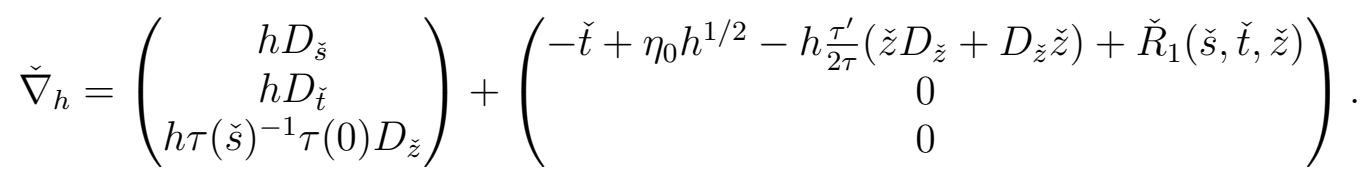

The forms of the Taylor expansions of the remainder $\check{R}_{1}$, the metric $\check{G}$ and the function $\check{s} \mapsto$ $\tau(\check{s})$ are analyzed in Proposition 2.1. 


\begin{tabular}{|l|l|l|}
\hline Variables & Domain & Operator \\
\hline \hline$\left(x_{1}, x_{2}, x_{3}\right)$ & Lens $\Omega$ & Magnetic laplacian $\mathcal{L}_{h}$ \\
\hline$(\check{s}, \check{t}, \check{z})$ & Wedge $\mathcal{W}_{\alpha}$ & Normal form $\check{\mathcal{L}}_{h}$ \\
\hline$(\hat{s}, \hat{t}, \hat{z})$ & Wedge $\mathcal{W}_{\alpha}$ & Rescaled operator $\widehat{\mathcal{L}}_{h}$ \\
\hline
\end{tabular}

TABLE 1. The magnetic Laplacian in the different coordinates

Remark 1.15. Such a normal form allows us to describe the leading structure of this magnetic Laplace-Beltrami operator. Indeed, if we just keep the main terms in (1.16) by neglecting formally the geometrical factors, our operator takes the simpler form:

$$
\left(h D_{\check{s}}-\check{t}+\eta_{0} h^{1 / 2}\right)^{2}+h^{2} D_{\check{t}}^{2}+h^{2} \tau(0)^{2} \tau(\check{s})^{-2} D_{\check{z}}^{2} .
$$

Performing another formal Taylor expansion near $\check{s}=0$, we are led to the following operator:

$$
\left(h D_{\check{s}}-\check{t}+\eta_{0} h^{1 / 2}\right)^{2}+h^{2} D_{\check{t}}^{2}+h^{2} D_{\check{z}}^{2}+c h^{2} \check{s}^{2} D_{\check{z}}^{2},
$$

where $c>0$. Using a scaling in Section 3, we get a rescaled operator $\widehat{\mathcal{L}}_{h}$ whose first term is the leading operator $\mathfrak{L}_{\alpha}$ and which allows to construct quasimodes. Moreover this form is suitable to establish microlocalization properties of the eigenfunctions with respect to $D_{\check{s}}$.

Table 1 summarizes the main expressions of the magnetic Laplacian in the different coordinates in which we are going to work throughout this paper.

1.4. Main result and contents. Our main result is a complete asymptotic expansion of all the first eigenvalues of $\mathcal{L}_{h}$ :

Theorem 1.16. We assume that Conjecture 1.9 is true. We also assume Assumptions 1.12 and 1.14. For all $n \geq 1$ there exists $\left(\mu_{j, n}\right)_{j \geq 0}$ such that we have:

$$
\lambda_{n}(h) \underset{h \rightarrow 0}{\sim} h \sum_{j \geq 0} \mu_{j, n} h^{j / 4} .
$$

Moreover, we have:

$$
\mu_{0, n}=\nu\left(\alpha_{0}\right), \quad \mu_{1, n}=0, \quad \mu_{2, n}=\omega_{0}+(2 n-1) \sqrt{\kappa \tau_{0}^{-1}\left\|D_{\hat{z}} u_{\eta_{0}}\right\|^{2} \partial_{\eta}^{2} \nu\left(\alpha_{0}, \eta_{0}\right)},
$$

where the geometrical constants $\omega_{0}$ and $\kappa$ are respectively given in (3.13) and (3.6).

Remark 1.17. Let us compare our result with the one of [25] recalled in (1.3). First we notice that due to Remark 1.6 the main term in our asymptotic expansion of $\lambda_{1}(h)$ is larger than $\Theta_{0} h$ and that the order of the second term $\left(h^{3 / 2}\right)$ is different. Secondly, as announced, we have the asymptotic expansions of all the $\lambda_{n}(h)$ and not only $\lambda_{1}(h)$. In particular, we see that the dependence of $n$ comes linearly in the second order term. Moreover, we observe that, for all $n \geq 1$, $\lambda_{n}(h)$ is simple for $h$ small enough. This simplicity, jointly with the quasimodes construction (see (3.21)), provides an approximation of the corresponding normalized eigenfunction. 
Remark 1.18. Let us underline that several non trivial insights (which combine part of the ideas from [16] and [36]) are used to obtain our main result. Starting from the rough techniques that have been used in the last fifteen years, we have proved, through an elementary Birkhoff normal form, basic microlocal arguments, the Feshbach-Grushin projection and the Born-Oppenheimer approximation, that we could approximately decouple the "phase" (controlled by explicit Fourier integral operators) and the "amplitude" (controlled by an effective electric Laplacian) of the first magnetic eigenfunctions.

- Organization of the proof. Theorem 1.16 is a direct consequence of Propositions 3.1 and 6.1. The paper is organized as follows. Section 2 is devoted to the definition of normal magnetic coordinates which give a normal form of $\mathcal{L}_{h}$. In Section 3, we prove Proposition 3.1 by using power series expansions of the normal form given in (1.16) and by constructing explicit quasimodes. In Section 4 we give a rough lower bound for $\lambda_{n}(h)$ and we prove that the associated eigenfunctions are localized in the sense of Agmon at the scale $h^{1 / 2}$ with respect to $(\check{t}, \check{z})$. In Section 5, we investigate the behavior of the eigenfunctions with respect to $D_{\check{s}}$ and we use a Grushin (cf. [20]) like approximation to describe the behavior of the eigenfunctions with respect to $\check{s}$. In Section 6 we combine the local and microlocal control of the eigenfunctions with the Grushin projection to reduce the study to the Born-Oppenheimer approximation and deduce Proposition 6.1.

\section{MAGNETIC NORMAL COORDINATES}

The aim of this section is to introduce normal coordinates near a point $\mathbf{x}_{1}$ of $E$. This normal form procedure is the key point in the spectral analysis of the magnetic Laplacian.

Proposition 2.1. For all $\mathrm{x}_{1} \in E$, there exist a neighborhood $\mathcal{V}$ of $\mathrm{x}_{1}$ in $\Omega, \varepsilon_{0}>0$ and local coordinates $(\check{s}, \check{t}, \check{z})$ such that $\mathcal{V}$ is given in the coordinates $(\check{s}, \check{t}, \check{z})=\Psi^{-1}(\mathbf{x})$ by:

$$
\mathcal{W}_{\alpha\left(\mathbf{x}_{1}\right), \varepsilon_{0}}:=\left\{(\check{s}, \check{t}, \check{z}) \in\left(-\varepsilon_{0}, \varepsilon_{0}\right) \times\left(0, \varepsilon_{0}\right) \times \mathbb{R}, \quad|\check{z}|<\tan \left(\frac{\alpha\left(\mathbf{x}_{1}\right)}{2}\right) \check{t}\right\} .
$$

and so that the edge $E \cap \mathcal{V}$ becomes $\left(-\varepsilon_{0}, \varepsilon_{0}\right) \times\{0\} \times\{0\}$ and is parametrized by $\check{s}$. Let us note:

$$
\tau(\check{s})=\tan \left(\frac{\alpha(\check{s})}{2}\right)
$$

where $\check{s} \mapsto \alpha(\check{s})$ is a parametrization of the opening angle $\alpha$ (see Definition 1.2).

There exists a metric $\breve{G}$ (written as a 3 by 3 matrix) such that the magnetic operator $\mathcal{L}_{h}$ (seen as acting on functions of its domain compactly supported in $\mathcal{V}$ ) becomes in these coordinates:

$$
\check{\mathcal{L}}_{h}=|\check{G}|^{-1 / 2} \check{\nabla}_{h} \mid \check{G}^{1 / 2} \check{G}^{-1} \check{\nabla}_{h}
$$


with boundary conditions:

$$
\begin{aligned}
& |\check{G}|^{1 / 2} \check{G}^{-1} \check{\nabla}_{h} \check{\psi} \cdot\left(\begin{array}{c}
-\tau^{\prime}(\check{s}) \check{t} \\
-\tau(\check{s}) \\
\pm 1
\end{array}\right)=0 \quad \text { on } \partial_{\mathrm{Neu}} \mathcal{W}_{\alpha\left(\mathbf{x}_{1}\right), \varepsilon_{0}} \\
& \check{\psi}=0 \quad \text { on } \partial_{\operatorname{Dir}} \mathcal{W}_{\alpha\left(\mathbf{x}_{1}\right), \varepsilon_{0}}
\end{aligned}
$$

where:

$$
\begin{aligned}
\partial_{\mathrm{Neu}} \mathcal{W}_{\alpha\left(\mathbf{x}_{1}\right), \varepsilon_{0}}= & \left\{(\check{s}, \check{t}, \check{z}) \in\left(-\varepsilon_{0}, \varepsilon_{0}\right) \times\left(0, \varepsilon_{0}\right) \times \mathbb{R}:|\check{z}|=\tau(\check{s}) \check{t}\right\}, \\
\partial_{\text {Dir }} \mathcal{W}_{\alpha\left(\mathbf{x}_{1}\right), \varepsilon_{0}}= & \left\{(\check{s}, \check{t}, \check{z}) \in\left\{ \pm \varepsilon_{0}\right\} \times\left[0, \varepsilon_{0}\right] \times \mathbb{R}:|\check{z}| \leq \tau\left( \pm \varepsilon_{0}\right) \check{t}\right\} \\
& \cup\left\{(\check{s}, \check{t}, \check{z}) \in\left[-\varepsilon_{0}, \varepsilon_{0}\right] \times\left\{\varepsilon_{0}\right\} \times \mathbb{R}:|\check{z}| \leq \tau(\check{s}) \varepsilon_{0}\right\},
\end{aligned}
$$

and:

$$
\check{\nabla}_{h}=\left(\begin{array}{c}
h D_{\check{s}} \\
h D_{\check{t}} \\
h \tau(\check{s})^{-1} \tau(0) D_{\check{z}}
\end{array}\right)+\left(\begin{array}{c}
-\check{t}+\eta_{0} h^{1 / 2}-h \frac{\tau^{\prime}}{2 \tau}\left(\check{z} D_{\check{z}}+D_{\check{z}} \check{z}\right)+\check{R}_{1}(\check{s}, \check{t}, \check{z}) \\
0 \\
0
\end{array}\right),
$$

where $\eta_{0}=\eta_{0}\left(\alpha_{0}\right)$ (see Conjecture 1.9). We will sometimes use the notation $\check{\mathcal{T}}_{h}:=|\check{G}|^{1 / 2} \check{G}^{-1} \check{\nabla}_{h}$.

Moreover the Taylor expansions of $\check{G}^{-1},|\check{G}|$ and $\check{R}_{1}$ can be written in the form:

$$
\begin{aligned}
& \check{G}^{-1}=\mathrm{Id}_{3}+\check{L}(\check{t}, \check{z})+(|\check{t}|+|\check{z}|) \mathcal{O}_{1}, \\
& |\check{G}|=1+\check{l}(\check{t}, \check{z})+(|\check{t}|+|\check{z}|) \mathcal{O}_{1}, \\
& \check{R}_{1}=\check{r}_{1}(\check{t}, \check{z})+\left(\check{t}^{2}+\check{z}^{2}\right) \mathcal{O}_{1},
\end{aligned}
$$

where $\mathrm{Id}_{3}$ is the identity matrix, $\check{r}_{1}$ is an homogeneous polynomial of degree 2 and where $\breve{L}$ and $\check{l}$ depend linearly on $(\check{t}, \check{z})$. We have used the notation $\mathcal{O}_{1}$ for a polynomial Taylor remainder in $(\check{s}, \check{t}, \check{z})$ whose terms are all of degree $\geq 1$.

Remark 2.2. If $\mathbf{x}_{1}$ is a point of maximal opening, we have $\tau^{\prime}(0)=0$. In particular for $\mathbf{x}_{1}=\mathbf{x}_{0}$, we have $\tau^{\prime}(0)=0$ and $\tau_{0}=\tau(0)$ (see Assumption 1.14).

To prove this proposition, we use successive change of variables (see Table 2).

\begin{tabular}{|l|l|l|}
\hline Variables & Domain & Section \\
\hline \hline$\left(x_{1}, x_{2}, x_{3}\right)$ & Lens $\Omega$ & \\
\hline$(s, t, z)$ & Gutter $\mathcal{G}_{\mathbf{x}_{1}}$ & Section 2.1 \\
\hline$(\breve{s}, \breve{t}, \breve{z})$ & Wedge with variable opening $G_{\mathbf{x}_{1}}$ & Section 2.2 \\
\hline$(\check{s}, \check{t}, \check{z})$ & Wedge with constant opening $\mathcal{W}_{\alpha}$ & Section 2.3 \\
\hline$(\hat{s}, \hat{t}, \hat{z})$ & Wedge with constant opening $\mathcal{W}_{\alpha}$ & Section 3.1 \\
\hline
\end{tabular}

TABLE 2. The changes of variables.

Let us notice that the last change of variables is just a rescaling. 
2.1. A first normalization: “the gutter". We consider the standard tubular coordinates (defined in a neighborhood of $(0,0,0))$ :

$$
\Phi(s, t, z)=(\gamma(s)+\operatorname{tn}(s), z)
$$

where $s \mapsto \gamma(s)$ is a normalized parametrization of the edge $E$ so that $(\gamma(0), 0)=\mathbf{x}_{1}$ and $n(s)$ is the inward pointing normal at the point $\gamma(s)$. We denote by $k(s)$ the algebraic curvature at the point $\gamma(s)$. The quadratic form associated to $\mathcal{L}_{h}$ (see (1.1)) writes:

$$
\mathcal{Q}_{h}(\psi)=\int_{\mathcal{G}_{\mathbf{x}_{1}, \varepsilon_{0}}}\left\{h^{2}\left|\partial_{t} \tilde{\psi}\right|^{2}+h^{2}\left|\partial_{z} \tilde{\psi}\right|^{2}+p^{-2}\left|\left(-i h \partial_{s}-t+\frac{k(s)}{2} t^{2}\right) \tilde{\psi}\right|^{2}\right\} p d s d t d z,
$$

where

$$
p(s, t)=1-t k(s),
$$

$\tilde{\psi}(s, t, z)=\psi(\Phi(s, t, z))$ is supported near $(0,0,0)$ and where the local "gutter" $\mathcal{G}_{\mathbf{x}_{1}, \varepsilon_{0}}$ is defined as:

$$
\mathcal{G}_{\mathbf{x}_{1}, \varepsilon_{0}}=\left\{(s, t, z) \in\left(-\varepsilon_{0}, \varepsilon_{0}\right) \times\left(0, \varepsilon_{0}\right) \times \mathbb{R}:-f_{\mathbf{x}_{1}}(s, t)<z<g_{\mathbf{x}_{1}}(s, t)\right\},
$$

where $f_{\mathbf{x}_{1}}$ and $g_{\mathbf{x}_{1}}$ are smooth functions. In particular, this is clear by the local inversion theorem that, if $\varepsilon_{0}$ is chosen small enough, there exists a neighborhood $\mathcal{V}$ of $\mathrm{x}_{1}$ such that $\Phi^{-1}(\mathcal{V})=\mathcal{G}_{\mathbf{x}_{1}, \varepsilon_{0}}$.

\subsection{From the gutter to the edge.}

2.2.1. The change of variables $J$. We now want to transform the integration domain in an edge with variable angle. In Definition 1.2, we have assumed that the lens is symmetric. However, we provide below a general change of variables which can also work for a non symmetric lens.

We introduce the rotation $R_{\frac{\alpha(s)}{2}}$ with angle $\frac{\alpha(s)}{2}$ and we let:

$$
(u, v)=R_{\frac{\alpha(s)}{2}}(t, z),
$$

that is:

$$
\left\{\begin{array}{l}
u=\cos \left(\frac{\alpha(s)}{2}\right) t-\sin \left(\frac{\alpha(s)}{2}\right) z \\
v=\sin \left(\frac{\alpha(s)}{2}\right) t+\cos \left(\frac{\alpha(s)}{2}\right) z
\end{array}\right.
$$

Therefore, near $(0,0,0)$, the Neumann boundary is:

$$
\left\{(s, u, v): \phi_{s}(u)=v \text { or } u=\psi_{s}(v)\right\}
$$

where $\phi_{s}$ et $\psi_{s}$ are smooth functions (also smoothly depending on the parameter $s$ ) satisfying:

$$
\phi_{s}(0)=\psi_{s}(0)=0, \quad \phi_{s}^{\prime}(0)=0, \quad \psi_{s}^{\prime}(0)=\cot \alpha(s) .
$$

We now introduce the change of variables

$$
(\breve{u}, \breve{v})=C_{s}(u, v)
$$



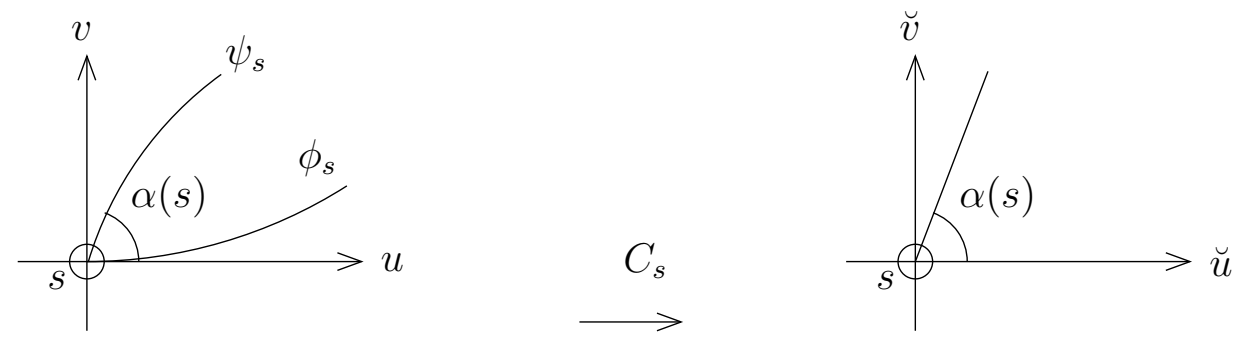

FIgURE 3. Change of variables $C_{s}$

defined by:

$$
\left\{\begin{array}{l}
\breve{u}=u-\psi_{s}(v)+\cot \alpha(s)\left(v-\phi_{s}(u)\right) \\
\breve{v}=v-\phi_{s}(u) .
\end{array}\right.
$$

In particular, the Neumann boundary becomes the union of $\breve{v}=0$ and $\breve{v}=\tan \alpha(s) \breve{u}$. We have:

$$
d_{u, v} C_{s}=\left(\begin{array}{cc}
1-\cot \alpha(s) \phi_{s}^{\prime}(u) & \cot \alpha(s)-\psi_{s}^{\prime}(v) \\
-\phi_{s}^{\prime}(u) & 1
\end{array}\right)=I_{2}+R_{s}(u, v),
$$

where

$$
R_{s}(u, v)=\left(\begin{array}{cc}
-\cot \alpha(s) \phi_{s}^{\prime}(u) & \cot \alpha(s)-\psi_{s}^{\prime}(v) \\
-\phi_{s}^{\prime}(u) & 0
\end{array}\right) .
$$

We have $R_{s}(0,0)=0$ so that $C_{s}$ defines a local diffeomorphism. We use now the inverse rotation and we consider $(\breve{t}, \breve{z})=R_{-\frac{\alpha(s)}{2}}(\breve{u}, \breve{v})$ :

$$
\left\{\begin{array}{l}
\breve{t}=\cos \left(\frac{\alpha(s)}{2}\right) \breve{u}+\sin \left(\frac{\alpha(s)}{2}\right) \breve{v}, \\
\breve{z}=-\sin \left(\frac{\alpha(s)}{2}\right) \breve{u}+\cos \left(\frac{\alpha(s)}{2}\right) \breve{v} .
\end{array}\right.
$$

We define:

$$
J(s, t, z)=\left(s, R_{-\frac{\alpha(s)}{2}} C_{s} R_{\frac{\alpha(s)}{2}}(t, z)\right)=(\breve{s}, \breve{t}, \breve{z}) .
$$

There exists a neighborhood $W$ of $(0,0,0)$ which is sent by $J$ on the straight gutter with variable opening $G_{\mathbf{x}_{1}, \varepsilon_{0}}$ defined by:

$$
G_{\mathbf{x}_{1}, \varepsilon_{0}}:=\left\{(\breve{s}, \breve{t}, \breve{z}) \in\left(-\varepsilon_{0}, \varepsilon_{0}\right) \times\left(0, \varepsilon_{0}\right) \times \mathbb{R}, \quad|\breve{z}|<\tan \left(\frac{\alpha(\breve{s})}{2}\right) \breve{t}\right\} .
$$

2.2.2. Jacobian of $J$. In this subsection we describe the Taylor expansion of the local diffeomorphism $J$. A Taylor expansion of $C_{s}(u, v)$ near $(u, v)=(0,0)$ provides:

$$
\left\{\begin{array}{l}
\breve{u}=u-\frac{\psi_{s}^{\prime \prime}(0)}{\phi^{\prime 2}} v^{2}-\cot \alpha(s) \frac{\phi_{s}^{\prime \prime}(0)}{2} u^{2}+O\left(|u|^{3}+|v|^{3}\right) \\
\breve{v}=v-\frac{\frac{\phi_{s}^{\prime}(0)}{2}}{2} u^{2}+O\left(|u|^{3}+|v|^{3}\right),
\end{array}\right.
$$

where the $O$ smoothly depends on $s$. We deduce:

$$
d_{s, t, z} J=\left(\begin{array}{cc}
1 & 0 \\
W_{2}(s, t, z) & \operatorname{ld}_{2}+W_{1}(s, t, z)
\end{array}\right),
$$


where $W_{1}(s, t, z)=P_{1}(t, z)+(|\check{t}|+|\check{z}|) \mathcal{O}_{1}, W_{2}(s, t, z)=P_{2}(t, z)+\left(\check{t}^{2}+\check{z}^{2}\right) \mathcal{O}_{1}$ and where the coefficients of the matrices $P_{j}$ are homogeneous polynomials of order $j$. We can also write the Taylor expansions:

$$
\left\{\begin{array}{l}
t=\breve{t}+U(\breve{t}, \breve{z})+\left(\check{t}^{2}+\check{z}^{2}\right) \mathcal{O}_{1} \\
z=\breve{z}+V(\breve{t}, \breve{z})+\left(\check{t}^{2}+\breve{z}^{2}\right) \mathcal{O}_{1}
\end{array},\right.
$$

where $U, V$ are homogeneous of order 2 .

2.2.3. Expression of the quadratic form in the coordinates $(\breve{s}, \breve{t}, \breve{z})$. In the coordinates $(\breve{s}, \breve{t}, \breve{z})$ the quadratic form becomes:

$$
\mathcal{Q}_{h}(\psi)=\breve{\mathcal{Q}}_{h}(\breve{\psi})=\left\langle\breve{G}^{-1} \breve{\nabla}_{h} \breve{\psi}, \breve{\nabla}_{h} \breve{\psi}\right\rangle_{L^{2}\left(|\breve{G}|^{1 / 2} d \breve{s} d \breve{t} d \breve{z}\right)}
$$

with:

$$
\breve{G}^{-1}=(d J)\left(\begin{array}{ccc}
p^{-2} & 0 & 0 \\
0 & 1 & 0 \\
0 & 0 & 1
\end{array}\right){ }^{t}(d J)
$$

and:

$$
\breve{\nabla}_{h}=\left(\begin{array}{c}
h \partial_{\breve{s}} \\
h \partial_{\breve{t}} \\
h \partial_{\breve{z}}
\end{array}\right)+{ }^{t}(d J)^{-1}\left(\begin{array}{c}
-\breve{t}+\breve{R}(\breve{s}, \breve{t}, \breve{z}) \\
0 \\
0
\end{array}\right)
$$

where $\breve{R}$ satisfies $\breve{R}=\breve{S}+\left(\breve{t}^{2}+\breve{z}^{2}\right) \mathcal{O}_{1}$ where $\breve{S}$ is an homogeneous polynomial of degree 2 depending on $(\breve{t}, \breve{z})$. This becomes:

$$
\breve{\nabla}_{h}=\left(\begin{array}{c}
h \partial_{\breve{s}} \\
h \partial_{\breve{t}} \\
h \partial_{\breve{z}}
\end{array}\right)+\left(\begin{array}{c}
-\breve{t}+\breve{R}_{1}(\breve{s}, \breve{t}, \breve{z}) \\
0 \\
0
\end{array}\right)
$$

where $\breve{R}_{1}$ satisfies:

$$
\breve{R}_{1}=\breve{S}_{1}(\breve{t}, \breve{z})+\left(\breve{t}^{2}+\check{z}^{2}\right) \mathcal{O}_{1}
$$

where $\breve{S}_{1}$ is homogeneous of order 2 . We have (see (2.6)):

$$
p^{-2}(\breve{s}, \breve{t})=1+2 k(0) \breve{t}+(|\breve{s}|+|\breve{t}|) \mathcal{O}_{1} .
$$

By using (2.12), (2.15) and (2.16) we get the approximation:

$$
\breve{G}^{-1}=\mathrm{Id}_{3}+\left(\begin{array}{cc}
2 k(0) \breve{t} & 0 \\
0 & W_{1}+{ }^{t} W_{1}
\end{array}\right)+(|\check{t}|+|\check{z}|) \mathcal{O}_{1} .
$$

Therefore the metrics $\breve{G}^{-1}$ takes the form:

$$
\breve{G}^{-1}=I_{3}+\breve{L}+(|\check{t}|+|\check{z}|) \mathcal{O}_{1}
$$

and:

$$
|\breve{G}|=1+\breve{l}+(|\check{t}|+|\check{z}|) \mathcal{O}_{1}
$$


where $\breve{l}$ and $\breve{L}$ are linear expressions in $(\breve{t}, \breve{z})$. The normal to the Neumann boundary is given by the vector: $\left(\begin{array}{c}-\tau^{\prime}(\breve{s}) \breve{t} \\ -\tau(\breve{s}) \\ \pm 1\end{array}\right)$ where $\tau$ is defined in (2.2) so that the boundary conditions take the form:

$$
\begin{aligned}
& |\breve{G}|^{1 / 2} \breve{G}^{-1} \breve{\nabla}_{h} \breve{\psi} \cdot\left(\begin{array}{c}
-\tau^{\prime}(\breve{s}) \breve{t} \\
-\tau(\breve{s}) \\
\pm 1
\end{array}\right)=0 \quad \text { on } \partial_{\mathrm{Neu}} G_{\mathbf{x}_{1}, \varepsilon_{0}} \\
& \check{\psi}=0 \quad \text { on } \partial_{\mathrm{Dir}} G_{\mathbf{x}_{1}, \varepsilon_{0}} \text {. }
\end{aligned}
$$

2.3. From $G_{\mathbf{x}_{1}, \varepsilon_{0}}$ to the edge with constant opening $\mathcal{W}_{\alpha\left(\mathbf{x}_{1}\right), \varepsilon_{0}}$. Finally we use the scaling:

$$
\check{s}=\breve{s}, \quad \check{t}=\breve{t}, \quad \check{z}=\tau(\breve{s})^{-1} \tau(0) \breve{z} .
$$

In particular, we have:

$$
\partial_{\breve{s}}=\partial_{\check{s}}-\frac{\tau^{\prime}(\check{s})}{\tau(\check{s})} \check{z} \partial_{\check{z}} \text {. }
$$

We also perform the canonical change of function (see for instance [14, Section 2.1] and also [26, Theorem 18.5.9]):

$$
\check{\psi}(\check{s}, \check{t}, \check{z})=\tau(\check{s})^{1 / 2} \tau(0)^{-1 / 2} \breve{\psi}\left(\check{s}, \check{t}, \tau(\check{s}) \tau(0)^{-1} \check{z}\right)
$$

to make the weight related to the Jacobian to disappear. A computation provides:

$$
\tau(\check{s})^{1 / 2} \tau(0)^{-1 / 2}\left(\partial_{\check{s}}-\frac{\tau^{\prime}(\check{s})}{\tau(\check{s})} \check{z} \partial_{\check{z}}\right) \tau(\check{s})^{-1 / 2} \tau(0)^{1 / 2}=\partial_{\check{s}}-\frac{\tau^{\prime}}{2 \tau}\left(\check{z} \partial_{\check{z}}+\partial_{\check{z}} \check{z}\right) .
$$

Making a change of gauge by conjugating the operator by $e^{-i h^{-1 / 2} \eta_{0} \check{s}}$ (see Conjecture 1.9), the quadratic form takes the form:

$$
\mathcal{Q}_{h}(\psi)=\check{\mathcal{Q}}_{h}(\check{\psi})=\left\langle\check{G}^{-1} \check{\nabla}_{h} \check{\psi}, \check{\nabla}_{h} \check{\psi}\right\rangle_{L^{2}\left(|\check{G}|^{1 / 2} d \check{s} d \check{d} d \check{z}\right)},
$$

where:

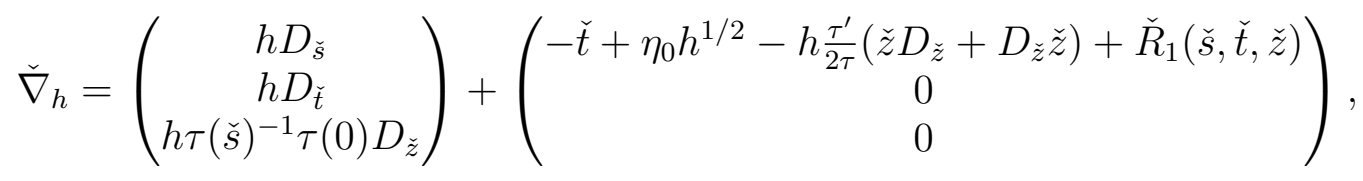

where $\check{R}_{1}$ satisfies:

$$
\check{R}_{1}=r_{1}(\check{t}, \check{z})+\left(\check{t}^{2}+\check{z}^{2}\right) \mathcal{O}_{1}
$$

where $r_{1}$ is homogeneous of order 2 . The associated operator on $L^{2}\left(\mathcal{W}_{\alpha\left(\mathbf{x}_{1}\right), \varepsilon_{0}},|\check{G}|^{1 / 2} d \check{s} d \check{t} d \check{z}\right)$ is given by the Laplace-Beltrami expression:

$$
|\check{G}|^{-1 / 2} \check{\nabla}_{h}|\check{G}|^{1 / 2} \check{G}^{-1} \check{\nabla}_{h}
$$

with boundary conditions:

$$
\begin{aligned}
& |\check{G}|^{1 / 2} \check{G}^{-1} \check{\nabla}_{h} \check{\psi} \cdot \check{\mathbf{n}}=0 \quad \text { on } \partial_{\mathrm{Neu}} \mathcal{W}_{\alpha\left(\mathbf{x}_{1}\right), \varepsilon_{0}} \\
& \check{\psi}=0 \quad \text { on } \partial_{\mathrm{Dir}} \mathcal{W}_{\alpha\left(\mathbf{x}_{1}\right), \varepsilon_{0}}
\end{aligned}
$$


where

$$
\check{\mathbf{n}}=\left(\begin{array}{c}
-\tau^{\prime}(\check{s}) \check{t} \\
-\tau(\check{s}) \\
\pm 1
\end{array}\right)
$$

\section{QUASIMODES}

This section is devoted to the proof of the following proposition.

Proposition 3.1. We assume that Conjecture 1.9 is true. We also assume Assumptions 1.12 and 1.14. For all $n \geq 1$ and $J \geq 1$, there exist $h_{0}>0$ and $C>0$ such that, for $h \in\left(0, h_{0}\right)$ :

$$
d\left(h \sum_{j=0}^{J} \mu_{j, n} h^{j / 4}, \mathfrak{S}\left(\mathcal{L}_{h}\right)\right) \leq C h h^{\frac{J+1}{4}},
$$

where the $\mu_{j, n}$ are defined in Theorem 1.16 and $d$ is the usual distance.

The main idea in this section is to implement an homogenization procedure and a formal power series expansion of the operator $\check{\mathcal{L}}_{h}$ (acting on $L^{2}\left(\mathcal{W}_{\alpha},|G|^{1 / 2} d \check{s} d \check{t} d \check{z}\right)$ ) and of the boundary operator $\check{\mathcal{T}}_{h}$ described in Proposition 2.1. We use the normal form given in Proposition 2.1 at the point $\mathbf{x}_{1}=\mathbf{x}_{0}$ (see Assumption 1.14 and Remark 2.2). First we give relations between spectral quantities associated to the model operator $L_{\alpha, \eta}$ defined in (1.10):

- Feynman-Hellmann Theorems. Thanks to the analytic dependence with respect to $\alpha$, we get the two following propositions (the proof is standard, see for instance [27] and more recently [14]).

Notation 3.2. In order to shorten the notation we will denote by $\eta_{0}$ the number $\eta_{0}\left(\alpha_{0}\right)$ (see Conjecture 1.9) and by $u_{\eta_{0}}:=u_{\alpha_{0}, \eta_{0}}$ the associated eigenfunction for the operator $L_{\alpha_{0}, \eta_{0}}$ defined in (1.10). We also introduce the functions

$$
v_{\eta_{0}}:=\left(\partial_{\eta} u_{\alpha_{0}, \eta}\right)_{\eta=\eta_{0}} \quad \text { and } \quad w_{\eta_{0}}:=\left(\partial_{\eta}^{2} u_{\alpha_{0}, \eta}\right)_{\eta=\eta_{0}} .
$$

Thanks to the analytic dependence with respect to $\alpha$, we get the two following propositions (the proof is standard, see for instance [27] and more recently [14]).

Proposition 3.3. We have:

$$
\left(L_{\alpha_{0}, \eta_{0}}-\nu\left(\alpha_{0}, \eta_{0}\right)\right) v_{\eta_{0}}=-2\left(\eta_{0}-\hat{t}\right) u_{\eta_{0}} .
$$

Proposition 3.4. We have:

$$
\left.\left(L_{\alpha_{0}, \eta_{0}}-\nu\left(\alpha_{0}, \eta_{0}\right)\right) w_{\eta_{0}}=\left(\partial_{\eta}^{2} \nu\left(\alpha_{0}, \eta_{0}\right)-2\right) u_{\eta_{0}}-4\left(\eta_{0}-\hat{t}\right) v_{\eta_{0}}\right)
$$

3.1. Quasimodes for the normal form. Before starting the analysis, we use the following scaling which keeps $\mathcal{W}_{\alpha}$ invariant:

$$
\check{s}=h^{1 / 4} \hat{s}, \quad \check{t}=h^{1 / 2} \hat{t}, \quad \check{z}=h^{1 / 2} \hat{z}
$$

so that we denote by $\widehat{\mathcal{L}}_{h}$ and $\widehat{\mathcal{T}}_{h}$ the operators $h^{-1} \check{\mathcal{L}}_{h}$ and $h^{-1 / 2} \check{\mathcal{T}}_{h}$ in the coordinates $(\hat{s}, \hat{t}, \hat{z})$. 
Using the Taylor expansions written in Proposition 2.1, we can write in the sense of formal power series the magnetic Laplacian near the edge and the associated magnetic Neumann boundary condition:

$$
\widehat{\mathcal{L}}_{h} \underset{h \rightarrow 0}{\sim} \sum_{j \geq 0} \mathcal{L}_{j} h^{j / 4}
$$

and

$$
\widehat{\mathcal{T}}_{h} \underset{h \rightarrow 0}{\sim} \sum_{j \geq 0} \mathcal{T}_{j} h^{j / 4}
$$

where the first $\mathcal{L}_{j}$ and $\mathcal{T}_{j}$ are given by:

$$
\begin{aligned}
& \mathcal{L}_{0}=D_{\hat{t}}^{2}+D_{\hat{z}}^{2}+\left(\hat{t}-\eta_{0}\right)^{2}, \\
& \mathcal{L}_{1}=-2\left(\hat{t}-\eta_{0}\right) D_{\hat{s}} \\
& \mathcal{L}_{2}=D_{\hat{s}}^{2}+2 \kappa \tau_{0}^{-1} \hat{s}^{2} D_{\hat{z}}^{2}+L_{2},
\end{aligned}
$$

where

$$
L_{2}=2\left(\eta_{0}-\hat{t}\right) \hat{r}_{1}-\frac{\hat{l}}{2} \hat{P} \hat{P}+\hat{P} \frac{\hat{l}}{2} \hat{P}+\hat{P} \hat{L} \hat{P}, \quad \hat{P}=\left(\begin{array}{c}
\eta_{0}-\hat{t} \\
D_{\hat{t}} \\
D_{\hat{z}}
\end{array}\right)
$$

and:

$$
\begin{aligned}
& \mathcal{T}_{0}=\left(-\hat{t}+\eta_{0}, D_{\hat{t}}, D_{\hat{z}}\right), \\
& \mathcal{T}_{1}=\left(D_{\hat{s}}, 0,0\right), \\
& \mathcal{T}_{2}=\left(0,0, \kappa \tau_{0}^{-1} \hat{s}^{2} D_{\hat{z}}\right)+\frac{\hat{l}}{2} \hat{P}+\hat{L} \hat{P},
\end{aligned}
$$

with

$$
\kappa=-\frac{\tau^{\prime \prime}(0)}{2}>0
$$

where $\tau$ is defined in (2.2). We recall that $\tau_{0}=\tau(0)$. We have used the notation

$$
\begin{aligned}
& \hat{r}_{1}(\hat{t}, \hat{z})=h^{-1} \check{r}_{1}\left(h^{1 / 2} \hat{t}, h^{1 / 2} \hat{z}\right), \\
& \hat{l}(\hat{t}, \hat{z})=h^{-1 / 2} \check{l}\left(h^{-1 / 2} \hat{t}, h^{-1 / 2} \hat{z}\right), \\
& \hat{L}(\hat{t}, \hat{z})=h^{-1 / 2} \check{L}\left(h^{-1 / 2} \hat{t}, h^{-1 / 2} \hat{z}\right) .
\end{aligned}
$$

We will also use an asymptotic expansion of the normal $\hat{\mathbf{n}}(h)$. We recall (see (2.19)) that we have $\check{\mathbf{n}}=\left(-\tau^{\prime}(\check{s}) \check{t},-\tau(\check{s}), \pm 1\right)$ so that we get:

$$
\hat{\mathbf{n}}(h) \underset{h \rightarrow 0}{\sim} \sum_{j \geq 0} \mathbf{n}_{j} h^{j / 4},
$$

with:

$$
\mathbf{n}_{0}=\left(0,-\tau_{0}, \pm 1\right), \quad \mathbf{n}_{1}=(0,0,0), \quad \mathbf{n}_{2}=\left(0, \kappa \hat{s}^{2}, 0\right)
$$


We look for $(\hat{\lambda}(h), \hat{\psi}(h))$ in the form:

$$
\begin{aligned}
& \hat{\lambda}(h) \underset{h \rightarrow 0}{\sim} \sum_{j \geq 0} \mu_{j} h^{j / 4}, \\
& \hat{\psi}(h) \underset{h \rightarrow 0}{\sim} \sum_{j \geq 0} \psi_{j} h^{j / 4},
\end{aligned}
$$

which satisfies, in the sense of formal series, the following boundary value problem:

$$
\left\{\begin{aligned}
\widehat{\mathcal{L}}(h) \hat{\psi}(h) \underset{h \rightarrow 0}{\sim} \hat{\lambda}(h) \hat{\psi}(h), \\
\hat{\mathbf{n}}(h) \cdot \widehat{\mathcal{T}}_{h} \hat{\psi}(h) \underset{h \rightarrow 0}{\sim} 0 \quad \text { on } \quad \partial_{\text {Neu }} \mathcal{W}_{\alpha_{0}} .
\end{aligned}\right.
$$

This provides an infinite system of PDE's.

- Terms in $h^{0}$. We solve the equation:

$$
\mathcal{L}_{0} \psi_{0}=\mu_{0} \psi_{0}, \text { in } \mathcal{W}_{\alpha_{0}}, \quad \mathbf{n}_{0} \cdot \mathcal{T}_{0} \psi_{0}=0 \text {, on } \partial_{\text {Neu }} \mathcal{W}_{\alpha_{0}} .
$$

We notice that the boundary condition is exactly the Neumann condition. We are led to choose $\mu_{0}=\nu\left(\alpha_{0}, \eta_{0}\right)$ and $\psi_{0}(\hat{s}, \hat{t}, \hat{z})=u_{\eta_{0}}(\hat{t}, \hat{z}) f_{0}(\hat{s})$ (see Notation 3.2) where $f_{0}$ will be chosen (in the Schwartz class) in a next step.

- Terms in $h^{1 / 4}$. Collecting the terms of size $h^{1 / 4}$, we find the equation:

$$
\left(\mathcal{L}_{0}-\mu_{0}\right) \psi_{1}=\left(\mu_{1}-\mathcal{L}_{1}\right) \psi_{0}, \quad \mathbf{n}_{0} \cdot \mathcal{T}_{0} \psi_{1}=0, \text { on } \partial_{\text {Neu }} \mathcal{W}_{\alpha_{0}} .
$$

As in the previous step, the boundary condition is just the Neumann condition. We use Proposition 3.3 and we deduce:

$$
\left(\mathcal{L}_{0}-\mu_{0}\right)\left(\psi_{1}+v_{\eta_{0}}(\hat{t}, \hat{z}) D_{\hat{s}} f_{0}(\hat{s})\right)=\mu_{1} \psi_{0}, \quad \mathbf{n}_{0} \cdot \mathcal{T}_{0} \psi_{1}=0, \text { on } \partial_{\mathrm{Neu}} \mathcal{W}_{\alpha_{0}}
$$

Taking the scalar product of the r.h.s. of the first equation with $u_{\eta_{0}}$ with respect to $(\hat{t}, \hat{z})$ and using the Neumann boundary condition for $v_{\eta_{0}}$ and $\psi_{1}$ when integrating by parts, we find $\mu_{1}=0$. This leads to choose:

$$
\psi_{1}(\hat{s}, \hat{t}, \hat{z})=v_{\eta_{0}}(\hat{t}, \hat{z}) D_{\hat{s}} f_{0}(\hat{s})+f_{1}(\hat{s}) u_{\eta_{0}}(\hat{t}, \hat{z}),
$$

where $f_{1}$ will be determined in a next step.

- Terms in $h^{1 / 2}$. Let us now deal with the terms of order $h^{1 / 2}$ :

$$
\left(\mathcal{L}_{0}-\mu_{0}\right) \psi_{2}=\left(\mu_{2}-\mathcal{L}_{2}\right) \psi_{0}-\mathcal{L}_{1} \psi_{1}, \quad \mathbf{n}_{0} \cdot \mathcal{T}_{0} \psi_{2}=-\mathbf{n}_{0} \cdot \mathcal{T}_{2} \psi_{0}-\mathbf{n}_{2} \cdot \mathcal{T}_{0} \psi_{0}, \text { on } \partial_{\text {Neu }} \mathcal{W}_{\alpha_{0}}
$$

We analyze the boundary condition:

$$
\begin{aligned}
\mathbf{n}_{0} \cdot \mathcal{T}_{2} \psi_{0}+\mathbf{n}_{2} \cdot \mathcal{T}_{0} \psi_{0} & = \pm \kappa \tau_{0}^{-1} \hat{s}^{2} D_{\hat{z}} \psi_{0}+\kappa \hat{s}^{2} D_{\hat{t}} \psi_{0}+\mathbf{n}_{0} \cdot \frac{\hat{l}}{2} \hat{P} \psi_{0}+\mathbf{n}_{0} \cdot \hat{L} \hat{P} \psi_{0} \\
& =\kappa \tau_{0}^{-1} \hat{s}^{2}\left( \pm D_{\hat{z}}+\tau_{0} D_{\hat{t}}\right) \psi_{0}+\mathbf{n}_{0} \cdot \frac{\hat{l}}{2} \hat{P} \psi_{0}+\mathbf{n}_{0} \cdot \hat{L} \hat{P} \psi_{0} \\
& = \pm 2 \kappa \tau_{0}^{-1} \hat{s}^{2} D_{\hat{z}} \psi_{0}+\mathbf{n}_{0} \cdot \frac{\hat{l}}{2} \hat{P} \psi_{0}+\mathbf{n}_{0} \cdot \hat{L} \hat{P} \psi_{0} .
\end{aligned}
$$


where we have used the Neumann boundary condition of $\psi_{0}$. Then, we use Proposition 3.4 together with (3.3) and (3.4) to get:

$$
\left(\mathcal{L}_{0}-\mu_{0}\right)\left(\psi_{2}-v_{\eta_{0}} D_{\hat{s}} f_{1}-\frac{w_{\eta_{0}}}{2} D_{\hat{s}}^{2} f_{0}\right)=\mu_{2} \psi_{0}-\frac{\partial_{\eta}^{2} \nu\left(\alpha_{0}, \eta_{0}\right)}{2} D_{\hat{s}}^{2} \psi_{0}-2 \kappa \tau_{0}^{-1} \hat{s}^{2} D_{\hat{z}}^{2} \psi_{0}-L_{2} \psi_{0},
$$

with boundary condition:

$$
\mathbf{n}_{0} \cdot \mathcal{T}_{0} \psi_{2}=\mp 2 \kappa \hat{s}^{2} \tau_{0}^{-1} D_{\hat{z}} \psi_{0}-\mathbf{n}_{0} \cdot \frac{\hat{l}}{2} \hat{P} \psi_{0}-\mathbf{n}_{0} \cdot \hat{L} \hat{P} \psi_{0}, \text { on } \partial_{\mathrm{Neu}} \mathcal{W}_{\alpha_{0}} .
$$

We use the Fredholm condition by taking the scalar product of the r.h.s. of (3.12) with $u_{\alpha_{0}, \eta_{0}}$ with respect to $(\hat{t}, \hat{z})$. Integrating by parts and using the Green-Riemann formula (the boundary terms cancel), this provides the equation:

$$
\mathcal{H} f_{0}=\left(\mu_{2}-\omega_{0}\right) f_{0},
$$

with:

$$
\mathcal{H}=\frac{\partial_{\eta}^{2} \nu\left(\alpha_{0}, \eta_{0}\right)}{2} D_{\hat{s}}^{2}+2 \kappa \tau_{0}^{-1}\left\|D_{\hat{z}} u_{\eta_{0}}\right\|^{2} \hat{s}^{2}
$$

and:

$$
\omega_{0}=\left\langle 2\left(\eta_{0}-\hat{t}\right) \hat{r}_{1} u_{\eta_{0}}, u_{\eta_{0}}\right\rangle_{L^{2}\left(\mathcal{S}_{\alpha}\right)}-\nu\left(\alpha_{0}, \eta_{0}\right) \int_{\mathcal{S}_{\alpha}} \frac{\hat{l}}{2} u_{\eta_{0}}^{2}+\int_{\mathcal{S}_{\alpha}} \frac{\hat{l}}{2} \hat{P} u_{\eta_{0}} \hat{P} u_{\eta_{0}}+\int_{\mathcal{S}_{\alpha}} \hat{L} \hat{P} u_{\eta_{0}} \hat{P} u_{\eta_{0}} \text {. }
$$

Up to a scaling, the $1 \mathrm{D}$-operator $\mathcal{H}$ is the harmonic oscillator on the line (we have used that Conjecture 1.9 is true). Its spectrum is given by:

$$
\left\{(2 n-1) \sqrt{\kappa \tau_{0}^{-1}\left\|D_{\hat{z}} u_{\eta_{0}}\right\|^{2} \partial_{\eta}^{2} \nu\left(\alpha_{0}, \eta_{0}\right)}, \quad n \geq 1\right\} .
$$

Therefore for $\mu_{2}$ we take:

$$
\mu_{2}=\omega_{0}+(2 n-1) \sqrt{\kappa \tau_{0}^{-1}\left\|D_{\hat{z}} u_{\eta_{0}}\right\|^{2} \partial_{\eta}^{2} \nu\left(\alpha_{0}, \eta_{0}\right)}
$$

whith $n \in \mathbb{N}^{*}$ and for $f_{0}$ the corresponding normalized eigenfunction. With this choice we deduce the existence of $\psi_{2}^{\perp}$ such that:

$$
\left(\mathcal{L}_{0}-\mu_{0}\right) \psi_{2}^{\perp}=\mu_{2} \psi_{0}-\frac{\partial_{\eta}^{2} \nu\left(\alpha_{0}, \eta_{0}\right)}{2} D_{\hat{s}}^{2} \psi_{0}-2 \kappa \tau_{0}^{-1} \hat{s}^{2} D_{\hat{z}}^{2} \psi_{0}, \text { and }\left\langle\psi_{2}^{\perp}, u_{\eta_{0}}\right\rangle_{\hat{t}, \hat{z}}=0 \text {. }
$$

We can write $\psi_{2}$ in the form:

$$
\psi_{2}=\psi_{2}^{\perp}+v_{\eta_{0}} D_{\hat{s}} f_{1}+D_{\hat{s}}^{2} f_{0} \frac{w_{\eta_{0}}}{2}+f_{2}(\hat{s}) u_{\eta_{0}},
$$

where $f_{2}$ has to be determined in a next step.

- Further terms. Let $k \geq 2$ and let us assume that we have constructed $\left(\mu_{j}\right)_{j<k+1},\left(\psi_{j}^{\perp}\right)_{j<k+1}$ and $\left(f_{j}\right)_{j<k-1}$ and that the functions $\psi_{j}$ are written in the form:

$$
\psi_{j}=\psi_{j}^{\perp}+f_{j}(\hat{s}) u_{\eta_{0}}+D_{\hat{s}} f_{j-1}(\hat{s}) v_{\eta_{0}}+D_{\hat{s}}^{2} f_{j-2} \frac{w_{\eta_{0}}}{2}
$$

with $\left\langle\psi_{j}^{\perp}, u_{\eta_{0}}\right\rangle_{\hat{t}, \hat{z}}=0$. 
We are looking for $\left(\mu_{k+1}, \psi_{k+1}^{\perp}, f_{k-1}\right)$ such that the two following equations hold:

$$
\begin{gathered}
\left(\mathcal{L}_{0}-\mu_{0}\right) \psi_{k+1}=\mu_{2} \psi_{k-1}+\mu_{k+1} \psi_{0}-\mathcal{L}_{1} \psi_{k}-\mathcal{L}_{2} \psi_{k-1}+\sum_{j=3}^{k}\left(\mu_{j}-\mathcal{L}_{j}\right) \psi_{k+1-j}, \\
\mathbf{n}_{0} \cdot \mathcal{T}_{0} \psi_{k+1}=-\mathbf{n}_{2} \mathcal{T}_{0} \psi_{k-1}-\mathbf{n}_{0} \cdot \mathcal{T}_{2} \psi_{k-1}-\sum_{\substack{a+b+c=k+1, c<k-1}} \mathbf{n}_{a} \cdot \mathcal{T}_{b} \psi_{c} .
\end{gathered}
$$

We look for $\psi_{k+1}$ in the form (see (3.16)):

$$
\psi_{k+1}=\psi_{k+1}^{\perp}+f_{k+1}(\hat{s}) u_{\eta_{0}}+D_{\hat{s}} f_{k}(\hat{s}) v_{\eta_{0}}+\frac{w_{\eta_{0}}}{2} D_{\hat{s}}^{2} f_{k-1} .
$$

Using Propositions 3.3 and 3.4, we get:

$$
\begin{aligned}
\left(\mathcal{L}_{0}-\mu_{0}\right) \psi_{k+1}=\left(\mathcal{L}_{0}-\mu_{0}\right) \psi_{k+1}^{\perp}- & 2 D_{\hat{s}} f_{k}\left(\eta_{0}-\hat{t}\right) u_{\eta_{0}} \\
& +D_{\hat{s}}^{2} f_{k-1}\left(\frac{\partial_{\eta}^{2} \nu\left(\alpha_{0}, \eta_{0}\right)}{2} u_{\eta_{0}}-2\left(\eta_{0}-\hat{t}\right) v_{\eta_{0}}-u_{\eta_{0}}\right) .
\end{aligned}
$$

The r.h.s. of (3.17) can be put in the form:

$$
\mu_{2} f_{k-1} u_{\eta_{0}}+\mu_{k+1} \psi_{0}-\mathcal{L}_{1} f_{k} u_{\eta_{0}}-\mathcal{L}_{1}\left(D_{\hat{s}} f_{k-1} v_{\eta_{0}}\right)-\mathcal{L}_{2}\left(f_{k-1} u_{\eta_{0}}\right)+R_{k}
$$

where the remainder $R_{k}$ is a determined function. Therefore (3.17) holds if and only if

$$
\begin{aligned}
\left(\mathcal{L}_{0}-\mu_{0}\right) \psi_{k+1}^{\perp}=D_{\hat{s}}^{2} f_{k-1} & \left(2\left(\hat{t}-\eta_{0}\right) v_{\eta_{0}}-u_{\eta_{0}}-\left(\frac{\partial_{\eta}^{2} \nu\left(\alpha_{0}, \eta_{0}\right)}{2} u_{\eta_{0}}-2\left(\eta_{0}-\hat{t}\right) v_{\eta_{0}}-u_{\eta_{0}}\right)\right) \\
- & \hat{s}^{2} f_{k-1}\left(2 \kappa \tau_{0}^{-1} D_{\hat{z}} u_{\eta_{0}}\right)+\left(\mu_{2} u_{\eta_{0}}-L_{2} u_{\eta_{0}}\right) f_{k-1}+\mu_{k+1} \psi_{0}+R_{k},
\end{aligned}
$$

where we have used the expressions given in (3.3) and (3.4).

Since $u_{\eta_{0}}, v_{\eta_{0}}$ and $w_{\eta_{0}}$ satisfy the Neumann boundary condition, we have $\mathbf{n}_{0} \cdot \mathcal{T}_{0} \psi_{k+1}=$ $\mathbf{n}_{0} \cdot \mathcal{T}_{0} \psi_{k+1}^{\perp}$. We take the scalar product of (3.19) with $u_{\eta_{0}}$ with respect to $(\hat{t}, \hat{z})$. Using the boundary condition (3.18) and the definition of $\omega_{0}$ (see (3.13)), we get:

$$
\left(\mathcal{H}-\left(\mu_{2}-\omega_{0}\right)\right) f_{k-1}=\mu_{k+1} f_{0}+r_{k}
$$

where $\hat{s} \mapsto r_{k}(\hat{s})$ is a known function. The compatibility condition of this 1D-equation writes

$$
\mu_{k+1}=-\left\langle r_{k}, f_{0}\right\rangle_{\check{s}}
$$

Due to the choice of $\mu_{2}$ (see (3.14)), the Fredholm alternative provides a unique function $f_{k-1}$ (orthogonal to $f_{0}$ ) which satisfies (3.20) and has the regularity and the decay properties of $r_{k}$. Using again the Fredholm alternative, we get a unique solution $\psi_{k+1}^{\perp}$ of (3.19).

- Properties of constructed functions. Let us define on $\mathcal{W}_{\alpha}$ the quasi-eigenpair:

$$
\hat{\psi}_{h}^{[J]}:=\sum_{j=0}^{J} \psi_{j} h^{j / 4} \quad \text { and } \quad \lambda^{[J]}(h):=\sum_{j=0}^{J} \mu_{j, n} h^{j / 4},
$$

where we have denoted $\mu_{j}$ by $\mu_{j, n}$ to underline the dependence on $n$ coming from (3.14). 
Due to basic Agmon's estimates the function $u_{\alpha_{0}}$ is exponentially decaying at infinity. In the $\hat{s}$ variable, the function $f_{0}$ is a gaussian and thus admits also an exponential decay. By the Fredholm alternative, all the $\psi_{j}$ have an exponential decay: there exists $h_{0}>0$ such that

$$
\exists C_{j}>0, \exists \delta>0, \forall h \in\left(0, h_{0}\right), \quad \widehat{Q}_{h}\left(e^{\delta(|\hat{s}|+|\hat{t}|+|\hat{z}|)} \psi_{j}\right) \leq C_{j}\left\|\psi_{j}\right\|_{L^{2}\left(\mathcal{W}_{\alpha}\right)}^{2}
$$

Moreover, we get the same kind of estimates in the $H^{2}$-norm. Since the infinite sector $\mathcal{S}_{\alpha}$ is a convex domain, $u_{\alpha_{0}}, v_{\alpha_{0}}$ and $w_{\alpha_{0}}$ are in $H^{2}\left(\mathcal{S}_{\alpha}\right)$, and by elliptic regularity, they belong to $C^{\infty}\left(\mathcal{S}_{\alpha}\right)$. Using the Fredholm alternative, $\hat{\psi}_{h}^{[J]}$ is in $C^{\infty} \cap H^{2}\left(\mathcal{W}_{\alpha}\right)$. Therefore all the $\hat{\psi}_{h}^{[J]}$ belong to the domain of $\widehat{Q}_{h}$ and, with (3.22), they have exponential decay. Let us recall that both $u_{\eta_{0}}$ and $f_{0}$ are normalized so that:

$$
\left\|\hat{\psi}_{h}^{[J]}\right\|_{L_{|G|^{1 / 2}}^{2}\left(\mathcal{W}_{\alpha}\right)}=1+O\left(h^{1 / 4}\right) .
$$

Nevertheless, the function $\hat{\psi}_{h}^{[J]}$ is not in the domain of $\hat{\mathcal{L}}_{h}$ (it does not satisfy the boundary condition).

- Quasimode on the lens and conclusion. Let us recall that $\mathrm{x}_{0} \in E \subset \Omega$ is the point of the edge where the opening angle is maximal. Let $\chi \in C_{0}^{\infty}(\Omega)$ which satisfies $0 \leq \chi \leq 1$, $\operatorname{supp}(\chi) \subset B\left(\mathbf{x}_{0}, 2 \epsilon_{0}\right) \cap \Omega$ and $\chi=1$ on $B\left(\mathbf{x}_{0}, \epsilon_{0}\right) \cap \Omega$. We take $\epsilon_{0}$ small enough and we use the diffeomorphism $\Psi^{-1}:(\check{s}, \check{t}, \check{z}) \mapsto \mathbf{x}$ which sends a subset of the infinite wedge to a $\epsilon_{0}$-neighborhood of $\mathbf{x}_{0} \in E$, see Section 2. Using this diffeomorphism and the scaling (3.1) we define the function on the lens:

$$
\psi_{h}^{J}(\mathbf{x}):=h^{-5 / 8} \chi(\mathbf{x}) \hat{\psi}_{h}^{[J]}\left(h^{-1 / 4} \check{s}, h^{-1 / 2} \check{t}, h^{-1 / 2} \check{z}\right) .
$$

The function $\psi_{h}^{J}$ is supported in a ball centered at $\mathbf{x}_{0}$ of radius $2 \epsilon_{0}$. Due to the factor $h^{-5 / 8}$ and to the exponential decay of $\hat{\psi}_{h}^{[J]}$, we have

$$
\left\|\psi_{h}^{J}\right\|_{L^{2}(\Omega)}=\left(1+O\left(h^{\infty}\right)\right)\left\|\hat{\psi}_{h}^{[J]}\right\|_{L^{2}\left(\mathcal{W}_{\alpha}\right)}=1+O\left(h^{1 / 4}\right) .
$$

As we have mentioned in the above, we must be careful since $\psi_{h}^{J}$ does not satisfy the Neumann type boundary condition (nevertheless it belongs to $H^{2}(\Omega)$ by construction). Let us recall that for $u \in H^{2}(\Omega)$, the Neumann trace of $u$ belongs to $H^{1 / 2}(\partial \Omega)$ with the associated Sobolev embedding. From (3.11) and the homogeneity of the norm $\|\cdot\|_{H^{1 / 2}}$, we infer:

$$
h^{1 / 4}\left\|(-i h \nabla+\mathbf{A}) \psi_{h}^{J} \cdot \mathbf{n}\right\|_{H^{1 / 2}(\partial \Omega)}=O\left(h^{(J+1) / 4}\right) .
$$

We now add a correction term to $\psi_{h}^{J}$ in order to define a quasimode which satisfies the boundary condition. We consider the following boundary value problem:

$$
\left\{\begin{aligned}
(-i h \nabla+\mathbf{A})^{2} r=0 & \text { in } \Omega, \\
(-i h \nabla+\mathbf{A}) r \cdot \mathbf{n}=g^{J} & \text { on } \partial \Omega,
\end{aligned}\right.
$$

where $g^{J}=(-i h \nabla+\mathbf{A}) \psi_{h}^{J} \cdot \mathbf{n}$. We deduce from [18] (see also [19, Theorem 5.2.1.4]) that there is a unique solution $r^{J} \in H^{2}(\Omega)$. It satisfies:

$$
h^{2}\left\|\nabla^{2} r^{J}\right\|_{L^{2}(\Omega)}+h\left\|\nabla r^{J}\right\|_{L^{2}(\Omega)}+\left\|r^{J}\right\|_{L^{2}(\Omega)} \leq C h^{1 / 2}\left\|g^{J}\right\|_{H^{1 / 2}(\partial \Omega)},
$$


with $C>0$ independent from $h$. Therefore we infer from (3.23):

$$
\left\|r^{J}\right\|_{L^{2}(\Omega)}=O\left(h^{(J+2) / 4}\right) .
$$

We can now define a quasimode on the lens:

$$
u_{h}^{J, \mathrm{qm}}:=\psi_{h}^{J}-r^{J} \text {. }
$$

It satisfies $u_{h}^{J, \mathrm{qm}} \in \operatorname{Dom}\left(\mathcal{L}_{h}\right)$ and $\left\|u_{h}^{J, q m}\right\|_{L^{2}(\Omega)}=1+O\left(h^{1 / 4}\right)$. Then, we write:

$$
\begin{aligned}
\left(\mathcal{L}_{h}-h \lambda^{[J]}(h)\right) u_{h}^{J, \mathrm{qm}} & =\left((-i h \nabla+\mathbf{A})^{2}-h \lambda^{[J]}(h)\right) u_{h}^{J, \mathrm{qm}} \\
& =(-i h \nabla+\mathbf{A})^{2} \psi_{h}^{J}-h \lambda^{[J]}(h) \psi_{h}^{J}+h \lambda^{[J]}(h) r^{J} .
\end{aligned}
$$

Thanks to Taylor expansions in the coordinates $\hat{s}, \hat{t}, \hat{z}$ and to the exponential decay of the $\psi_{j}$, we estimate by $O\left(h^{(J+1) / 4}\right)$ the term $(-i h \nabla+\mathbf{A})^{2} \psi_{h}^{J}-h \lambda^{[J]}(h) \psi_{h}^{J}$ (see (3.11)) and we deduce:

$$
\left\|\left(\mathcal{L}_{h}-h \lambda^{[J]}(h)\right) u_{h}^{J, \mathbf{q m}}\right\|_{L^{2}(\Omega)}=O\left(h^{1+(J+1) / 4}\right) .
$$

We deduce Proposition 3.1 by using the spectral theorem.

\section{Estimates of Agmon}

This section is devoted to localization estimates satisfied by the eigenfunctions. In particular we will prove that the eigenfunctions live at the scale $h^{1 / 2}$ near the edge and that they are roughly localized on the edge near $\mathbf{x}_{0}$. For that purpose we will use the "standard" estimates of Agmon.

4.1. A first rough estimate for the eigenvalues. Let us begin by proving a rough and standard estimate for the eigenvalues.

Proposition 4.1. There exist $C$ and $h_{0}>0$ such that, for $h \in\left(0, h_{0}\right)$ :

$$
\lambda_{n}(h) \geq \nu\left(\alpha_{0}\right) h-C h^{5 / 4} .
$$

Proof. Let us introduce a partition of unity $\left(\chi_{j, h}\right)_{j \geq 0}$ with balls of centers $\mathbf{x}_{j}$ and of size $h^{\rho}$ where $\rho>0$ will be chosen later:

$$
\sum_{j} \chi_{j, h}^{2}=1, \quad \text { on } \Omega
$$

and such that:

$$
\sum_{j}\left\|\nabla \chi_{j, h}\right\|^{2} \leq C h^{-2 \rho}
$$

The "IMS" formula (see [11]) provides the following decomposition for the quadratic form:

$$
\mathcal{Q}_{h}(\psi)=\sum_{j}\left(\mathcal{Q}_{h}\left(\chi_{j, h} \psi\right)-h^{2}\left\|\nabla \chi_{j, h} \psi\right\|^{2}\right)
$$

so that:

$$
\mathcal{Q}_{h}(\psi) \geq \sum_{j} \mathcal{Q}_{h}\left(\chi_{j, h} \psi\right)-C h^{2-2 \rho}\|\psi\|^{2} .
$$


Let us now distinguish between three kinds of balls.

$$
\begin{aligned}
& J_{1}(h)=\left\{j: \operatorname{supp}\left(\chi_{j, h}\right) \cap \partial \Omega=\emptyset\right\}, \\
& J_{2}(h)=\left\{j: \operatorname{supp}\left(\chi_{j, h}\right) \cap \partial \Omega \neq \emptyset \text { and } \operatorname{supp}\left(\chi_{j, h}\right) \cap E=\emptyset\right\}, \\
& J_{3}(h)=\left\{j: \operatorname{supp}\left(\chi_{j, h}\right) \cap E \neq \emptyset\right\} .
\end{aligned}
$$

- Case when $j \in J_{1}(h)$ : interior case. We have:

$$
\mathcal{Q}_{h}\left(\chi_{j, h} \psi\right) \geq h\left\|\chi_{j, h} \psi\right\|^{2} \geq h \nu\left(\alpha_{0}\right)\left\|\chi_{j, h} \psi\right\|^{2} .
$$

- Case when $j \in J_{2}(h)$ : regular boundary case. Let us now consider the boundary balls which do not cross the edge. We can assume that such balls have their centers on the boundary and we denote by $\theta_{j}$ the angle between the magnetic field $\boldsymbol{\beta}$ and the boundary at the point $\mathbf{x}_{j}$. There exist local coordinates $\Phi\left(y_{1}, y_{2}, y_{3}\right)=\mathbf{x}$ so that the derivative satisfies $D \Phi=I d+$ $O\left(h^{\rho}\right)$ and:

$$
\begin{aligned}
& \mathcal{Q}_{h}\left(\chi_{j, h} \psi\right) \\
& \geq\left(1-C h^{\rho}\right) \int_{y_{3}>0}\left|\left(h D_{y_{1}}+\tilde{A}_{1}\right) \widetilde{\chi_{j, h} \psi}\right|^{2}+\left|\left(h D_{y_{2}}+\tilde{A}_{2}\right) \widetilde{\chi_{j, h} \psi}\right|^{2}+\left|\left(h D_{y_{3}}+\tilde{A}_{3}\right) \widetilde{\chi_{j, h} \psi}\right|^{2} d y,
\end{aligned}
$$

where the tilde means that the functions are considered in the new coordinates. In particular, we have $\tilde{\mathbf{A}}=D \Phi \mathbf{A}(\Phi)$. We can introduce the linear approximation of $\tilde{\mathbf{A}}$ denoted by $\tilde{\mathbf{A}}^{\text {lin }}$ :

$$
\left\|\tilde{\mathbf{A}}-\tilde{\mathbf{A}}^{\operatorname{lin}}\right\| \leq C h^{2 \rho}
$$

For all $\varepsilon \in(0,1)$, we get $^{1}$ :

$$
\begin{aligned}
& \mathcal{Q}_{h}\left(\chi_{j, h} \psi\right) \geq \\
& (1-\varepsilon)\left(1-C h^{\rho}\right) \int_{y_{3}>0}\left|\left(h D_{y_{1}}+\tilde{A}_{1}^{\text {lin }}\right) \widetilde{\chi_{j, h} \psi}\right|^{2}+\left|\left(h D_{y_{2}}+\tilde{A}_{2}^{\text {lin }}\right) \widetilde{\chi_{j, h} \psi}\right|^{2}+\left|\left(h D_{y_{3}}+\tilde{A}_{3}^{\text {lin }}\right) \widetilde{\chi_{j, h} \psi}\right|^{2} d y \\
& -C \varepsilon^{-1} h^{4 \rho}\left\|\widetilde{\chi_{j, h} \psi}\right\|^{2} \text {. }
\end{aligned}
$$

Therefore we are reduced to the magnetic Laplacian in the half-space $\mathbb{R}_{+}^{3}$ with a constant magnetic field making an angle $\theta_{j}$ with the boundary (see Section 1.2.2) and we can use the lower bound:

$$
\mathcal{Q}_{h}\left(\chi_{j, h} \psi\right) \geq(1-\varepsilon)\left(1-C h^{\rho}\right) h \sigma\left(\theta_{j}\right) \widetilde{\left\|\widetilde{\chi_{j, h} \psi}\right\|^{2}}-C \varepsilon^{-1} h^{4 \rho}\left\|\widetilde{\chi_{j, h} \psi}\right\|^{2} .
$$

We use the inequality of Assumption 1.12 and we take $\varepsilon=h^{2 \rho-1 / 2}$ to get:

$$
\mathcal{Q}_{h}\left(\chi_{j, h} \psi\right) \geq h \nu\left(\alpha_{0}\right)\left\|\chi_{j, h} \psi\right\|^{2}-C h^{2 \rho+1 / 2}\left\|\chi_{j, h} \psi\right\|^{2}-C h^{1+\rho}\left\|\chi_{j, h} \psi\right\|^{2} .
$$

\footnotetext{
${ }^{1}$ We have used the standard inequality $|a+b|^{2} \geq(1-\varepsilon)|a|^{2}-\varepsilon^{-1}|b|^{2}$.
} 
- Case when $j \in J_{3}(h)$. Let us consider the boundary balls which cross the edge $E$. We can assume that such balls have their centers on $E$. We can use the local coordinates of Section 2: $\Psi(\check{s}, \check{t}, \check{z})=\mathbf{x}$, which transform the edge with variable angle into the wedge with constant angle $\alpha_{j}: \mathcal{W}_{\alpha_{j}}$. Firstly we erase the curvature terms by writing:

$$
\mathcal{Q}_{h}\left(\chi_{j, h} \psi\right) \geq\left(1-C h^{\rho}\right) \int\left\|\check{\nabla}_{h}\left(\check{\chi}_{j, h} \check{\psi}\right)\right\|^{2} d \check{s} d \check{t} d \check{z} .
$$

We have:

$$
\begin{aligned}
& \left\|\check{\nabla}_{h}\left(\check{\chi}_{j, h} \check{\psi}\right)\right\|^{2} \\
& \geq(1-\varepsilon)\left(\left\|\left(h D_{\check{s}}+\eta_{0}-\check{t}\right)\left(\check{\chi}_{j, h} \check{\psi}\right)\right\|^{2}+h^{2}\left\|D_{\check{t}}\left(\check{\chi}_{j, h} \check{\psi}\right)\right\|^{2}+h^{2}\left\|D_{\check{z}}\left(\check{\chi}_{j, h} \check{\psi}\right)\right\|^{2}\right) \\
& -\varepsilon^{-1}\left(C\left(h^{4 \rho}+h^{2}\right)\left\|\check{\chi}_{j, h} \check{\psi}\right\|^{2}+C h^{2 \rho}\left\|h D_{\check{z}}\left(\check{\chi}_{j, h} \check{\psi}\right)\right\|^{2}\right)
\end{aligned}
$$

We choose $\varepsilon=h^{2 \rho-1 / 2}$. We deduce:

$$
\mathcal{Q}_{h}\left(\chi_{j, h} \psi\right) \geq\left(\nu\left(\alpha_{j}\right) h-C h^{1 / 2+2 \rho}-C h^{1+\rho}-C h^{5 / 2-2 \rho}\right)\left\|\chi_{j, h} \psi\right\|^{2} .
$$

In order to optimize the different remainders here and in (4.3), we are led to choose $\rho$ such that $2-2 \rho=1 / 2+2 \rho$, that is $\rho=3 / 8$ and the conclusion follows.

4.2. Normal estimates of Agmon. From the rough control of the eigenvalues, we deduce the standard "normal estimates of Agmon".

Proposition 4.2. There exist $\varepsilon_{0}>0, h_{0}>0$ and $C>0$ such that for all $h \in\left(0, h_{0}\right)$ we have the following estimates for an eigenfunction $\psi_{n}$ associated to $\lambda_{n}(h)$ :

$$
\begin{aligned}
& \int e^{2 \varepsilon_{0} h^{-1 / 2} d(\mathbf{x}, E)}\left|\psi_{n}\right|^{2} d \mathbf{x} \leq C\left\|\psi_{n}\right\|^{2}, \\
& \mathcal{Q}_{h}\left(e^{\varepsilon_{0} h^{-1 / 2} d(\mathbf{x}, E)} \psi_{n}\right) \leq C h\left\|\psi_{n}\right\|^{2} .
\end{aligned}
$$

Proof. We use a partition of unity $\left(\chi_{j, h}\right)_{j \geq 0}$ with balls of size $R h^{1 / 2}$ and we let $\Phi(\mathbf{x})=$ $\varepsilon_{0} h^{-1 / 2} d(\mathbf{x}, E)$. We write the identity of Agmon:

$$
\lambda_{n}(h)\left\|e^{\Phi} \psi_{n}\right\|^{2}=\mathcal{Q}_{h}\left(e^{\Phi} \psi_{n}\right)-h^{2}\left\|\nabla \Phi e^{\Phi} \psi_{n}\right\|^{2} .
$$

We deduce the inequality:

$$
\sum_{j}\left(\mathcal{Q}_{h}\left(\chi_{j, h} e^{\Phi} \psi_{n}\right)-\lambda_{n}(h)\left\|\chi_{j, h} e^{\Phi} \psi_{n}\right\|^{2}-h\left(\varepsilon_{0}^{2}+\frac{C_{0}}{R^{2}}\right)\left\|\chi_{j, h} e^{\Phi} \psi_{n}\right\|^{2}\right) \leq 0 .
$$

We split the sum into three parts as in the proof of Proposition 4.1 and we use in the same way changes of variables, taking into account that the ball are now of size $R h^{1 / 2}$. We recall that, thanks to Proposition 3.1, we have:

$$
\lambda_{n}(h) \leq \nu\left(\alpha_{0}\right) h+C h^{3 / 2} .
$$

- Case when $j \in J_{1}(h)$ : interior case. We have:

$$
\mathcal{Q}_{h}\left(\chi_{j, h} e^{\Phi} \psi_{n}\right) \geq h\left\|\chi_{j, h} e^{\Phi} \psi_{n}\right\|^{2} \geq h \nu\left(\alpha_{0}\right)\left\|\chi_{j, h} e^{\Phi} \psi_{n}\right\|^{2} .
$$


- Case when $j \in J_{2}(h)$ : regular boundary case. We have:

$$
\mathcal{Q}_{h}\left(\chi_{j, h} e^{\Phi} \psi_{n}\right) \geq \sigma\left(\theta_{j}\right) h\left\|\chi_{j, h} e^{\Phi} \psi_{n}\right\|^{2}-C h^{3 / 2}\left\|\chi_{j, h} e^{\Phi} \psi_{n}\right\|^{2} .
$$

- Case when $j \in J_{3}(h)$. We have:

$$
\mathcal{Q}_{h}\left(\chi_{j, h} e^{\Phi} \psi_{n}\right) \geq\left(\nu\left(\alpha_{j}\right) h-C h^{3 / 2}\right)\left\|\chi_{j, h} e^{\Phi} \psi_{n}\right\|^{2} .
$$

Therefore we get, from (4.9), the existence of $C>0$ and $h_{0}>0$ such that for $h_{0} \in(0, h)$ :

$$
\begin{aligned}
h\left(\inf _{j} \sigma\left(\theta_{j}\right)-\nu\left(\alpha_{0}\right)-\left(\epsilon_{0}^{2}+\frac{C_{0}}{R^{2}}\right)-C h^{1 / 2}\right) \sum_{J_{1} \cup J_{2}} & \left\|\chi_{j, h} e^{\Phi} \psi_{n}\right\|^{2} \\
& \leq h\left(\epsilon_{0}^{2}+\frac{C_{0}}{R^{2}}+C h^{1 / 2}\right) \sum_{J_{3}}\left\|\chi_{j, h} e^{\Phi} \psi_{n}\right\|^{2}
\end{aligned}
$$

Using (1.15) we get that for $\epsilon_{0}$ small enough and $R$ large enough, there exist $h_{0}>0, c>0$ and $\tilde{C}>0$ such that for $h \in\left(0, h_{0}\right)$ we have:

$$
c<\left(\inf _{j} \sigma\left(\theta_{j}\right)-\nu\left(\alpha_{0}\right)-\epsilon_{0}^{2}-\frac{C_{0}}{R^{2}}-C h^{1 / 2}\right) \quad \text { and } \quad\left(\epsilon_{0}^{2}+\frac{C_{0}}{R^{2}}+C h^{1 / 2}\right) \leq \tilde{C} .
$$

We deduce that for $h \in\left(0, h_{0}\right)$ :

$$
c \sum_{J_{1} \cup J_{2}}\left\|\chi_{j, h} e^{\Phi} \psi_{n}\right\|^{2} \leq \tilde{C} \sum_{J_{3}}\left\|\chi_{j, h} e^{\Phi} \psi_{n}\right\|^{2} \leq \tilde{C} e^{2 \epsilon_{0} R} \sum_{J_{3}}\left\|\chi_{j, h} \psi_{n}\right\|^{2} \leq C_{1}\left\|\psi_{n}\right\|^{2} .
$$

Therefore (4.6) follows and we get (4.7) from the identity (4.8).

4.3. Rough tangential estimates. We can also use the rough estimate of the eigenvalues to deduce a rough localization of the eigenfunctions near $\mathbf{x}_{0}$.

Proposition 4.3. There exist $h_{0}>0$ and $C>0$ such that for all $h \in\left(0, h_{0}\right)$, we have the following estimates for an eigenfunction $\psi_{n}$ associated to $\lambda_{n}(h)$ :

$$
\begin{aligned}
& \int e^{\chi(\mathbf{x}) h^{-1 / 8}|s(\mathbf{x})|}\left|\psi_{n}\right|^{2} d \mathbf{x} \leq C\left\|\psi_{n}\right\|^{2}, \\
& \mathcal{Q}_{h}\left(e^{\chi(\mathbf{x}) h^{-1 / 8}|s(\mathbf{x})|} \psi_{n}\right) \leq C h\left\|\psi_{n}\right\|^{2},
\end{aligned}
$$

where $\chi$ is a smooth cutoff function supported in a fixed neighborhood of $E$.

Proof. We use a partition of unity $\left(\chi_{j, h}\right)_{j \geq 0}$ with balls of size $h^{3 / 8}$ as in the proof of Proposition 4.1. We let $\Phi=\chi(\mathbf{x}) h^{-1 / 8}|s(\mathbf{x})|$ where the tangential coordinate $s$ is defined in Section 2 . In particular, we have $\|\nabla \Phi\|^{2} \leq C_{0} h^{-1 / 4}$ and $\left\|\nabla \chi_{j, h}\right\|^{2} \leq \widetilde{C}_{0} h^{-3 / 4}$. As in the proof of the Proposition 4.2, we combine (4.8) and (4.2), we use a change of variables and the min-max principle for the sum over $J_{1} \cup J_{2}$ and we get $C_{1}>0$ such that

$$
\begin{aligned}
\left(h \inf _{j} \sigma\left(\theta_{j}\right)-\lambda_{n}(h)-C_{1} h^{5 / 4}\right) & \sum_{j \in J_{1}(h) \cup J_{2}(h)}\left\|\chi_{j, h} e^{\Phi} \psi_{n}\right\|^{2} \\
& +\sum_{j \in J_{3}(h)} \mathcal{Q}_{h}\left(\chi_{j, h} e^{\Phi} \psi_{n}\right)-\left(\lambda_{n}(h)+C_{1} h^{5 / 4}\right)\left\|\chi_{j, h} e^{\Phi} \psi_{n}\right\|^{2} \leq 0 .
\end{aligned}
$$


Using Assumption 1.12 and (4.10), we get $h_{0}>0$ and $c>0$ such that for $h \in\left(0, h_{0}\right)$ we have $\left(h \inf _{j} \sigma\left(\theta_{j}\right)-\lambda_{n}(h)-C_{1} h^{5 / 4}\right) \geq c h$. We make a partition of $J_{3}$ depending on how far we are from the point $\mathbf{x}_{0}$ where $\alpha$ is maximal, we get:

$$
\begin{gathered}
c h \sum_{j \in J_{1}(h) \cup J_{2}(h)}\left\|\chi_{j, h} e^{\Phi} \psi_{n}\right\|^{2}+\sum_{\substack{j \in J_{3}(h) \\
\left\|\mathbf{x}_{j}-\mathbf{x}_{0}\right\| \geq \delta_{0} h^{1 / 8}}}\left(\left(\nu\left(\alpha_{j}\right)-\nu\left(\alpha_{0}\right)\right) h-C_{1} h^{5 / 4}\right)\left\|\chi_{j, h} e^{\Phi} \psi_{n}\right\|^{2} \\
\leq \sum_{\substack{j \in J_{3}(h) \\
\left\|\mathbf{x}_{j}-\mathbf{x}_{0}\right\| \leq \delta_{0} h^{1 / 8}}}-\mathcal{Q}_{h}\left(\chi_{j, h} e^{\Phi} \psi_{n}\right)+\left(\nu\left(\alpha_{0}\right) h+C_{1} h^{5 / 4}\right)\left\|\chi_{j, h} e^{\Phi} \psi_{n}\right\|^{2}
\end{gathered}
$$

We have:

$$
-\mathcal{Q}_{h}\left(\chi_{j, h} e^{\phi} \psi_{n}\right)+\left(\nu\left(\alpha_{0}\right) h+C_{1} h^{5 / 4}\right)\left\|\chi_{j, h} e^{\Phi} \psi_{n}\right\|^{2} \leq \tilde{C} h^{5 / 4}\left\|\chi_{j, h} e^{\Phi} \psi_{n}\right\|^{2} .
$$

We write $\left\{\left\|\mathbf{x}_{j}-\mathbf{x}_{0}\right\| \geq \delta_{0} h^{1 / 8}\right\}=\left\{\left\|\mathbf{x}_{j}-\mathbf{x}_{0}\right\| \geq r_{0}\right\} \cup\left\{\delta_{0} h^{1 / 8} \leq\left\|\mathbf{x}_{j}-\mathbf{x}_{0}\right\| \leq r_{0}\right\}$ and we have:

- If $\delta_{0} h^{1 / 8} \leq\left\|\mathbf{x}_{j}-\mathbf{x}_{0}\right\| \leq r_{0}$, we use the non-degeneracy of $\alpha$ near $x_{0}$ (we have assumed Assumption 1.14): for $r_{0}$ small enough we have (Conjecture 1.9 is assumed to be true):

$$
\nu\left(\alpha_{j}\right)-\nu\left(\alpha\left(s_{0}\right)\right) \geq \frac{\nu^{\prime \prime}\left(\alpha\left(s_{0}\right)\right)}{4}\left(s_{j}-s_{0}\right)^{2} \geq \frac{\nu^{\prime \prime}\left(\alpha\left(s_{0}\right)\right)}{4} \delta_{0}^{2} h^{1 / 4} .
$$

We choose $\delta_{0}$ such that:

$$
\frac{\nu^{\prime \prime}\left(\alpha\left(s_{0}\right)\right)}{4} \delta_{0}^{2}>C_{1}
$$

and we deduce:

$$
\forall h \in\left(0, h_{0}\right), \quad\left(\nu\left(\alpha_{j}\right)-\nu\left(\alpha_{0}\right)\right) h-C_{1} h^{5 / 4} \geq c h^{5 / 4}
$$

- If $\left\|\mathbf{x}_{j}-\mathbf{x}_{0}\right\| \geq r_{0}$, due to the uniqueness of the maximum of $s \mapsto \alpha(s)$, there exists $\eta>0$ such that $\nu\left(\alpha_{j}\right)-\nu\left(\alpha_{0}\right) \geq \eta$ and therefore we get the existence of $c>0$ such that:

$$
\forall h \in\left(0, h_{0}\right), \quad\left(\nu\left(\alpha_{j}\right)-\nu\left(\alpha_{0}\right)\right) h-C_{1} h^{5 / 4} \geq c h
$$

Using (4.12), (4.13) and (4.14) into (4.11) we get:

$$
\begin{gathered}
c h \sum_{j \in J_{1}(h) \cup J_{2}(h)}\left\|\chi_{j, h} e^{\Phi} \psi_{n}\right\|^{2}+c h \sum_{\substack{j \in J_{3}(h) \\
\left\|\mathbf{x}_{j}-\mathbf{x}_{0}\right\| \geq r_{0}}}\left\|\chi_{j, h} e^{\Phi} \psi_{n}\right\|^{2}+c h^{5 / 4} \sum_{\substack{j \in J_{3}(h) \\
\delta_{0} h^{1 / 8} \leq\left\|\mathbf{x}_{j}-\mathbf{x}_{0}\right\| \leq r_{0}}}\left\|\chi_{j, h} e^{\Phi} \psi_{n}\right\|^{2} \\
\leq C h^{5 / 4} \sum_{\substack{j \in J_{3}(h) \\
\left\|\mathbf{x}_{j}-\mathbf{x}_{0}\right\| \leq \delta_{0} h^{1 / 8}}}\left\|\chi_{j, h} e^{\Phi} \psi_{n}\right\|^{2} .
\end{gathered}
$$

Using the fact that $\Phi$ is bounded on $\left\{\left\|\mathbf{x}_{j}-\mathbf{x}_{0}\right\| \leq \delta_{0} h^{1 / 8}\right\}$, we conclude in the same way as in the proof of Proposition 4.2.

We use a cutoff function $\chi_{h}(\mathbf{x})$ near $\mathbf{x}_{0}$ such that:

$$
\chi_{h}(\mathbf{x})=\chi_{0}\left(h^{-1 / 8+\gamma} \check{s}(\mathbf{x})\right) \chi_{0}\left(h^{-1 / 2+\gamma} \check{t}(\mathbf{x})\right) \chi_{0}\left(h^{-1 / 2+\gamma} \check{z}(\mathbf{x})\right) \quad \text { with } 0<\gamma<\frac{1}{8} .
$$


- Space of the eigenfunctions. For all $N \geq 1$, let us consider $L^{2}$-normalized eigenpairs $\left(\lambda_{n}(h), \psi_{n}\right)_{1 \leq n \leq N}$ such that $\left\langle\psi_{n}, \psi_{m}\right\rangle=0$ when $n \neq m$. We consider the $N$ dimensional space defined by:

$$
\check{\mathfrak{E}}_{N}(h)=\operatorname{span}_{1 \leq n \leq N} \check{\psi}_{n}, \quad \text { where } \quad \check{\psi}_{n}=\chi_{h} \psi_{n} .
$$

Notation 4.4. We will denote by $\check{\psi}\left(=\chi_{h} \psi\right)$ the elements of $\check{\mathfrak{E}}_{N}(h)$.

Corollary 4.5. Let $\psi \in \operatorname{span}\left(\psi_{n}\right)_{1 \leq n \leq N}$ and $\check{\psi}=\chi_{h} \psi$. Then we have:

$$
\begin{array}{rll}
\|\psi\|=\|\check{\psi}\|\left(1+O\left(h^{\infty}\right)\right) & \text { and } & \check{\mathcal{Q}}_{h}(\psi)=\check{\mathcal{Q}}_{h}(\check{\psi})\left(1+O\left(h^{\infty}\right)\right), \\
\left\|D_{\check{s}} \psi\right\|=\left\|D_{\check{s}} \check{\psi}\right\|\left(1+O\left(h^{\infty}\right)\right) & \text { and } & \check{\mathcal{Q}}_{h}\left(D_{j} \psi\right)=\check{\mathcal{Q}}_{h}\left(D_{j} \check{\psi}\right)\left(1+O\left(h^{\infty}\right)\right),
\end{array}
$$

where $j=\check{s}, \check{t}, \check{z}$. Moreover, we have:

$$
\check{\mathcal{Q}}_{h}\left(D_{\check{s}}\left(\underline{\chi_{h}} \psi\right)=O\left(h^{\infty}\right)\|\psi\|^{2}\right.
$$

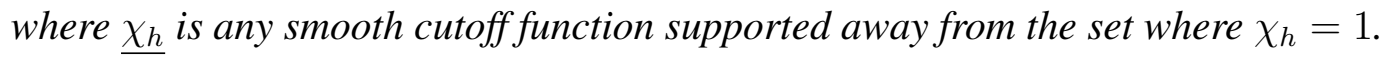

Proof. The first line comes directly from Propositions 4.2 and 4.3. In order to get the estimates of order two on $\psi$ (note that these estimates have a meaning since the eigenfunctions are in $H^{2}$ ), we use the elliptic technics of [21] (see also [37, Proposition 2.6]).

Proposition 4.6. There exist $h_{0}>0, C>0$ such that for $h \in\left(0, h_{0}\right)$ and for all $\breve{\psi} \in \check{\mathfrak{E}}_{N}(h)$ we have for all $k$ and $l$ in $\mathbb{N}$ :

$$
\int|\check{t}|^{k}|\check{z}|^{l}|\check{\psi}|^{2} d \check{s} d \check{t} d \check{z} \leq C h^{k / 2} h^{l / 2}\|\check{\psi}\|^{2}
$$

$$
\int|\check{t}|^{k}|\check{z}|^{l}\left(\left|h D_{\check{t}} \check{\psi}\right|^{2}+\left|h D_{\check{z}} \check{\psi}\right|^{2}+\left|\left(h D_{\check{s}}-\check{t}+h^{1 / 2} \eta_{0}\right) \check{\psi}\right|^{2}\right) d \check{s} d \check{t} d \check{z} \leq C h h^{k / 2} h^{l / 2}\|\check{\psi}\|^{2} .
$$

Proof. For any eigenfunction $\psi$, we have due to Proposition 4.2:

$$
\int e^{2 \varepsilon_{0} d(\mathbf{x}, E) h^{-1 / 2}}\left|\chi_{h} \psi\right|^{2} d \mathbf{x} \leq C\|\psi\|^{2} \leq \tilde{C}\left\|\chi_{h} \psi\right\|^{2}
$$

For all $n \geq 1$, we have:

$$
\int d(\mathbf{x}, E)^{n}\left|\chi_{h} \psi\right|^{2} d \mathbf{x} \leq C(n) h^{n / 2}\left\|\chi_{h} \psi\right\|^{2}
$$

Using the coordinates $(\check{s}, \check{t}, \check{z})$ on the support of $\chi_{h}$, we infer:

$$
\int(|\check{t}|+|\check{z}|)^{n}\left|\chi_{h} \psi\right|^{2} d \mathbf{x} \leq \tilde{C}(n) h^{n / 2}\left\|\chi_{h} \psi\right\|^{2}
$$

and we deduce (4.19). We get (4.20) in a similar way by using (4.7).

Proposition 4.7. For all $n \geq 1$, there exists $h_{0}>0$ such that for $h \in\left(0, h_{0}\right)$, we have:

$$
\lambda_{n}(h)=\nu\left(\alpha_{0}, \eta_{0}\right) h+O\left(h^{3 / 2}\right) .
$$


Proof. We have:

$$
\check{\mathcal{Q}}_{h}(\check{\psi})=\left\langle\check{G}^{-1} \check{\nabla}_{h} \check{\psi}, \check{\nabla}_{h} \check{\psi}\right\rangle_{L_{|G|^{2} / 2}(d \check{s} d \check{t} d \check{z})}
$$

With the Taylor expansion of $\check{G}^{-1}$ and $|\check{G}|$ (see (2.3), (2.4) and (2.5)) and the estimates of Agmon with respect to $\check{t}$ and $\check{z}$ (see Proposition 4.2), we infer:

$$
\check{\mathcal{Q}}_{h}(\check{\psi}) \geq \check{\mathcal{Q}}_{h}^{\text {flat }}(\check{\psi})-C h^{3 / 2}\|\check{\psi}\|^{2} \text {. }
$$

where $\check{\mathcal{Q}}_{h}^{\text {flat }}$ is the quadratic form associated to the leading operator in the $(\check{s}, \check{t}, \check{z})$ coordinates:

$$
\check{\mathcal{Q}}_{h}^{\text {flat }}(\check{\psi})=\left\|h D_{\check{t}} \check{\psi}\right\|^{2}+\left\|h \tau_{0} \tau(\check{s})^{-1} D_{\check{z}} \check{\psi}\right\|^{2}+\left\|\left(h D_{\check{s}}+\eta_{0} h^{1 / 2}-\check{t}\right) \check{\psi}\right\|^{2} .
$$

Moreover, we have:

$$
\check{\mathcal{Q}}_{h}^{\text {flat }}(\check{\psi}) \geq\left\|h D_{\check{t}} \check{\psi}\right\|^{2}+\left\|h D_{\check{z}} \check{\psi}\right\|^{2}+\left\|\left(h D_{\check{s}}+\eta_{0} h^{1 / 2}-\check{t}\right) \check{\psi}\right\|^{2} \geq \nu\left(\alpha_{0}, \eta_{0}\right) h\|\check{\psi}\|^{2} .
$$

It remains to use Proposition 3.1 and the conclusion follows.

\section{LOCAL AND MICROLOCAL ESTIMATES}

As we have seen in Section 3 (see (3.2)), we can think that $h^{2} D_{\check{t}}^{2}+h^{2} D_{\check{z}}^{2}+\left(\eta_{0} h^{1 / 2}-\check{t}\right)^{2}$ is an approximation of $\mathcal{L}_{h}$ when acting on the eigenfunctions. This property is not obvious and relies on the behavior of the tangential derivative $D_{\breve{s}}$ when acting on the eigenfunctions. Let us also notice that the localization techniques "à la Agmon" are not enough to establish the fine localization properties (with respect to $\check{s}$ ) satisfied by the eigenfunctions.

5.1. Control of $D_{\check{s}}$. We will need three lemmas to get an optimal control of the eigenfunctions with respect to $D_{\check{s}}$. We recall that $\check{\mathcal{L}}_{h}=|\check{G}|^{-1 / 2} \check{\nabla}_{h}|\check{G}|^{1 / 2} \check{G}^{-1} \check{\nabla}_{h}$ and that $\check{\nabla}_{h}$ is defined in (1.17). We denote $\check{g}=|\check{G}|^{1 / 2} \check{G}^{-1}$ so that, for $\psi \in \operatorname{Dom}\left(\check{\mathcal{Q}}_{h}\right)$, we have $\check{\mathcal{Q}}_{h}(\psi)=$ $\int \check{g}\left|\check{\nabla}_{h} \psi\right|^{2} d \check{s} d \check{t} d \check{z}$. If we denote $\check{\nabla}_{h}=\left(P_{1}, P_{2}, P_{3}\right)$, we notice:

$$
\check{\mathcal{Q}}_{h}(\psi)=\int_{\mathcal{W}_{\alpha}} \check{g} \sum_{j=1}^{3}\left|P_{j} \psi\right|^{2} d \check{s} d \check{t} d \check{z},
$$

which reminds a form "à la Hörmander" (see [26, Theorem 18.5.9 and below]). Therefore we can expect a generalized "IMS" formula for the quantity $\left\langle\breve{\mathcal{L}}_{h} \psi, \psi\right\rangle_{L_{|\breve{G}|^{1 / 2}}^{2}}$ :

Lemma 5.1. For an eigenpair $(\lambda, \psi)$ such that $\lambda$ satisfies $\lambda \leq \nu\left(\alpha_{0}\right) h+C h^{3 / 2}$ (see also (4.10)), we have the commutator formula:

$$
\begin{aligned}
& \left(\lambda+O\left(h^{\infty}\right)\right)\left\|D_{\check{s}} \check{\psi}\right\|_{L_{|\check{G}|^{1 / 2}}^{2}}^{2}+O\left(h^{\infty}\right)\|\check{\psi}\|_{L_{|\check{G}|^{1 / 2}}^{2}}^{2} \\
& =\check{\mathcal{Q}}_{h}\left(D_{\check{s}} \check{\psi}\right)-\left\langle\check{g}\left[\check{\nabla}_{h}, D_{\check{s}}\right] \check{\psi},\left[\check{\nabla}_{h}, D_{\check{s}}\right] \check{\psi}\right\rangle+\Re\left(\left\langle\check{g} \check{\nabla}_{h} \check{\psi},\left[\left[\check{\nabla}_{h}, D_{\check{s}}\right], D_{\check{s}}\right] \breve{\psi}\right\rangle\right) \\
& +\Re\left(\left\langle\left(D_{\breve{s}} \check{g}\right) \check{\nabla}_{h} \check{\psi}, \check{\nabla}_{h} D_{\breve{s}} \check{\psi}\right\rangle+\left\langle\left(D_{\check{s}} \check{g}\right) \check{\nabla}_{h} \check{\psi},\left[\check{\nabla}_{h}, D_{\breve{s}}\right] \check{\psi}\right\rangle+\lambda\left\langle\left(D_{\check{s}}|\check{G}|^{1 / 2}\right) \check{\psi}, D_{\breve{s}} \check{\psi}\right\rangle\right) \text {. }
\end{aligned}
$$

where:

$$
\check{g}=|\check{G}|^{1 / 2} \check{G}^{-1}
$$

and where $\check{\psi}=\chi_{h} \psi$ with $\chi_{h}$ defined in (4.15). 
Proof. We shorten the notation by using $L_{|\check{G}|^{1 / 2}}^{2}=L^{2}\left(|\check{G}|^{1 / 2}, d \check{s} d \check{t} d \check{z}\right)$ and $\langle\cdot, \cdot\rangle_{L_{|\check{G}|^{1 / 2}}^{2}}$ to denote the associated scalar product. The function $\check{\psi}$ does not satisfy the boundary condition associated to $\check{\mathcal{L}}$ but it is in $H^{2}$ so that the following formula makes sense ( $\check{\mathcal{L}}$ is considered as a partial differential operator):

$$
\begin{aligned}
\left\langle\check{\mathcal{L}}_{h} \check{\psi}, D_{\breve{s}}^{2} \check{\psi}\right\rangle_{L_{|\check{G}|^{1 / 2}}^{2}} & =\left\langle\chi_{h} \check{\mathcal{L}}_{h} \psi, D_{\breve{s}}^{2} \check{\psi}\right\rangle_{L_{|\check{G}|^{1 / 2}}^{2}}+\left\langle\left[\check{\mathcal{L}}_{h}, \chi_{h}\right] \psi, D_{\check{s}}^{2} \check{\psi}\right\rangle_{L_{|\check{G}|^{1 / 2}}^{2}} \\
& =\left\langle\lambda \check{\psi}, D_{\breve{s}}^{2} \check{\psi}\right\rangle_{L_{|\check{G}|^{1 / 2}}^{2}}+\left\langle\left[\check{\mathcal{L}}_{h}, \chi_{h}\right] \psi, D_{\check{s}}^{2} \check{\psi}\right\rangle_{L_{|\check{G}|^{1 / 2}}^{2}} .
\end{aligned}
$$

An integration by parts jointly with Corollary 4.5 (especially (4.18)) implies:

$$
\left\langle\left[\check{\mathcal{L}}_{h}, \chi_{h}\right] \psi, D_{\check{s}}^{2} \check{\psi}\right\rangle_{L_{|\check{G}|^{1 / 2}}^{2}}=O\left(h^{\infty}\right)\|\psi\|_{L_{|\check{G}|^{1 / 2}}^{2}}^{2},
$$

indeed $\left[\check{\mathcal{L}}_{h}, \chi_{h}\right] \psi$ is supported away from the set where $\chi_{h}=1$. Another integration by parts provides:

$$
\left\langle\check{\psi}, D_{\breve{s}}^{2} \check{\psi}\right\rangle_{L_{|\check{G}|^{1 / 2}}^{2}}=\left\|D_{\check{s}} \check{\psi}\right\|_{L_{|\check{G}|^{1 / 2}}^{2}}^{2}+\left\langle\left(D_{\breve{s}}|\check{G}|^{1 / 2}\right) \check{\psi}, D_{\check{s}} \check{\psi}\right\rangle
$$

We deduce that:

$$
\left\langle\check{\mathcal{L}}_{h} \check{\psi}, D_{\check{s}}^{2} \check{\psi}\right\rangle_{L_{|\breve{G}|^{1 / 2}}^{2}}=\left(\lambda+O\left(h^{\infty}\right)\right)\left\|D_{\check{s}} \check{\psi}\right\|_{L_{|\breve{G}|^{1 / 2}}^{2}}^{2}+\lambda\left\langle\left(D_{\breve{s}}|\check{G}|^{1 / 2}\right) \check{\psi}, D_{\check{s}} \check{\psi}\right\rangle+O\left(h^{\infty}\right)\|\psi\|_{L_{|\breve{G}|^{1 / 2}}^{2}}^{2} \text {. }
$$

We have:

$$
\left\langle\check{\mathcal{L}}_{h} \check{\psi}, D_{\check{s}}^{2} \check{\psi}\right\rangle_{L_{|\check{G}|^{1 / 2}}^{2}}=\left\langle\check{\nabla}_{h} \check{g} \check{\nabla}_{h} \check{\psi}, D_{\check{s}}^{2} \check{\psi}\right\rangle_{L^{2}}
$$

We use the Green-Riemann formula to compute the quantity $\left\langle\check{\nabla}_{h} \check{g} \check{\nabla}_{h} \check{\psi}, D_{\check{s}}^{2} \check{\psi}\right\rangle_{L^{2}}$. Using the Sobolev injections and Corollary 4.5 , we control the boundary term by $O\left(h^{\infty}\right)\|\breve{\psi}\|_{L^{2}}\left\|D_{\breve{s}} \check{\psi}\right\|_{L^{2}}$ and we get:

$$
\left\langle\check{\nabla}_{h} \check{g} \check{\nabla}_{h} \check{\psi}, D_{\check{s}}^{2} \check{\psi}\right\rangle_{L^{2}}=\left\langle\check{g} \check{\nabla}_{h} \check{\psi}, \check{\nabla}_{h} D_{\check{s}}^{2} \check{\psi}\right\rangle_{L^{2}}+O\left(h^{\infty}\right)\left(\|\check{\psi}\|_{L^{2}}^{2}+\| D_{\check{s}} \check{\psi}_{L^{2}}^{2}\right)
$$

The following commutators computations are essentially algebraic:

$$
\begin{aligned}
& \left\langle\check{g} \check{\nabla}_{h} \check{\psi}, \check{\nabla}_{h} D_{\check{s}}^{2} \check{\psi}\right\rangle_{L^{2}}=\left\langle\check{g} \check{\nabla}_{h} \check{\psi},\left(D_{\check{s}} \check{\nabla}_{h}+\left[\check{\nabla}_{h}, D_{\check{s}}\right]\right) D_{\check{s}} \check{\psi}\right\rangle \\
& =\left\langle D_{\check{s}} \check{g} \check{\nabla}_{h} \check{\psi}, \check{\nabla}_{h} D_{\check{s}} \check{\psi}\right\rangle+\left\langle\check{g} \check{\nabla}_{h} \check{\psi},\left[\check{\nabla}_{h}, D_{\check{s}}\right] D_{\check{s}} \check{\psi}\right\rangle \\
& =\left\langle\check{g} \check{\nabla}_{h} D_{\check{s}} \check{\psi}, \check{\nabla}_{h} D_{\check{s}} \breve{\psi}\right\rangle+\left\langle\left(D_{\check{s}} \check{g}\right) \check{\nabla}_{h} \check{\psi}, \check{\nabla}_{h} D_{\check{s}} \check{\psi}\right\rangle \\
& -\left\langle\check{g}\left[\check{\nabla}_{h}, D_{\check{s}}\right] \check{\psi}, \check{\nabla}_{h} D_{\check{s}} \check{\psi}\right\rangle+\left\langle\check{g} \check{\nabla}_{h} \check{\psi},\left[\check{\nabla}_{h}, D_{\check{s}}\right] D_{\check{s}} \check{\psi}\right\rangle \\
& =\check{\mathcal{Q}}_{h}\left(D_{\check{s}} \check{\psi}\right)+\left\langle\left(D_{\check{s}} \check{g}\right) \check{\nabla}_{h} \check{\psi}, \check{\nabla}_{h} D_{\check{s}} \check{\psi}\right\rangle-\|\left[\check{\nabla}_{h}, D_{\check{s}}\right] \check{\psi}_{L_{\check{g}}^{2}}^{2} \\
& -\left\langle\check{g}\left[\check{\nabla}_{h}, D_{\check{s}}\right] \check{\psi}, D_{\check{s}} \check{\nabla}_{h} \check{\psi}\right\rangle+\left\langle\check{g} \check{\nabla}_{h} \check{\psi}, D_{\check{s}}\left[\check{\nabla}_{h}, D_{\check{s}}\right] \check{\psi}\right\rangle \\
& +\left\langle\check{g} \check{\nabla}_{h} \check{\psi},\left[\left[\check{\nabla}_{h}, D_{\check{s}}\right], D_{\check{s}}\right] \check{\psi}\right\rangle \text {. }
\end{aligned}
$$

We have:

$$
\begin{aligned}
& -\left\langle\check{g}\left[\check{\nabla}_{h}, D_{\breve{s}}\right] \check{\psi}, D_{\check{s}} \check{\nabla}_{h} \check{\psi}\right\rangle+\left\langle\check{g} \check{\nabla}_{h} \check{\psi}, D_{\check{s}}\left[\check{\nabla}_{h}, D_{\check{s}}\right] \breve{\psi}\right\rangle= \\
& \quad\left\langle\left[\check{\nabla}_{h}, D_{\check{s}}\right] \check{\psi},\left(D_{\check{s}} \check{g}\right) \check{\nabla}_{h} \check{\psi}\right\rangle-\left\langle D_{\check{s}}\left[\check{\nabla}_{h}, D_{\check{s}}\right] \check{\psi}, \check{g} \check{\nabla}_{h} \check{\psi}\right\rangle+\left\langle\check{g} \check{\nabla}_{h} \check{\psi}, D_{\check{s}}\left[\check{\nabla}_{h}, D_{\check{s}}\right] \check{\psi}\right\rangle .
\end{aligned}
$$


The two last terms are conjugate so that by taking the real part of $\left\langle\check{\mathcal{L}}_{h} \breve{\psi}, D_{\breve{s}}^{2} \breve{\psi}\right\rangle_{L_{|\breve{G}|^{1 / 2}}^{2}}$ we get (5.1).

We are led to estimate the different remainders due to the commutators.

Lemma 5.2. Let $N \geq 1$. There exist $h_{0}>0$ and $C>0$ such that, for $h \in\left(0, h_{0}\right)$ and $\check{\psi} \in \check{\mathfrak{E}}_{N}(h)$, we have:

$$
\begin{aligned}
& \left|\left\langle\check{g}\left[\check{\nabla}_{h}, D_{\check{s}}\right] \check{\psi},\left[\check{\nabla}_{h}, D_{\check{s}}\right] \check{\psi}\right\rangle\right| \leq C h^{2}\|\check{\psi}\|^{2}, \\
& \left|\left\langle\check{g} \check{\nabla}_{h} \check{\psi},\left[\left[\check{\nabla}_{h}, D_{\check{s}}\right], D_{\check{s}}\right] \breve{\psi}\right\rangle\right| \leq C h^{3 / 2}\|\check{\psi}\|^{2} \text {, } \\
& \left|\left\langle\left(D_{\check{s}} \check{g}\right) \check{\nabla}_{h} \check{\psi},\left[\check{\nabla}_{h}, D_{\check{s}}\right] \breve{\psi}\right\rangle\right| \leq C h^{2}\|\check{\psi}\|^{2} \text {, } \\
& \left|\left\langle\left(D_{\check{s}} \check{g}\right) \check{\nabla}_{h} \check{\psi}, \check{\nabla}_{h} D_{\check{s}} \check{\psi}\right\rangle\right| \leq C h\|\check{\psi}\| \sqrt{\check{\mathcal{Q}}_{h}\left(D_{\check{s}} \check{\psi}\right)}, \\
& h\left|\left\langle\left(D_{\check{s}}|\check{G}|^{1 / 2}\right) \check{\psi}, D_{\check{s}} \check{\psi}\right\rangle\right| \leq C h^{3 / 2}\left(\|\check{\psi}\|^{2}+\left\|D_{\check{s}} \check{\psi}\right\|^{2}\right) \text {. }
\end{aligned}
$$

Proof. We compute the first order commutator:

$$
\left[\check{\nabla}_{h}, D_{\check{s}}\right]=\left(\begin{array}{c}
-i h\left(\frac{\tau^{\prime}}{2 \tau}\right)^{\prime}\left(\check{z} D_{\check{z}}+D_{\check{z}} \check{z}\right)+\left(D_{\check{s}} \check{R}_{1}\right) \\
0 \\
-i h\left(\tau^{-1}\right)^{\prime} \tau_{0}
\end{array}\right) .
$$

We have $C_{1}>0$ such that $\left|D_{\check{s}} \check{R}_{1}\right| \leq C_{1}\left(\check{t}^{2}+\check{z}^{2}\right)$ (see Proposition 2.1). From Proposition 4.6 we get $\left\|\left[\check{\nabla}_{h}, D_{\breve{s}}\right] \breve{\psi}\right\| \leq C_{2} h$ with $C_{2}>0$ and the first upper bound follows. In the same way we get that $\left\|\left[\left[\check{\nabla}_{h}, D_{\check{s}}\right], D_{\check{s}}\right] \check{\psi}\right\| \leq C_{3} h$ with $C_{3}>0$ and since $\left\|\check{\nabla}_{h} \check{\psi}\right\| \leq \sqrt{\check{\mathcal{Q}}_{h}(\check{\psi})}=$ $O\left(h^{1 / 2}\right)\|\breve{\psi}\|$, we get the second upper bound. Using again Proposition 2.1, we deduce that $\left\|D_{\check{s} \check{g}}\right\| \leq C_{3}(|\check{t}|+|\check{z}|)$.

Lemma 5.3. There exist $h_{0}>0$ and $C>0$ such that, for $h \in\left(0, h_{0}\right)$ and $\check{\psi} \in \check{\mathfrak{E}}_{N}(h)$, we have:

$$
\left\|h D_{\check{t}} D_{\check{s}} \check{\psi}\right\|^{2}+\left\|h D_{\check{z}} D_{\check{s}} \check{\psi}\right\|^{2}+\left\|\left(h D_{\check{s}}-\check{t}\right) D_{\check{s}} \check{\psi}\right\|^{2} \leq C h\left\|D_{\check{s}} \check{\psi}\right\|^{2}+C h\|\check{\psi}\|^{2}
$$

Proof. We use Lemma 5.1 and Lemma 5.2 and we deduce:

$$
\check{\mathcal{Q}}_{h}\left(D_{\breve{s}} \check{\psi}\right) \leq \lambda\left\|D_{\check{s}} \check{\psi}\right\|^{2}+C h\|\check{\psi}\|^{2}+C h \check{\mathcal{Q}}_{h}\left(D_{\check{s}} \breve{\psi}\right)
$$

Therefore, we infer:

$$
(1-C h) \check{\mathcal{Q}}_{h}\left(D_{\check{s}} \check{\psi}\right) \leq\left(\nu\left(\alpha_{0}, \eta_{0}\right) h+C h^{3 / 2}\right)\left\|D_{\check{s}} \check{\psi}\right\|^{2}+C h\|\check{\psi}\|^{2}
$$

so that:

$$
\check{\mathcal{Q}}_{h}\left(D_{\check{s}} \check{\psi}\right) \leq\left(\nu\left(\alpha_{0}, \eta_{0}\right) h+\tilde{C} h^{3 / 2}\right)\left\|D_{\check{s}} \check{\psi}\right\|^{2}+\tilde{C} h\|\check{\psi}\|^{2} .
$$

Moreover, using (4.15) and Notation 4.4, we have:

$$
\check{\mathcal{Q}}_{h}\left(D_{\check{s}} \check{\psi}\right) \geq\left(1-C h^{1 / 2-\gamma}\right)\left\|\check{\nabla}_{h} \check{D}_{\check{s}} \check{\psi}\right\|^{2} .
$$


We write:

$$
\begin{aligned}
& \left\|\check{\nabla}_{h} \check{D}_{\check{s}} \check{\psi}\right\|^{2}=\left\|\left(h D_{\check{s}}-\check{t}+\eta_{0} h^{1 / 2}-\frac{h \tau^{\prime}}{2 \tau}\left(\check{z} D_{\check{z}}+D_{\check{z}} \check{z}\right)+\check{R}_{1}\right) D_{\check{s}} \breve{\psi}\right\|^{2}+\left\|h D_{\check{t}} D_{\check{s}} \check{\psi}\right\|^{2} \\
& +\left\|h \tau(\check{s})^{-1} \tau_{0} D_{\check{z}} D_{\check{s}} \breve{\psi}\right\|^{2} \\
& \geq \frac{1}{2} \check{\mathcal{Q}}_{h}^{\text {flat }}\left(D_{\check{s}} \psi\right)-2\left(C h^{2-4 \gamma}\left\|D_{\breve{s}} \check{\psi}\right\|^{2}+C h^{1-2 \gamma}\left\|h D_{\check{z}} D_{\check{s}} \check{\psi}\right\|^{2}+C h^{2}\|\check{\psi}\|^{2}\right)
\end{aligned}
$$

where $\check{\mathcal{Q}}_{h}^{\text {flat }}$ is defined in (4.21) and where we have used (4.15). We infer:

$$
\check{\mathcal{Q}}_{h}^{\text {flat }}\left(D_{\breve{s}} \psi\right) \leq \tilde{C} h\left\|D_{\breve{s}} \check{\psi}\right\|^{2}+\tilde{C} h\|\check{\psi}\|^{2}
$$

and the conclusion follows.

Proposition 5.4. There exist $h_{0}>0$ and $C>0$ such that, for $h \in\left(0, h_{0}\right)$ and $\check{\psi} \in \check{\mathfrak{E}}_{N}(h)$, we have:

$$
\left\|D_{\check{s}} \check{\psi}\right\| \leq C h^{-1 / 4}\|\check{\psi}\|
$$

Proof. Since we assume that Conjecture 1.9 is true, there exist $c_{0}>0, \varepsilon_{0}>0$ such that for $\left|\eta h^{1 / 2}\right| \leq \varepsilon_{0}$ :

$$
\nu\left(\alpha_{0}, \eta_{0}+\eta h^{1 / 2}\right) \geq \nu\left(\alpha_{0}, \eta_{0}\right)+h \frac{\partial_{\eta}^{2} \nu\left(\alpha_{0}, \eta_{0}\right)}{4} \eta^{2}
$$

and for $\left|\eta h^{1 / 2}\right| \geq \varepsilon_{0}$ :

$$
\nu\left(\alpha_{0}, \eta_{0}+h^{1 / 2} \eta\right) \geq \nu\left(\alpha_{0}, \eta_{0}\right)+c_{0}
$$

- Estimate near the minimum. We can notice that, with the estimates of Agmon, for all $\tilde{\psi} \in \check{\mathfrak{E}}_{N}(h):$

$$
\check{\mathcal{Q}}_{h}(\check{\psi}) \leq \lambda_{N}(h)\|\check{\psi}\|^{2}+O\left(h^{\infty}\right)\|\check{\psi}\|^{2}
$$

As in the proof of Proposition 4.7, we have:

$$
\check{\mathcal{Q}}(\check{\psi}) \geq\left\|h D_{\check{t}} \check{\psi}\right\|^{2}+\left\|h D_{\check{z}} \check{\psi}\right\|^{2}+\left\|\left(h D_{\check{s}}+\eta_{0} h^{1 / 2}-\check{t}\right) \check{\psi}\right\|^{2}-C h^{3 / 2}\|\check{\psi}\|^{2} .
$$

We use the Fourier transform $\mathcal{F}$ with respect to $\check{s}$ and the scaling associated to the variables $(\hat{t}, \hat{z})$ and we get:

$$
\int h \nu\left(\alpha_{0}, \eta_{0}+h^{1 / 2} \eta\right)|\mathcal{F} \check{\psi}|^{2} d \eta d \check{t} d \check{z} \leq\left(\nu\left(\alpha_{0}, \eta_{0}\right) h+C h^{3 / 2}\right)\|\mathcal{F} \check{\psi}\|^{2} .
$$

We split the integral of the 1.h.s. into two parts and we use (5.4) and (5.5) to infer:

$$
\begin{aligned}
& \int_{\left|\eta h^{1 / 2}\right| \geq \varepsilon_{0}}|\mathcal{F} \check{\psi}|^{2} d \eta d \check{t} d \check{z} \leq C h^{1 / 2}\|\check{\psi}\|^{2}, \\
& \int_{\left|\eta h^{1 / 2}\right| \leq \varepsilon_{0}} \eta^{2}|\mathcal{F} \check{\psi}|^{2} d \eta d \check{t} d \check{z} \leq C h^{-1 / 2}\|\check{\psi}\|^{2} .
\end{aligned}
$$


- Estimate away from the minimum. From (5.3) we get:

$$
\left\|\check{\nabla}_{h} \check{D}_{\check{s}} \check{\psi}\right\|^{2} \leq\left(\nu\left(\alpha_{0}, \eta_{0}\right) h+\tilde{C} h^{3 / 2-\gamma}\right)\left\|D_{\breve{s}} \check{\psi}\right\|^{2}+\tilde{C} h\|\check{\psi}\|^{2}
$$

We have:

$$
\begin{aligned}
& \left\|\check{\nabla}_{h} \check{D}_{\check{s}} \check{\psi}\right\|^{2}=\left\|\left(h D_{\check{s}}-\check{t}+\eta_{0} h^{1 / 2}-\frac{h \tau^{\prime}}{2 \tau}\left(\check{z} D_{\check{z}}+D_{\check{z}} \check{z}\right)+\check{R}_{1}\right) D_{\check{s}} \check{\psi}\right\|^{2}+\left\|\left(h D_{\check{t}}\right) D_{\check{s}} \check{\psi}\right\|^{2} \\
& +\left\|\left(h \tau(\check{s})^{-1} \tau_{0} D_{\check{z}}\right) D_{\check{s}} \check{\psi}\right\|^{2}
\end{aligned}
$$

We expand the squares and we shall estimate the double products. Let us just analyze the following term (we use Lemma 5.3 and (4.15)):

$$
\begin{aligned}
& \left|\Re\left\langle\left(h D_{\check{s}}-\check{t}+\eta_{0} h^{1 / 2}\right) D_{\check{s}} \check{\psi}, h \frac{\tau^{\prime}}{\tau}\left(\check{z} D_{\check{z}}+D_{\check{z}} \check{z}\right) D_{\check{s}} \check{\psi}+\check{R}_{1} D_{\check{s}} \check{\psi}\right\rangle\right| \\
& \leq\left\|\left(h D_{\check{s}}-\check{t}+\eta_{0} h^{1 / 2}\right) D_{\check{s}} \breve{\psi}\right\|\left(\left\|h \frac{\tau^{\prime}}{\tau}\left(\check{z} D_{\check{z}}+D_{\check{z}} \check{z}\right) D_{\check{s}} \check{\psi}\right\|+\left\|\check{R}_{1} D_{\check{s}} \check{\psi}\right\|\right) \\
& \leq C h^{1 / 2}\left(\left\|D_{\check{s}} \check{\psi}\right\|+\|\check{\psi}\|\right)\left(h^{1 / 2-\gamma}\left(h^{1 / 2}\left\|D_{\check{s}} \check{\psi}\right\|+h^{1 / 2}\|\check{\psi}\|\right)+h^{1-2 \gamma}\left\|D_{\check{s}} \check{\psi}\right\|\right) .
\end{aligned}
$$

We can deal with the other double products in the same way. We deduce:

$$
\left\|\left(h D_{\check{s}}-\check{t}+\eta_{0} h^{1 / 2}\right) D_{\check{s}} \check{\psi}\right\|^{2}+\left\|h D_{\check{t}} D_{\check{s}} \check{\psi}\right\|^{2}+\left\|h D_{\check{z}} D_{\check{s}} \check{\psi}\right\|^{2} \leq\left(\nu\left(\alpha_{0}, \eta_{0}\right) h+C h^{3 / 2-2 \gamma}\right)\left\|D_{\check{s}} \check{\psi}\right\|^{2}+C h\|\check{\psi}\|^{2} .
$$

We use the Fourier transform $\mathcal{F}$ to deduce:

$$
\int h \nu\left(\alpha_{0}, \eta_{0}+h^{1 / 2} \eta\right)|\eta \mathcal{F} \check{\psi}|^{2} d \eta d \check{t} d \check{z} \leq\left(\nu\left(\alpha_{0}, \eta_{0}\right) h+C h^{3 / 2-2 \gamma}\right)\|\eta \mathcal{F} \check{\psi}\|^{2}+C h\|\check{\psi}\|^{2} .
$$

We get:

and:

$$
\int_{\left|\eta h^{1 / 2}\right| \geq \varepsilon_{0}} c_{0} h|\eta \mathcal{F} \check{\psi}|^{2} d \eta d \check{t} d \check{z} \leq C h^{3 / 2-2 \gamma}\|\eta \mathcal{F} \check{\psi}\|^{2}+C h\|\check{\psi}\|^{2}
$$

$$
\int_{\left|\eta h^{1 / 2}\right| \geq \varepsilon_{0}}|\eta \mathcal{F} \check{\psi}|^{2} d \eta d \check{t} d \check{z} \leq \tilde{C} h^{1 / 2-2 \gamma}\|\eta \mathcal{F} \check{\psi}\|^{2}+\tilde{C}\|\check{\psi}\|^{2} .
$$

We combine this inequality with (5.8) and we get:

$$
\|\eta \mathcal{F} \breve{\psi}\|^{2} \leq \tilde{C} h^{1 / 2-2 \gamma}\|\eta \mathcal{F} \breve{\psi}\|^{2}+C h^{-1 / 2}\|\check{\psi}\|^{2}
$$

We deduce:

$$
\|\eta \mathcal{F} \check{\psi}\|^{2} \leq 2 C h^{-1 / 2}\|\check{\psi}\|^{2}
$$

5.2. Feshbach-Grushin projection. The result of Proposition 5.4 implies an approximation result for the eigenfunctions. Let us recall the scaling defined in (3.1):

$$
\check{s}=h^{1 / 4} \hat{s}, \quad \check{t}=h^{1 / 2} \hat{t}, \quad \check{z}=h^{1 / 2} \hat{z} .
$$

Notation 5.5. We will denote by $\widehat{\mathfrak{E}}_{N}(h)$ the set of the rescaled elements of $\check{\mathfrak{E}}_{N}(h)$. The elements of $\widehat{\mathfrak{E}}_{N}(h)$ will be denoted by $\hat{\psi}$. We recall that we denote by $\widehat{\mathcal{L}}_{h}$ the operator $h^{-1} \check{\mathcal{L}}_{h}$ in the rescaled coordinates. The corresponding quadratic form will be denoted by $\widehat{\mathcal{Q}}_{h}$. In these variables, Proposition 5.4 becomes:

$$
\left\|D_{\check{s}} \check{\psi}\right\| \leq C h^{-1 / 4}\|\check{\psi}\|
$$


Let us recall that $\gamma \in\left(0, \frac{1}{8}\right)$ is the scale of the cut-off function in (4.15). We have an approximation lemma:

Lemma 5.6. There exist $h_{0}>0$ and $C>0$ such that, for $h \in\left(0, h_{0}\right)$ and $\hat{\psi} \in \widehat{\mathfrak{E}}_{N}(h)$, we have:

$$
\begin{aligned}
& \left\|\hat{\psi}-\Pi_{0} \hat{\psi}\right\|+\left\|D_{\hat{t}}\left(\hat{\psi}-\Pi_{0} \hat{\psi}\right)\right\|+\left\|D_{\hat{z}}\left(\hat{\psi}-\Pi_{0} \hat{\psi}\right)\right\| \leq C h^{1 / 8}\|\hat{\psi}\| \\
& \left\|\hat{s}\left(\hat{\psi}-\Pi_{0} \hat{\psi}\right)\right\|+\left\|\hat{s} D_{\hat{t}}\left(\hat{\psi}-\Pi_{0} \hat{\psi}\right)\right\|+\left\|\hat{s} D_{\hat{z}}\left(\hat{\psi}-\Pi_{0} \hat{\psi}\right)\right\| \leq C h^{1 / 8-\gamma}(\|\hat{\psi}\|+(\|\hat{s} \hat{\psi}\|),
\end{aligned}
$$

where $\Pi_{0}$ is the projection on $u_{\eta_{0}}$ :

$$
\Pi_{0} \hat{\psi}=\left\langle\hat{\psi}, u_{\eta_{0}}\right\rangle_{\hat{t}, \hat{z}} u_{\eta_{0}}
$$

Proof. Let us prove the proposition for an $\hat{\psi}$ which comes from an eigenfunction associated with $\hat{\lambda}$. We have, using (5.6):

$$
\widehat{\mathcal{Q}}_{h}(\hat{\psi}) \leq\left(\nu\left(\alpha_{0}, \eta_{0}\right)+C h^{1 / 2}\right)\|\hat{\psi}\|^{2}+O\left(h^{\infty}\right)\|\hat{\psi}\|^{2} .
$$

In addition, as it was previously obtained in the proof of Proposition 4.7, we get:

$$
\widehat{\mathcal{Q}}_{h}(\hat{\psi}) \geq\left\|D_{\hat{t}} \hat{\psi}\right\|^{2}+\left\|D_{\hat{z}} \hat{\psi}\right\|^{2}+\left\|\left(h^{1 / 4} D_{\hat{s}}+\eta_{0}-\hat{t}\right) \hat{\psi}\right\|^{2}-C h^{1 / 2} .
$$

We apply Proposition 5.4 and we deduce:

$$
\left|\Re\left\langle h^{1 / 4}\left(\eta_{0}-\hat{t}\right) D_{\hat{s}} \hat{\psi}, \psi\right\rangle\right| \leq C h^{1 / 4}\|\hat{\psi}\|^{2} .
$$

Let us define $\widehat{\mathcal{L}}_{0}=\operatorname{Id}_{\hat{s}} \otimes\left(\mathcal{L}_{0}-\nu\left(\alpha_{0}, \eta_{0}\right)\right)$ and denote by $\widehat{Q}_{0}$ the associated quadratic form. We infer:

$$
0 \leq \hat{Q}_{0}(\hat{\psi})-\nu\left(\alpha_{0}, \eta_{0}\right)\|\hat{\psi}\|^{2} \leq C h^{1 / 4}\|\hat{\psi}\|^{2}
$$

and, since $\Pi_{0} \hat{\psi}$ is in the kernel of $\widehat{\mathcal{L}}_{0}$, we get:

$$
0 \leq \hat{Q}_{0}\left(\left(\mathrm{Id}-\Pi_{0}\right) \hat{\psi}\right)-\nu\left(\alpha_{0}, \eta_{0}\right)\left\|\left(\mathrm{Id}-\Pi_{0}\right) \hat{\psi}\right\|^{2} \leq C h^{1 / 4}\|\hat{\psi}\|^{2} .
$$

This is then standard, by using the simplicity of the lowest eigenvalue of $\mathcal{L}_{0}$, to deduce (5.10) (see [8, Corollary 4.10] and also [14]). To get (5.11), we write the "IMS" formula with weight $\hat{s}$ :

$$
\widehat{\mathcal{Q}}_{h}(\hat{s} \hat{\psi}) \leq \hat{\lambda}\|\hat{s} \hat{\psi}\|^{2}+C h^{1 / 2}\|\hat{\psi}\|^{2}+O\left(h^{\infty}\right)\|\hat{\psi}\|^{2} .
$$

Using the support of the functions (see (4.15)), we infer:

$$
\begin{aligned}
\widehat{\mathcal{Q}}_{h}(\hat{s} \hat{\psi}) \geq & \left(1-C h^{1 / 2-\gamma}\right)\left\|\left(h^{1 / 4} D_{\hat{s}}-\hat{t}+\eta_{0}-h^{1 / 2} \frac{\tau^{\prime}\left(h^{1 / 4} \hat{s}\right)}{2 \tau\left(h^{1 / 4} \hat{s}\right)}\left(\hat{z} D_{\hat{z}}+D_{\hat{z}} \hat{z}\right)+h^{-1 / 2} \hat{R}_{1}\right) \hat{s} \hat{\psi}\right\|^{2} \\
& +\left(1-C h^{1 / 2-\gamma}\right)\left\|D_{\hat{t}} \hat{s} \hat{\psi}\right\|^{2}+\left(1-C h^{1 / 2-\gamma}\right)\left\|\tau\left(h^{1 / 4} \hat{s}\right)^{-1} \tau_{0} D_{\hat{z}} \hat{s} \hat{\psi}\right\|^{2}
\end{aligned}
$$

As in the proof of Proposition 5.3, we deduce first:

$$
\left\|\left(h^{1 / 4} D_{\hat{s}}-\hat{t}+\eta_{0}\right) \hat{s} \hat{\psi}\right\|^{2}+\left\|D_{\hat{t}} \hat{s} \hat{\psi}\right\|^{2}+\left\|D_{\hat{z}} \hat{s} \hat{\psi}\right\|^{2} \leq C\|\hat{s} \hat{\psi}\|^{2}+C\|\hat{\psi}\|^{2}
$$

and then we analyze the remainders due to the double products in (5.13). Using (5.14), we get:

$$
\left|\left\langle\left(D_{\hat{s}}-\hat{t}+\eta_{0}\right) \hat{s} \hat{\psi}, h^{-1 / 2} \hat{R}_{1} \hat{s} \hat{\psi}\right\rangle\right| \leq C h^{-1 / 2} h^{1-2 \gamma}\|\hat{s} \hat{\psi}\|(\|\hat{s} \hat{\psi}\|+\|\hat{\psi}\|) .
$$


In addition, we get:

$$
\begin{aligned}
& \left|\left\langle\left(h^{1 / 4} D_{\hat{s}}-\hat{t}\right) \hat{s} \hat{\psi}, \frac{h^{1 / 2} \tau^{\prime}\left(h^{1 / 4} \hat{s}\right)}{2 \tau\left(h^{1 / 4} \hat{s}\right)}\left(\hat{z} D_{\hat{z}}+D_{\hat{z}} \hat{z}\right) \hat{s} \hat{\psi}\right\rangle\right| \\
& \leq C(\|\hat{s} \hat{\psi}\|+\|\hat{\psi}\|)\left(h^{1 / 2}\|\hat{s} \hat{\psi}\|+h^{1 / 2-\gamma}((\|\hat{s} \hat{\psi}\|+\|\hat{\psi}\|))\right)
\end{aligned}
$$

and:

$$
\begin{aligned}
& \left|\left\langle\frac{h^{1 / 2} \tau^{\prime}\left(h^{1 / 4} \hat{s}\right)}{2 \tau\left(h^{1 / 4} \hat{s}\right)}\left(\hat{z} D_{\hat{z}}+D_{\hat{z}} \hat{z}\right) \hat{s} \hat{\psi}, h^{-1 / 2} \hat{R}_{1}\right) \hat{s} \hat{\psi}\right\rangle \mid \\
& \leq C h^{-1 / 2} h^{1-2 \gamma}\|\hat{s} \hat{\psi}\|\left(h^{1 / 2}\|\hat{s} \hat{\psi}\|+h^{1 / 2-\gamma}(\|\hat{s} \hat{\psi}\|+\|\hat{\psi}\|)\right) .
\end{aligned}
$$

Therefore, from (5.13), we deduce:

$\widehat{\mathcal{Q}}_{h}(\hat{s} \hat{\psi}) \geq$

$$
\left(1-C h^{1 / 2-\gamma}\right)\left(\left\|\left(h^{1 / 4} D_{\hat{s}}-\hat{t}+\eta_{0}\right) \hat{s} \hat{\psi}\right\|^{2}+\left\|D_{\hat{t}} \hat{s} \hat{\psi}\right\|^{2}+\left\|D_{\hat{z}} \hat{s} \hat{\psi}\right\|^{2}-C h^{1 / 2-2 \gamma}\left(\|\hat{s} \hat{\psi}\|^{2}+\|\hat{\psi}\|^{2}\right)\right) .
$$

so that:

$\left\|\left(h^{1 / 4} D_{\hat{s}}-\hat{t}+\eta_{0}\right) \hat{s} \hat{\psi}\right\|^{2}+\left\|D_{\hat{t}} \hat{s} \hat{\psi}\right\|^{2}+\left\|D_{\hat{z}} \hat{s} \hat{\psi}\right\|^{2} \leq \nu\left(\alpha_{0}, \eta_{0}\right)\|\hat{s} \hat{\psi}\|^{2}+C h^{1 / 2-\gamma}\left(\|\hat{s} \hat{\psi}\|^{2}+\|\hat{\psi}\|^{2}\right)$.

We have finally to analyze the term:

$$
\left\langle h^{1 / 4} D_{\hat{s}} \hat{s} \hat{\psi},\left(-\hat{t}+\eta_{0}\right) \hat{s} \hat{\psi}\right\rangle .
$$

We get:

$$
\left|\left\langle h^{1 / 4} D_{\hat{s}} \hat{s} \hat{\psi},\left(-\hat{t}+\eta_{0}\right) \hat{s} \hat{\psi}\right\rangle\right| \leq C\left(h^{1 / 4}\left(\|\hat{\psi}\|^{2}+\|\hat{s} \hat{\psi}\|^{2}\right)+h^{1 / 4-2 \gamma}\left(\|\hat{\psi}\|^{2}+\|\hat{s} \hat{\psi}\|^{2}\right)\right) .
$$

Therefore, we have proved the following estimate:

$$
\left\|\left(\hat{t}-\eta_{0}\right) \hat{s} \hat{\psi}\right\|^{2}+\left\|D_{\hat{t}} \hat{s} \hat{\psi}\right\|^{2}+\left\|D_{\hat{z}} \hat{s} \hat{\psi}\right\|^{2} \leq \nu\left(\alpha_{0}, \eta_{0}\right)\|\hat{s} \hat{\psi}\|^{2}+C h^{1 / 4-2 \gamma}\left(\|\hat{s} \hat{\psi}\|^{2}+\|\hat{\psi}\|^{2}\right) .
$$

We recognize $\widehat{Q}_{0}(\hat{s} \hat{\psi})$ in the 1.h.s. and the conclusion is standard.

5.3. Control of $\check{s}$. The aim of this section is to obtain a control with respect to $\hat{s}$. This estimate can not be obtained directly since the dependence on $\hat{s}$ comes as $\hat{s}^{2} D_{\hat{z}}^{2}$ in the operator. Therefore in order to catch the variable $\hat{s}$, we will use the projection to replace (modulo correction terms) $D_{\hat{z}}^{2}$ by a positive number when acting on the eigenfunctions.

Proposition 5.7. There exist $h_{0}>0$ and $C>0$ such that, for $h \in\left(0, h_{0}\right)$ and $\check{\psi} \in \check{\mathfrak{E}}_{N}(h)$, we have:

$$
\|\check{s} \breve{\psi}\| \leq C h^{1 / 4}\|\check{\psi}\|
$$

Proof. It is equivalent to prove that:

$$
\|\hat{s} \hat{\psi}\| \leq C\|\hat{\psi}\|
$$

The proof of Proposition 4.7 provides the inequality:

$$
\left\|D_{\hat{t}} \hat{\psi}\right\|^{2}+\left\|\tau_{0} \tau\left(h^{1 / 4} \hat{s}\right)^{-1} D_{\hat{z}} \hat{\psi}\right\|^{2}+\left\|\left(h^{1 / 4} D_{\hat{s}}+\eta_{0}-\hat{t}\right) \hat{\psi}\right\|^{2} \leq\left(\nu\left(\alpha_{0}, \eta_{0}\right)+C h^{1 / 2}\right)\|\hat{\psi}\|^{2} .
$$

From the non-degeneracy of the maximum of $\alpha$, we deduce the existence of $c>0$ such that:

$$
\left\|\tau_{0} \tau\left(h^{1 / 4} \hat{s}\right)^{-1} D_{\hat{z}} \hat{\psi}\right\|^{2} \geq\left\|D_{\hat{z}} \hat{\psi}\right\|^{2}+c h^{1 / 2}\left\|\hat{s} D_{\hat{z}} \hat{\psi}\right\|^{2}
$$


so that we have:

$$
c h^{1 / 2}\left\|\hat{s} D_{\hat{z}} \hat{\psi}\right\|^{2} \leq C h^{1 / 2}\|\hat{\psi}\|^{2}
$$

and:

$$
\left\|\hat{s} D_{\hat{z}} \hat{\psi}\right\| \leq \tilde{C}\|\hat{\psi}\|
$$

It remains to use Lemma 5.6 and especially (5.11). In particular, we have:

$$
\left\|\hat{s} D_{\hat{z}}\left(\hat{\psi}-\Pi_{0} \hat{\psi}\right)\right\| \leq C h^{1 / 8-\gamma}(\|\hat{\psi}\|+\|\hat{s} \hat{\psi}\|) .
$$

We infer:

Let us write

$$
\left\|\hat{s} D_{\hat{z}} \Pi_{0} \hat{\psi}\right\| \leq \tilde{C}\|\hat{\psi}\|+C h^{1 / 8-\gamma}(\|\hat{\psi}\|+\|\hat{s} \hat{\psi}\|) .
$$

$$
\Pi_{0} \hat{\psi}=f_{h}(\hat{s}) u_{\eta_{0}}(\hat{t}, \hat{z})
$$

We have:

$$
\left\|\hat{s} D_{\hat{z}} \Pi_{0} \hat{\psi}\right\|=\left\|D_{\hat{z}} u_{\eta_{0}}\right\|\left\|\hat{s} f_{h}\right\|_{L^{2}(d \hat{s})}=\left\|D_{\hat{z}} u_{\eta_{0}}\right\|\left\|\hat{s} f_{h} u_{\eta_{0}}\right\|=\left\|D_{\hat{z}} u_{\eta_{0}}\right\|\left\|\hat{s} \Pi_{0} \hat{\psi}\right\| .
$$

We use again Lemma 5.6 to get:

$$
\left\|\hat{s} D_{\hat{z}} \Pi_{0} \hat{\psi}\right\|=\left\|D_{\hat{z}} u_{\eta_{0}}\right\|\|\hat{s} \hat{\psi}\|+O\left(h^{1 / 8-\gamma}\right)(\|\hat{\psi}\|+\|\hat{s} \hat{\psi}\|) .
$$

We deduce:

$$
\left\|D_{\hat{z}} u_{\eta_{0}}\right\|\|\hat{s} \hat{\psi}\| \leq \tilde{C}\|\hat{\psi}\|+2 C h^{1 / 8-\gamma}(\|\hat{\psi}\|+(\|\hat{s} \hat{\psi}\|)
$$

and the conclusion follows.

\section{REDUCTION TO THE BORN-OPPENHEIMER APPROXIMATION}

This section is devoted to the proof of the following proposition.

Proposition 6.1. We assume that Conjecture 1.9 is true. We also assume Assumptions 1.12 and 1.14. For all $n \geq 1$, there exists $h_{0}>0$ such that, for $h \in\left(0, h_{0}\right)$ :

$$
\lambda_{n}(h) \geq \mu_{0, n} h+\mu_{2, n} h^{3 / 2}+o\left(h^{3 / 2}\right),
$$

where $\mu_{0, n}$ et $\mu_{2, n}$ are given in Theorem 1.16.

In particular, we show that we can reduce our spectral investigation to the study of a BornOppenheimer approximation.

Proposition 6.2. There exists $h_{0}>0$ such that for $h \in\left(0, h_{0}\right)$ and $\hat{\psi} \in \widehat{\mathfrak{E}}_{N}(h)$, we have:

$\widehat{\mathcal{Q}}_{h}(\hat{\psi}) \geq\left\|D_{\hat{t}} \hat{\psi}\right\|^{2}+\left\|D_{\hat{z}} \hat{\psi}\right\|^{2}+\left\|\left(h^{1 / 4} D_{\hat{s}}-\hat{t}+\eta_{0}\right) \hat{\psi}\right\|^{2}+h^{1 / 2} \tau_{0}^{-1} \kappa\left\|D_{\hat{z}} u_{\eta_{0}}\right\|^{2} \hat{s}^{2}+\tilde{\omega}_{0} h^{1 / 2}\|\hat{\psi}\|^{2}$ $+o\left(h^{1 / 2}\right)\|\hat{\psi}\|^{2}$

with:

$$
\tilde{\omega}_{0}=\left\langle\left(2\left(\eta_{0}-\hat{t}\right) \hat{r}_{1} u_{\eta_{0}}, u_{\eta_{0}}\right\rangle_{L^{2}(d \hat{t} d \hat{z})}+\int_{\mathcal{S}_{\alpha}} \frac{\hat{l}}{2} \hat{P} u_{\eta_{0}} \hat{P} u_{\eta_{0}} d \hat{t} d \hat{z}+\int_{\mathcal{S}_{\alpha}} \hat{L} \hat{P} u_{\eta_{0}} \hat{P} u_{\eta_{0}} d \hat{t} d \hat{z}\right.
$$

where $\hat{P}, \hat{l}, \hat{L}$ and $\hat{r}_{1}$ are homogeneous polynomials defined in (3.5) and (??) (see also Section 2). 
Proof. We can write:

$$
\check{\mathcal{Q}}_{h}(\check{\psi})=\int\left(\check{g} \check{\nabla}_{h} \check{\psi}\right) \cdot\left(\check{\nabla}_{h} \check{\psi}\right) d \check{s} d \check{t} d \check{z}
$$

By Proposition 2.1, we have:

$$
\check{g}=\mathrm{ld}+\frac{\check{l}}{2} \mathrm{ld}+\check{L}+(|\check{t}|+|\check{z}|) \mathcal{O}_{1}
$$

where the notation $\mathcal{O}_{1}$ is defined in Proposition 2.1. Let us estimate the following remainder:

$$
\mathcal{R}=\int\left\{|\check{s}|(|\check{t}|+|\check{z}|)+|\check{t}|^{2}+|\check{z}|^{2}\right\}\left\|\check{\nabla}{ }_{h} \check{\psi}\right\|^{2} d \check{s} d \check{t} d \check{z} .
$$

Using the support of the functions, we have:

$$
|\mathcal{R}| \leq \int\left\{C h^{1 / 8-\gamma}(|\check{t}|+|\check{z}|)+|\check{t}|^{2}+|\check{z}|^{2}\right\}\left\|\check{\nabla}_{h} \check{\psi}\right\|^{2} d \check{s} d \check{t} d \check{z} .
$$

Then, we recall that:

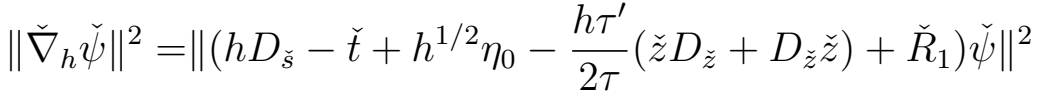

$$
\begin{aligned}
& +\left\|h D_{\hat{t}} \check{\psi}\right\|^{2}+\left\|h \tau_{0} \tau^{-1}(\check{s}) D_{\check{z}} \check{\psi}\right\|^{2} .
\end{aligned}
$$

It easily follows that:

$$
\begin{aligned}
\left\|\check{\nabla}_{h} \check{\psi}\right\|^{2} \leq & C\left(\left\|\left(h D_{\check{s}}-\check{t}+h^{1 / 2} \eta_{0}\right) \check{\psi}\right\|^{2}+\left\|h D_{\hat{t}} \check{\psi}\right\|^{2}+\left\|h D_{\check{z}} \check{\psi}\right\|^{2}\right) \\
& +C h^{2}\left\|\left(\check{z} D_{\check{z}}+D_{\check{z}} \check{z}\right) \check{\psi}\right\|^{2}+C\left\|\check{R}_{1} \check{\psi}\right\|^{2} .
\end{aligned}
$$

The estimates of Agmon with respect to $\check{t}$ and $\check{z}$ (see Proposition 4.6) jointly with the Taylor expansion of $\check{R}_{1}$ given in (2.5) imply that:

$$
\left\|\check{R}_{1} \check{\psi}\right\|^{2} \leq C h^{2}\|\check{\psi}\|^{2}
$$

We can deal with the other terms in the same way to get:

$$
\left|\mathcal{R}_{1}\right| \leq\left(C h^{1 / 8-\gamma} h^{3 / 2}+C h^{2}\right)\|\check{\psi}\|^{2} .
$$

We infer from (6.2) and (6.3) that:

$$
\check{\mathcal{Q}}_{h}(\check{\psi}) \geq \int\left(\mathrm{Id}+\frac{\check{l}}{2} \mathrm{Id}+\check{L}\right) \check{\nabla}_{h} \check{\psi} \cdot \check{\nabla}_{h} \check{\psi} d \check{s} d \check{t} d \check{z}+o\left(h^{3 / 2}\right)\|\check{\psi}\|^{2}
$$

It follows that:

$$
\check{\mathcal{Q}}_{h}(\check{\psi}) \geq\left\|\check{\nabla}_{h} \check{\psi}\right\|^{2}+\int\left(\frac{\check{l}}{2} \mathrm{ld}+\check{L}\right) \check{\nabla}_{h} \check{\psi} \cdot \check{\nabla}_{h} \check{\psi} d \check{s} d \check{t} d \check{z}+o\left(h^{3 / 2}\right)\|\check{\psi}\|^{2} .
$$


- Terms involving the metrics. With the same argument as previously, we can write:

$$
\begin{aligned}
& \int\left(\frac{l}{2} \mathrm{ld}+\check{L}\right) \check{\nabla}_{h} \check{\psi} \cdot \check{\nabla}_{h} \check{\psi} d \check{s} d \check{t} d \check{z} \\
& \geq \int\left(\frac{\grave{l}}{2} \mathrm{ld}+\check{L}\right)\left(\begin{array}{c}
h D_{\check{s}}-\check{t}+h^{1 / 2} \eta_{0} \\
h D_{\check{t}} \\
h \tau_{0} \tau^{-1} D_{\check{z}}
\end{array}\right) \check{\psi} \cdot\left(\begin{array}{c}
h D_{\check{s}}-\check{t}+h^{1 / 2} \eta_{0} \\
h D_{\check{t}} \\
h \tau_{0} \tau^{-1} D_{\check{z}}
\end{array}\right) \check{\psi} d \check{s} d \check{t} d \check{z} \\
& +o\left(h^{3 / 2}\right)\|\check{\psi}\|^{2} .
\end{aligned}
$$

Let us analyze the different terms involving $D_{\check{z}}$. Since we have $\tau_{0} \tau^{-1}(\check{s})=1+O\left(\check{s}^{2}\right)$, we have to control a term in the form:

$$
\int|\check{s}|^{2}(|\check{t}|+|\check{z}|)\left|h D_{\check{z}} \check{\psi}\right|\left(\left|\left(h D_{\check{s}}-\check{t}+h^{1 / 2} \eta_{0}\right) \check{\psi}\right|+\left|h D_{\check{t}} \check{\psi}\right|+\left|h D_{\check{z}} \check{\psi}\right|\right) d \check{s} d \check{t} d \check{z} .
$$

This term is controlled by $C\left(h^{1 / 8-\gamma}\right)^{2} h^{1 / 2} h\|\check{\psi}\|^{2}$. This allows to write:

$$
\begin{aligned}
& \int\left(\frac{l}{2} \mathrm{Id}+\check{L}\right) \check{\nabla}_{h} \check{\psi} \cdot \check{\nabla}_{h} \check{\psi} d \check{s} d \check{t} d \check{z} \\
& \geq \int\left(\frac{\grave{l}}{2} \mathrm{Id}+\check{L}\right)\left(\begin{array}{c}
h D_{\check{s}}-\check{t}+h^{1 / 2} \eta_{0} \\
h D_{\check{t}} \\
h D_{\check{z}}
\end{array}\right) \check{\psi} \cdot\left(\begin{array}{c}
h D_{\check{s}}-\check{t}+h^{1 / 2} \eta_{0} \\
h D_{\check{t}} \\
h D_{\check{z}}
\end{array}\right) \check{\psi} d \check{s} d \check{t} d \check{z} \\
& +o\left(h^{3 / 2}\right)\|\check{\psi}\|^{2} .
\end{aligned}
$$

Let us now deal with the terms involving $D_{\breve{s}}$. Such terms appear in integrals in the form:

$$
\begin{gathered}
\int \check{m}_{1}(\check{t}, \check{z})\left(h D_{\check{s}}-\check{t}+\eta_{0} h^{1 / 2}\right) \check{\psi} \overline{\left(h D_{\check{s}}-\check{t}+\eta_{0} h^{1 / 2}\right) \check{\psi}} d \check{s} d \check{t} d \check{z}, \\
\int \check{m}_{2}(\check{t}, \check{z})\left(h D_{\check{s}}-\check{t}+\eta_{0} h^{1 / 2}\right) \check{\psi} \overline{h D_{\check{t}} \check{\psi}} d \check{s} d \check{t} d \check{z}
\end{gathered}
$$

or:

$$
\int \check{m}_{3}(\check{t}, \check{z})\left(h D_{\check{s}}-\check{t}+\eta_{0} h^{1 / 2}\right) \check{\psi} \overline{h D_{\check{z}} \check{\psi}} d \check{s} d \check{t} d \check{z},
$$

where the $\check{m}_{j}$ are linear. Let us just analyze the first one, the other being similar. We have to estimate a double product:

$$
\left|\int \check{m}_{1}(\check{t}, \check{z}) h D_{\check{s}} \check{\psi} \overline{\left(-\check{t}+\eta_{0} h^{1 / 2}\right) \check{\psi}} d \check{s} d \check{t} d \check{z}\right| \leq C\left\|h D_{\check{s}} \check{\psi}\right\| h\|\check{\psi}\|,
$$

where we have used the normal estimates of Agmon. It remains to use Proposition 5.4 and we infer that:

$$
\begin{aligned}
& \int\left(\frac{\check{l}}{2} \mathrm{Id}+\check{L}\right) \check{\nabla}_{h} \check{\psi} \cdot \check{\nabla}_{h} \check{\psi} d \check{s} d \check{t} d \check{z} \\
& \geq \int\left(\frac{\grave{l}}{2} \mathrm{Id}+\check{L}\right)\left(\begin{array}{c}
-\check{t}+h^{1 / 2} \eta_{0} \\
h D_{\check{t}} \\
h D_{\check{z}}
\end{array}\right) \check{\psi} \cdot\left(\begin{array}{c}
-\check{t}+h^{1 / 2} \eta_{0} \\
h D_{\check{t}} \\
h D_{\check{z}}
\end{array}\right) \check{\psi} d \check{s} d \check{t} d \check{z} \\
& +o\left(h^{3 / 2}\right)\|\check{\psi}\|^{2} .
\end{aligned}
$$


- Terms involving $\check{R}_{1}$. Let us deal with the first term in (6.7). We recall (6.5). We notice that:

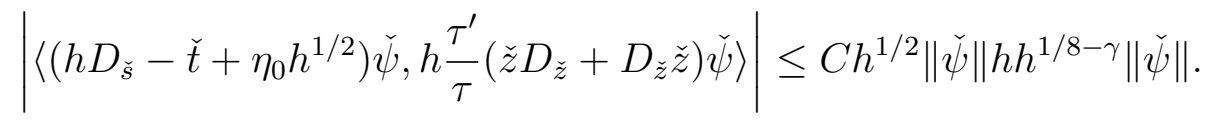

In the same way, we find:

$$
\left|\left\langle\check{R}_{1} \check{\psi}, h \frac{\tau^{\prime}}{\tau}\left(\check{z} D_{\check{z}}+D_{\check{z}} \check{z}\right) \check{\psi}\right\rangle\right|=o\left(h^{3 / 2}\right)\|\check{\psi}\|^{2} .
$$

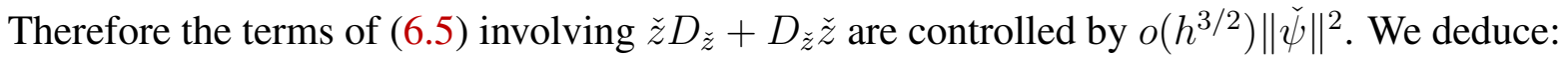

$$
\begin{aligned}
& \left\|\check{\nabla}_{h} \check{\psi}\right\|^{2} \geq\left\|\left(h D_{\check{s}}-\check{t}+\eta_{0} h^{1 / 2}+\check{R}_{1}\right) \check{\psi}\right\|^{2}+\left\|h D_{\check{t}} \check{\psi}\right\|^{2}+\left\|\tau_{0} \tau^{-1} h D_{\check{z}} \check{\psi}\right\|^{2} \\
& +o\left(h^{3 / 2}\right)\|\check{\psi}\|^{2} \text {. }
\end{aligned}
$$

Thanks to Proposition 5.4 and the estimate (6.6), we infer:

$$
\left|\left\langle h D_{\check{s}} \check{\psi}, \check{R}_{1} \check{\psi}\right\rangle\right| \leq C h^{3 / 4} h\|\check{\psi}\|^{2} \text {. }
$$

We deduce the inequality:

$$
\begin{aligned}
& \left\|\check{\nabla}_{h} \check{\psi}\right\|^{2} \geq\left\|\left(h D_{\breve{s}}-\check{t}+\eta_{0} h^{1 / 2}\right) \check{\psi}\right\|^{2}+\left\|h D_{\check{t}} \check{\psi}\right\|^{2}+\left\|\tau_{0} \tau^{-1} h D_{\check{z}} \check{\psi}\right\|^{2} \\
& +2\left\langle\left(-\check{t}+\eta_{0} h^{1 / 2}\right) \check{\psi}, \check{R}_{1} \check{\psi}\right\rangle+o\left(h^{3 / 2}\right)\|\check{\psi}\|^{2} .
\end{aligned}
$$

Let us now notice that:

$$
\tau_{0} \tau^{-1}(\check{s}) \geq 1+\tau_{0}^{-1} \kappa \check{s}^{2}-C|\check{s}|^{3}
$$

We deduce that:

$$
\left\|\tau_{0} \tau^{-1} h D_{\check{z}} \check{\psi}\right\|^{2} \geq\left\|h D_{\check{z}} \check{\psi}\right\|^{2}+\tau_{0}^{-1} \kappa\left\|\check{s} h D_{\check{z}} \check{\psi}\right\|^{2}-C h^{1 / 8-\gamma}\left\|\check{s} h D_{\check{z}} \check{\psi}\right\|^{2} .
$$

- Rescaling. We now gather (6.7), (6.8), (6.10) and (6.11) and we use the rescaled coordinates $(\hat{s}, \hat{t}, \hat{z})$ (defined in (3.5)) to get:

$$
\begin{aligned}
\widehat{\mathcal{Q}}_{h}(\hat{\psi}) \geq & \left\|\left(h^{1 / 4} D_{\hat{s}}-\hat{t}+\eta_{0}\right) \hat{\psi}\right\|^{2}+\left\|D_{\hat{t}} \hat{\psi}\right\|^{2}+\left\|D_{\hat{z}} \hat{\psi}\right\|^{2}+h^{1 / 2} \tau_{0}^{-1} \kappa\left\|\hat{s} D_{\hat{z}} \hat{\psi}\right\|^{2} \\
& +2 h^{1 / 2}\left\langle\left(-\hat{t}+\eta_{0}\right) \hat{\psi}, \hat{r}_{1} \check{\psi}\right\rangle-C h^{1 / 2} h^{1 / 8-\gamma}\left\|\hat{s} D_{\hat{z}} \hat{\psi}\right\|^{2} \\
& +\int\left(\frac{\check{l}}{2} \mathrm{Id}+\check{L}\right) \hat{P} \hat{\psi} \cdot \hat{P} \hat{\psi} d \check{s} d \check{t} d \check{z}+o\left(h^{1 / 2}\right)\|\hat{\psi}\|^{2} .
\end{aligned}
$$

The main idea is now to replace $\hat{\psi}$ by $\Pi_{0} \hat{\psi}$ in the terms of order $h^{1 / 2}$ in order to make the terms coming from the metrics disappear and get the constant $\tilde{\omega}_{0}$. This procedure aims at averaging the lower order terms of the symbol of the operator with respect to the eigenfunction of the leading operator. We recall Proposition 5.4 and Lemma 5.6. This implies that:

$$
\left|\left\|\hat{s} D_{\hat{z}} \hat{\psi}\right\|-\left\|D_{\hat{z}} u_{\eta_{0}}\right\|\|\hat{s} \hat{\psi}\|\right| \leq C h^{1 / 8-\gamma}\|\hat{\psi}\| .
$$

On the one hand we deduce:

$$
\left\|\hat{s} D_{\hat{z}} \hat{\psi}\right\| \leq C\|\hat{\psi}\|
$$

and on the other hand:

$$
\left\|\hat{s} D_{\hat{z}} \hat{\psi}\right\|^{2} \geq\left\|D_{\hat{z}} u_{\eta_{0}}\right\|^{2}\|\hat{s} \hat{\psi}\|^{2}-C h^{1 / 8-\gamma}\|\hat{\psi}\|^{2}
$$


We infer:

$$
\begin{aligned}
\widehat{\mathcal{Q}}_{h}(\hat{\psi}) \geq & \left\|\left(h^{1 / 4} D_{\hat{s}}-\hat{t}+\eta_{0}\right) \hat{\psi}\right\|^{2}+\left\|D_{\hat{t}} \hat{\psi}\right\|^{2}+\left\|D_{\hat{z}} \hat{\psi}\right\|^{2}+h^{1 / 2} \tau_{0}^{-1} \kappa\left\|D_{\hat{z}} u_{\eta_{0}}\right\|^{2}\|\hat{s} \hat{\psi}\|^{2} \\
& +2 h^{1 / 2} \Re\left(\left\langle\left(-\hat{t}+\eta_{0}\right) \hat{\psi}, \hat{r}_{1} \hat{\psi}\right\rangle\right) \\
& +\int\left(\frac{\check{l}}{2} \mathrm{Id}+\check{L}\right) \hat{P} \hat{\psi} \cdot \hat{P} \hat{\psi} d \hat{s} d \hat{t} d \hat{z}+o\left(h^{1 / 2}\right)\|\hat{\psi}\|^{2} .
\end{aligned}
$$

We finally deal only with the term involving $\hat{r}_{1}$ defined in (3.7), the other being similar. We use Lemma 5.6 and especially (5.10). We can write:

$$
\left\langle\left(-\hat{t}+\eta_{0}\right) \hat{\psi}, \hat{r}_{1} \hat{\psi}\right\rangle \text {. }
$$

By the normal estimates of Agmon, we have:

$$
\left\|\hat{r}_{1}\left(-\hat{t}+\eta_{0}\right) \hat{\psi}\right\| \leq C\|\hat{\psi}\|
$$

and with (5.11) we get:

$$
\left\langle\hat{r}_{1}\left(-\hat{t}+\eta_{0}\right) \hat{\psi}, \hat{\psi}\right\rangle=\left\langle\hat{r}_{1}\left(-\hat{t}+\eta_{0}\right) \hat{\psi}, \Pi_{0} \hat{\psi}\right\rangle+o(1)\|\hat{\psi}\|^{2} .
$$

Using the decay properties of $u_{\eta_{0}}$ and (5.10), we have:

$$
\left\|\hat{r}_{1}\left(-\hat{t}+\eta_{0}\right) \Pi_{0} \hat{\psi}\right\| \leq C\|\hat{\psi}\| .
$$

It follows that:

$$
\left\langle\left(-\hat{t}+\eta_{0}\right) \hat{\psi}, \hat{r}_{1} \hat{\psi}\right\rangle=\left\langle\left(-\hat{t}+\eta_{0}\right) \Pi_{0} \hat{\psi}, \hat{r}_{1} \Pi_{0} \hat{\psi}\right\rangle+o(1)\|\hat{\psi}\|^{2} .
$$

Jointly with (6.12) and the definition of $\tilde{\omega}_{0}$, this provides the conclusion.

- Born-Oppenheimer approximation and conclusion. In this last paragraph we prove Proposition 6.1. Let us now consider the inequality of Proposition 6.2. There exists $h_{0}>0$ such that for $h \in\left(0, h_{0}\right)$ and $\hat{\psi} \in \widehat{\mathfrak{E}}_{N}(h)$, we have:

$$
\begin{aligned}
& \left\|D_{\hat{t}} \hat{\psi}\right\|^{2}+\left\|D_{\hat{z}} \hat{\psi}\right\|^{2}+\left\|\left(h^{1 / 4} D_{\hat{s}}-\hat{t}+\eta_{0}\right) \hat{\psi}\right\|^{2}+h^{1 / 2} \tau_{0}^{-1} \kappa\left\|D_{\hat{z}} u_{\eta_{0}}\right\|^{2} \hat{s}^{2}+\tilde{\omega}_{0} h^{1 / 2}\|\hat{\psi}\|^{2} \\
& \leq \hat{\lambda}_{N}(h) \int\left(1+h^{1 / 2} \frac{\hat{l}}{2}\right)|\hat{\psi}|^{2} d \hat{s} d \hat{t} d \hat{z}+o\left(h^{1 / 2}\right)\|\hat{\psi}\|^{2} .
\end{aligned}
$$

Using again (5.10) and $\hat{\lambda}_{N}(h)=\nu\left(\alpha_{0}, \eta_{0}\right)+o\left(h^{1 / 2}\right)$, we infer (see (3.13) and (6.1)), for all $\hat{\psi} \in \widehat{\mathfrak{E}}_{N}(h):$

$$
\begin{aligned}
& \left\|D_{\hat{t}} \hat{\psi}\right\|^{2}+\left\|D_{\hat{z}} \hat{\psi}\right\|^{2}+\left\|\left(h^{1 / 4} D_{\hat{s}}-\hat{t}+\eta_{0}\right) \hat{\psi}\right\|^{2}+h^{1 / 2} \tau_{0}^{-1} \kappa\left\|D_{\hat{z}} u_{\eta_{0}}\right\|^{2} \hat{s}^{2}+\omega_{0} h^{1 / 2}\|\hat{\psi}\|^{2} \\
& \leq \hat{\lambda}_{N}(h) \int|\hat{\psi}|^{2} d \hat{s} d \hat{t} d \hat{z}+o\left(h^{1 / 2}\right)\|\hat{\psi}\|^{2} .
\end{aligned}
$$

Let us consider the operator which appears in the left hand side:

$$
D_{\hat{t}}^{2}+D_{\hat{z}}^{2}+\left(h^{1 / 4} D_{\hat{s}}-\hat{t}+\eta_{0}\right)^{2}+h^{1 / 2} \tau_{0}^{-1} \kappa\left\|D_{\hat{z}} u_{\eta_{0}}\right\|^{2} \hat{s}^{2}+\omega_{0} h^{1 / 2} .
$$

After Fourier transform with respect to $\hat{s}$, the operator (6.15) becomes:

$$
D_{\hat{t}}^{2}+D_{\hat{z}}^{2}+\left(h^{1 / 4} \xi-\hat{t}+\eta_{0}\right)^{2}+h^{1 / 2} \tau_{0}^{-1} \kappa\left\|D_{\hat{z}} u_{\eta_{0}}\right\|^{2} D_{\xi}^{2}+\omega_{0} h^{1 / 2} .
$$


To see why the Born-Oppeheimer formalism applies to the operator (6.16), let us perform the scaling $\tilde{\xi}=h^{1 / 4} \xi$. We introduce:

$$
\mathcal{L}_{h}^{\mathrm{BO}}=D_{\hat{t}}^{2}+D_{\hat{z}}^{2}+\left(\tilde{\xi}-\hat{t}+\eta_{0}\right)^{2}+h \tau_{0}^{-1} \kappa\left\|D_{\hat{z}} u_{\eta_{0}}\right\|^{2} D_{\tilde{\xi}}^{2}+\omega_{0} h^{1 / 2} .
$$

The operator $\mathcal{L}_{h}^{\mathrm{BO}}$ is semiclassical with respect to the variable $\tilde{\xi}$. The Born-Oppenheimer theory applies (see [30] and [10]) and the $n$-th eigenvalue $\lambda_{n}^{\mathrm{BO}}(h)$ of $\mathcal{L}_{h}^{\mathrm{BO}}$ satisfies:

$$
\lambda_{n}^{\mathrm{BO}}(h)=\nu\left(\alpha_{0}, \eta_{0}\right)+\mu_{2, n} h^{1 / 2}+o\left(h^{1 / 2}\right) .
$$

It remains to apply the min-max principle to the $N$-dimensional space $\widehat{\mathfrak{E}}_{N}(h)($ see (6.14)) and it follows:

$$
\hat{\lambda}_{N}(h) \geq \nu\left(\alpha_{0}, \eta_{0}\right)+\mu_{2, n} h^{1 / 2}+o\left(h^{1 / 2}\right) .
$$

This is exactly the rescaled statement of Proposition 6.1.

- Acknowledgements. The authors would like to thank the Mittag-Leffler Institute where the final ideas of this paper were discussed. The authors are also grateful to V. Bonnaillie-Noël and M. Dauge for helpful discussions and their interest in this work.

\section{REFERENCES}

[1] S. AGmon. Lectures on exponential decay of solutions of second-order elliptic equations: bounds on eigenfunctions of $N$-body Schrödinger operators, volume 29 of Mathematical Notes. Princeton University Press, Princeton, NJ 1982.

[2] P. Bauman, D. Phillips, Q. TANG. Stable nucleation for the Ginzburg-Landau system with an applied magnetic field. Arch. Rational Mech. Anal. 142(1) (1998) 1-43.

[3] A. BernofF, P. SternBerG. Onset of superconductivity in decreasing fields for general domains. J. Math. Phys. 39(3) (1998) 1272-1284.

[4] C. Bolley, B. HelfFer. The Ginzburg-Landau equations in a semi-infinite superconducting film in the large $\kappa$ limit. European J. Appl. Math. 8(4) (1997) 347-367.

[5] V. BonNAILLIE. On the fundamental state energy for a Schrödinger operator with magnetic field in domains with corners. Asymptot. Anal. 41(3-4) (2005) 215-258.

[6] V. Bonnaillie-NoËL. Harmonic oscillators with Neumann condition of the half-line. Commun. Pure Appl. Anal. 11(6) (2012) 2221-2237.

[7] V. Bonnaillie-NoËL, M. DAUGE. Asymptotics for the low-lying eigenstates of the Schrödinger operator with magnetic field near corners. Ann. Henri Poincaré 7(5) (2006) 899-931.

[8] V. Bonnaillie-NoËl, M. Dauge, N. Popoff, N. Raymond. Discrete spectrum of a model Schrödinger operator on the half-plane with Neumann conditions. ZAMP 63(2) (2012) 203-231.

[9] L. Charles, S. VŨ NGọC. Spectral asymptotics via the semiclassical Birkhoff normal form. Duke Math. $J .143(3)$ (2008) 463-511.

[10] J.-M. Combes, P. Duclos, R. SeILER. The Born-Oppenheimer approximation. Rigorous atomic and molecular physics (eds G. Velo, A. Wightman). (1981) 185-212.

[11] H. L. CyCON, R. G. Froese, W. KiRSCH, B. Simon. Schrödinger operators with application to quantum mechanics and global geometry. Texts and Monographs in Physics. Springer-Verlag, Berlin, study edition 1987.

[12] M. Del Pino, P. L. Felmer, P. Sternberg. Boundary concentration for eigenvalue problems related to the onset of superconductivity. Comm. Math. Phys. 210(2) (2000) 413-446.

[13] M. Dimassi, J. SJöstrand. Spectral asymptotics in the semi-classical limit, volume 268 of London Mathematical Society Lecture Note Series. Cambridge University Press, Cambridge 1999.

[14] N. DOMBROWSKI, N. RAYMOND. Semiclassical analysis with vanishing magnetic fields. To appear in JST (2012). 
[15] J. V. Egorov. Canonical transformations and pseudodifferential operators. Trudy Moskov. Mat. Obšč. 24 (1971) 3-28.

[16] S. Fournais, B. HelfFer. Accurate eigenvalue asymptotics for the magnetic Neumann Laplacian. Ann. Inst. Fourier (Grenoble) 56(1) (2006) 1-67.

[17] S. FournaIS, B. HelfFER. Spectral methods in surface superconductivity. Progress in Nonlinear Differential Equations and their Applications, 77. Birkhäuser Boston Inc., Boston, MA 2010.

[18] P. GRISVARD. Smoothness of the solution of a monotonic boundary value problem for a second order elliptic equation in a general convex domain. In Ordinary and partial differential equations (Proc. Fourth Conf., Univ. Dundee, Dundee, 1976), pages 135-151. Lecture Notes in Math., Vol. 5641976.

[19] P. GRISVARD. Elliptic problems in nonsmooth domains, volume 24 of Monographs and Studies in Mathematics. Pitman (Advanced Publishing Program), Boston, MA 1985.

[20] V. V. GRUŠIN. Hypoelliptic differential equations and pseudodifferential operators with operator-valued symbols. Mat. Sb. (N.S.) 88(130) (1972) 504-521.

[21] B. HELFFER. Semi-classical analysis for the Schrödinger operator and applications, volume 1336 of Lecture Notes in Mathematics. Springer-Verlag, Berlin 1988.

[22] B. HelfFer, Y. A. Kordyukov. Semiclassical spectral asymptotics for a two-dimensional magnetic Schrödinger operator: the case of discrete wells. In Spectral theory and geometric analysis, volume 535 of Contemp. Math., pages 55-78. Amer. Math. Soc., Providence, RI 2011.

[23] B. HelfFer, A. Morame. Magnetic bottles in connection with superconductivity. J. Funct. Anal. 185(2) (2001) 604-680.

[24] B. Helffer, A. Morame. Magnetic bottles for the Neumann problem: the case of dimension 3. Proc. Indian Acad. Sci. Math. Sci. 112(1) (2002) 71-84. Spectral and inverse spectral theory (Goa, 2000).

[25] B. Helffer, A. Morame. Magnetic bottles for the Neumann problem: curvature effects in the case of dimension 3 (general case). Ann. Sci. École Norm. Sup. (4) 37(1) (2004) 105-170.

[26] L. HÖRMANDER. The analysis of linear partial differential operators. III. Classics in Mathematics. Springer, Berlin 2007. Pseudo-differential operators, Reprint of the 1994 edition.

[27] M. ISMAIL, R. ZhANG. On the Hellmann-Feynman theorem and the variation of zeros of certain special functions. Adv. in Appl. Math. 9(4) (1988) 439-446.

[28] K. LU, X.-B. PAN. Eigenvalue problems of Ginzburg-Landau operator in bounded domains. J. Math. Phys. 40(6) (1999) 2647-2670.

[29] K. LU, X.-B. PAN. Surface nucleation of superconductivity in 3-dimensions. J. Differential Equations 168(2) (2000) 386-452. Special issue in celebration of Jack K. Hale's 70th birthday, Part 2 (Atlanta, GA/Lisbon, 1998).

[30] A. Martinez. Développements asymptotiques et effet tunnel dans l'approximation de Born-Oppenheimer. Ann. Inst. H. Poincaré Phys. Théor. 50(3) (1989) 239-257.

[31] A. MARTINEZ. An introduction to semiclassical and microlocal analysis. Universitext. Springer-Verlag, New York 2002.

[32] X.-B. Pan. Upper critical field for superconductors with edges and corners. Calc. Var. Partial Differential Equations 14(4) (2002) 447-482.

[33] N. Popoff. Sur l'opérateur de Schrödinger magnétique dans un domaine diédral. (thèse de doctorat). Université de Rennes 1 (2012).

[34] N. RAYMOND. Sharp asymptotics for the Neumann Laplacian with variable magnetic field: case of dimension 2. Ann. Henri Poincaré 10(1) (2009) 95-122.

[35] N. RAYMOND. On the semiclassical 3D Neumann Laplacian with variable magnetic field. Asymptot. Anal. 68(1-2) (2010) 1-40.

[36] N. RAYMOND. From the Laplacian with variable magnetic field to the electric Laplacian in the semiclassical limit. To appear in APDE (2012).

[37] N. RAYMOND. Semiclassical 3D Neumann Laplacian with variable magnetic field : a toy model. Comm. in PDE 37(9) (2012) 1528-1552.

[38] D. RoberT. Autour de l'approximation semi-classique, volume 68 of Progress in Mathematics. Birkhäuser Boston Inc., Boston, MA 1987. 
[39] S. VŨ NGọc. Systèmes intégrables semi-classiques: du local au global, volume 22 of Panoramas et Synthèses [Panoramas and Syntheses]. Société Mathématique de France, Paris 2006.

[40] S. VŨ NGọc. Quantum Birkhoff normal forms and semiclassical analysis. In Noncommutativity and singularities, volume 55 of Adv. Stud. Pure Math., pages 99-116. Math. Soc. Japan, Tokyo 2009.

IRMAR (UMR 6625), Université de Rennes 1, Campus de Beaulieu, 35042 Rennes Cedex (FRANCE) 